\title{
Characterization of cold shock domain proteins and SUMOylation system from Oryza sativa
}

Vijaya Vardhana Reddy Chaikam

West Virginia University

Follow this and additional works at: https://researchrepository.wvu.edu/etd

\section{Recommended Citation}

Chaikam, Vijaya Vardhana Reddy, "Characterization of cold shock domain proteins and SUMOylation system from Oryza sativa" (2009). Graduate Theses, Dissertations, and Problem Reports. 4448.

https://researchrepository.wvu.edu/etd/4448

This Dissertation is protected by copyright and/or related rights. It has been brought to you by the The Research Repository @ WVU with permission from the rights-holder(s). You are free to use this Dissertation in any way that is permitted by the copyright and related rights legislation that applies to your use. For other uses you must obtain permission from the rights-holder(s) directly, unless additional rights are indicated by a Creative Commons license in the record and/ or on the work itself. This Dissertation has been accepted for inclusion in WVU Graduate Theses, Dissertations, and Problem Reports collection by an authorized administrator of The Research Repository @ WVU.

For more information, please contact researchrepository@mail.wvu.edu. 


\title{
Characterization of cold shock domain proteins and SUMOylation system from Oryza sativa
}

\author{
Vijaya Vardhana Reddy Chaikam
}

\author{
Dissertation submitted to the \\ Davis College of Agriculture, Forestry and Consumer Sciences \\ at West Virginia University \\ in partial fulfillment of the requirements \\ for the degree of
}

Doctor of Philosophy

in

Genetics and Developmental Biology

Dale Karlson, Ph.D., Chair

Daniel Panaccione, Ph.D.

Carina Barth, Ph.D.

Jed Doelling, Ph.D.

Todd West, Ph.D.

Division of Plant and Soil Sciences,

Morgantown, WV

2009

Keywords: cold acclimation, gene expression, development, SUMO, protein localization, stress responses, SUMO conjugates 


\section{$\underline{\text { ABSTRACT }}$ \\ Characterization of cold shock domain proteins and SUMOylation system from Oryza sativa}

\section{Vijaya Vardhana Reddy Chaikam}

More than half of the world's population depends on rice (Oryza sativa) for their nutritional requirements. Rice is the most important commercially grown agricultural plant and is also rapidly becoming an important scientific model system for monocots. With the availability of its complete genome sequence, identification and functional characterization of rice genes is gaining momentum. Knowledge resulting from these studies about the functions of genes in rice could lead to enhanced agronomic traits such as increased yield, biotic and abiotic stress tolerance. In this study, I aimed to understand the role of cold shock domain(CSD) proteins, SUMOylation system and their interaction in rice.

In the first part of this study, two novel cold shock domain proteins from rice (OsCSP) were cloned and subsequently characterized their roles during stress conditions and development. OsCSP1 and OsCSP2 (Oryza sativa CSD protein) encode putative proteins consisting of an $\mathrm{N}$-terminal CSD and glycine-rich regions that are interspersed by 4 and $2 \mathrm{CX}_{2} \mathrm{CX}_{4} \mathrm{HX}_{4} \mathrm{C}(\mathrm{CCHC})$ retroviral-like zinc fingers, respectively. Using an in vitro DNA binding assay, I demonstrate that OsCSPs exhibit conserved ssDNA binding activity. In vivo functional complementation in a cold-sensitive bacterial strain, that lacks four cold inducible cold shock domain proteins revealed that OsCSPs function as RNA chaperones, similar to their bacterial and winter wheat counterparts. To understand the functions of these genes in rice, I studied the transcriptional regulation in response to abiotic stress conditions. Under cold stress, OsCSP transcript levels are only transiently and marginally increased and the encoded proteins did not accumulate. These transcript and protein data are in sharp contrast with the observed data for winter wheat and Arabidopsis cold shock domain proteins under cold stress. In these species, both transcripts and protein levels of CSPs are increased upon cold stress. Based on these data, it can be hypothesized that the accumulation of cold shock domain proteins may play an important role in determining the cold acclimation capability of the plants. Expression analysis at the protein and RNA levels during development revealed that OsCSPs are highly expressed in the reproductive and meristematic tissues. These results indicate a potential role for rice cold shock domain proteins in plant growth and reproductive development.

In this study, I also characterized the post-translational modification of plant cold shock domain proteins by SUMOylation. Post-translational modifications can impart rapid changes in protein function.. SUMOylation involves the reversible attachment of a small protein called SUMO (small ubiquitin-like modifier) to target proteins. The SUMO protein has a similar three dimensional structure as that of ubiquitin and the process of 
SUMOylation is very similar to that of ubiquitination. However, unlike ubiquitination, SUMOylation is not implicated in protein degradation. SUMO modification can affect the target protein stability, sub-cellular localization protein-protein interactions. Using a computational approach on rice and Arabidopsis cold shock domain proteins, I identified canonical SUMOylation motifs in both rice CSPs and one of the Arabidopsis CSPs. Using in vitro assays, I demonstrated that both OsCSPs can undergo SUMOylation. Using mutational approaches, I identified an important lysine residue for SUMOylation in Arabidopsis AtCSP1. By employing GFP-tagged wild-type and variant AtCSP1 proteins, I demonstrate that SUMOylation appears to affect AtCSP1 protein localization.

In another study, I characterized the entire SUMO conjugation system in rice. The process of SUMOylation involves a cascade of enzymatic reactions involving activation (E1) enzymes, conjugation (E2) enzymes and ligation (E3) enzymes. I compared the protein sequences of all these genes from rice with those from Arabidopsis, yeast and human. This revealed a high amino acid sequence conservation of individual SUMOylation components from yeast to plants and animals. In Arabidopsis, the SUMOylation system has been implicated in plant development and in mediating abiotic stress responses. To understand the role of the rice SUMOylation system during development, I studied the SUMO conjugate profiles and the expression of individual SUMO component genes in various tissues at different stages of plant development. The highest levels of SUMOylated proteins were observed in panicles and meristematic tissues. Expression studies revealed that SUMO component genes are highly expressed in reproductive tissues like developing seeds and panicles. Together, these data implicate an important role for the rice SUMOylation system in plant growth and reproductive development. To understand the role of SUMOylation system in rice, I studied SUMO conjugate profiles and the transcriptional regulation of individual SUMO components during cold, salt and ABA stress conditions. Rice responds to these stresses by accumulating SUMO conjugated proteins, suggesting that protein SUMOylation helps to mediate plant stress responses. Studies on the transcriptional regulation of individual SUMO pathway genes during these stress conditions revealed that most are transcriptionally down-regulated. However, a particular SUMO E3 ligase gene (OsSIZ2) is transiently up-regulated upon exposure to all three stress conditions. Considering the importance of E3 ligases in improving the efficiency and specificity of the SUMO conjugation reactions, OsSIZ2 may mediate accumulation of SUMO conjugates during these stress conditions. Taken together, these data suggest a role for SUMOylation in rice development and stress responses. 


\title{
Dedicated to
}

\author{
My late father Rama Yogi Reddy \\ And \\ Mother Prabhavathi
}




\section{Acknowledgements}

I acknowledge the support, guidance and encouragement given by Dr. Dale Karlson, chair of my advisory committee. My association with Dr. Karlson not only helped me to grow as a scientist, it also influenced all aspects of my life very positively. I truly cherish all the moments in his lab and cannot find words to explain my gratitude for all the good he brought into my life. I also thank my committee members Dr. Daniel Panaccione, Dr. Jed Doelling, Dr. Carina Barth and Dr. Todd West for their guidance towards my course work and research. I am also grateful for their suggestions and help in my job search. I thank my labmates Dr. Kentaro Nakaminami, Yongil Yang, Kari Thompson, Swamy Tripurani and Prashanthi Mulinti for creating a wonderful lab environment. They are all very helpful, kind and friendly. The time I spent with them includes many happiest and unforgettable moments of my life. I thank Swamy and Prashanthi for the care and affection they have shown on me and for making a homely environment in the lab.

I thank Dr. Barton Baker for accepting me into the program and supporting me financially. I remember and acknowledge the help rendered by all the green house staff in growing rice plants. I thank all the students of Genetics and Developmental biology for creating an excellent intellectual environment. I thank Dr. Jianbo Yao from Animal Science Division for his good will and valuable suggestions.

I cherish all the moments I spent with my friend Brahma Reddy. I thank him for all the support, affection and care he has shown on me. I also thank Madhu, Raghu and Venky for making my life more enjoyable in Morgantown. I thank my fiancée Jyothsna for her love, support, encouragement and patience.

The dreams, expectations and good will of my mother, sisters (Usha and Vasavi) and brother-in laws always kept me motivated. I remember my late father for his encouragement and care. Nothing can replace the love and affection of my family and I have no words to expresses gratitude for them. 


\section{Table of contents}

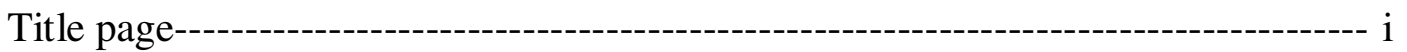

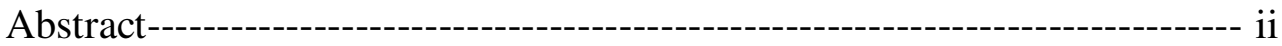

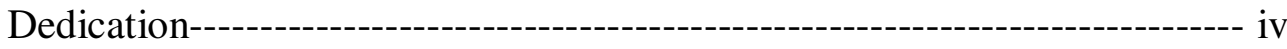

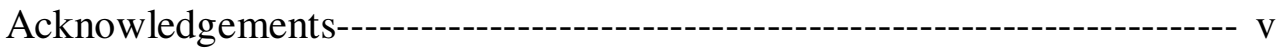

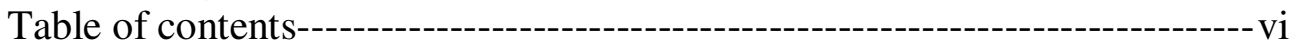

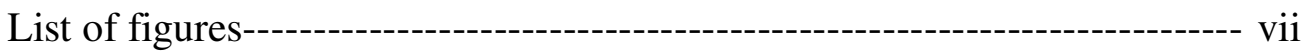

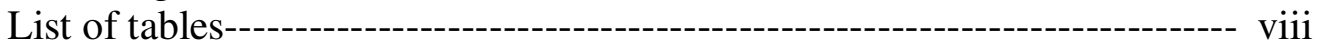

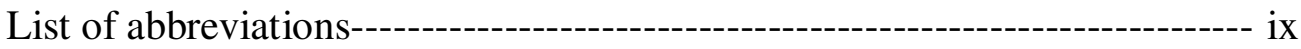

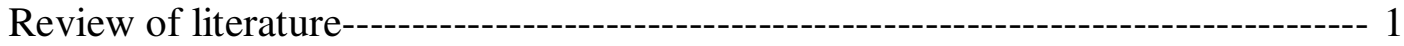

Cold shock domain proteins---------------------------------------------- 1

SUMOylation system------------------------------------------------- 11

Chapter 1: Characterization of cold shock domain proteins from rice-------------- 23

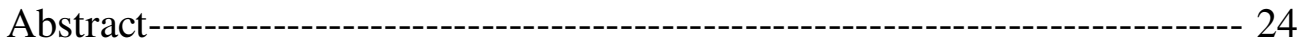

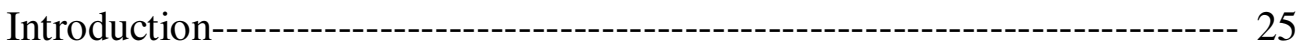

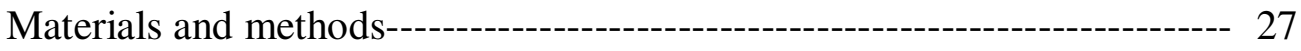

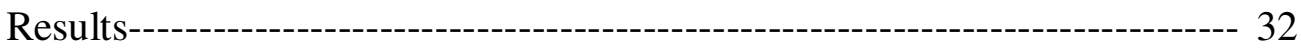

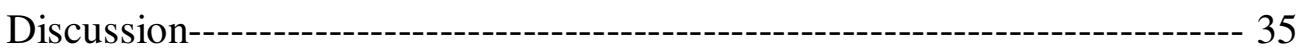

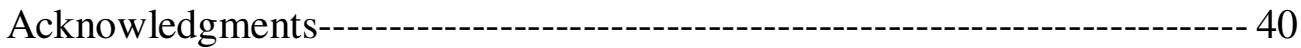

Figures------------------------------------------------------------------------- 41

Chapter 2: SUMOylation of plant cold shock domain proteins------------- 50

Abstract-----------o- 51

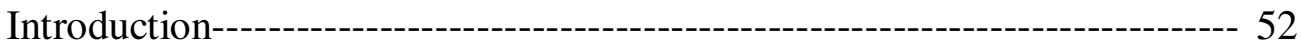

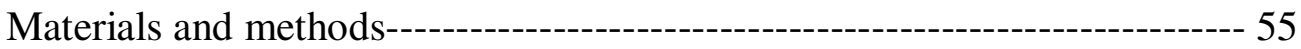

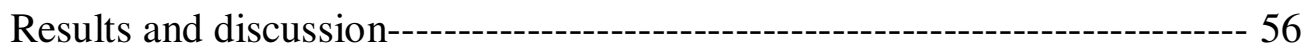

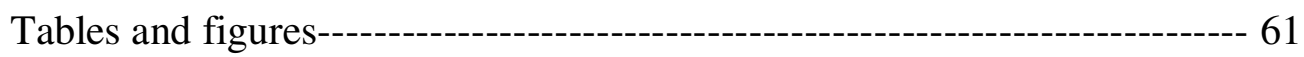

Chapter 3: SUMOylation system of rice--------_- 65

Abstract-------o- 66

Introduction--- 67

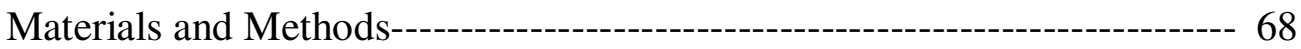

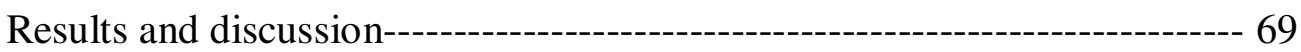

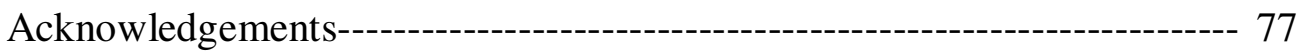

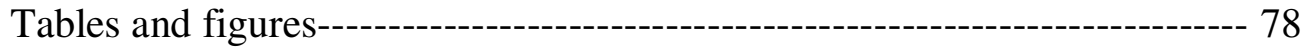

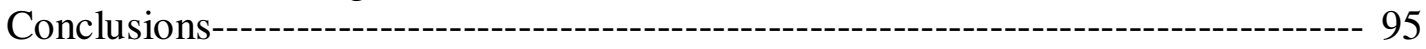

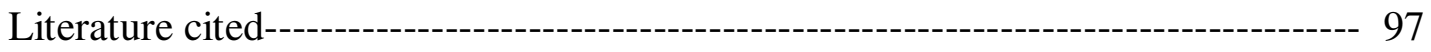




\section{List of figures}

\section{Chapter-1:}

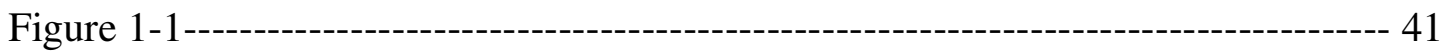

Figure 1-2---------------------------------------------------------------------------------------------- 42

Figure 1-3--------------------------------------------------------------------------------------------- 43

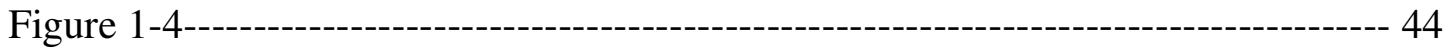

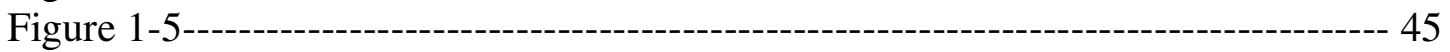

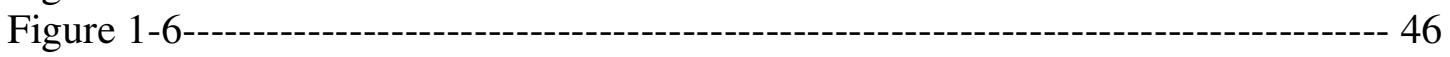

Figure 1-7--------------------------------------------------------------------------------------------- 47

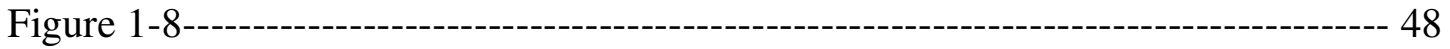

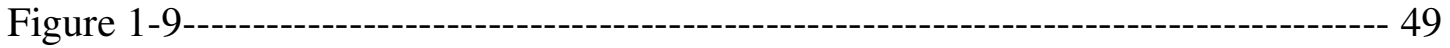

Chapter-2:

Figure 2-1---------------------------------------------------------------------------------------------- 62

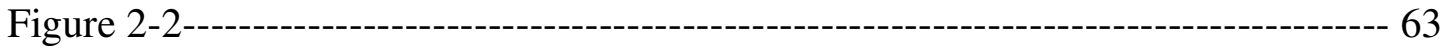

Figure 2-3------------------------------------------------------------------------------------------- 64

Chapter-3:

Figure 3-1-------------------------------------------------------------------------------------------- 80

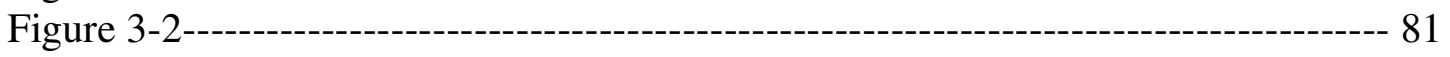

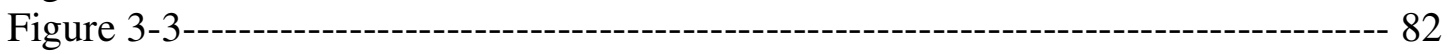

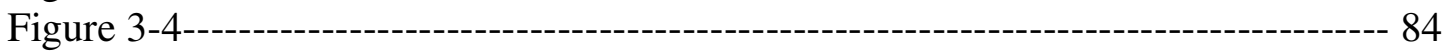

Figure 3-5------------------------------------------------------------------------------------------ 85

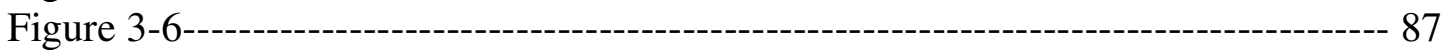

Figure 3-7-------------------------------------------------------------------------------------- 89

Figure 3-8------------------------------------------------------------------------------------------- 91

Figure 3-9------------------------------------------------------------------------------------------ 93 


\section{$\underline{\text { List of tables }}$}

Table 2-1--------------------------------------------------------------------------------------------- 61

Table 3-1-------------------------------------------------------------------------------------------- 78

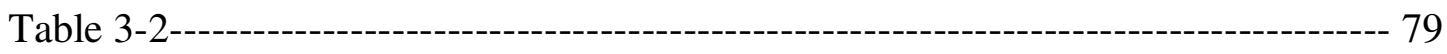




\section{$\underline{\text { List of abbreviations }}$}

$\begin{array}{ll}\text { CSD } & \text { Cold Shock Domain } \\ \text { CSP/Csp } & \text { Cold Shock Protein } \\ \text { OsCSP } & \text { Oryza sativa Cold Shock Protein } \\ \text { AtCSP } & \text { Arabidopsis thaliana Cold Shock Protein } \\ \text { WCSP1 } & \text { Wheat Cold Shock Protein1 } \\ \text { YB-1 } & \text { Y-box binding protein1 } \\ \text { SUMO } & \text { Small Ubiquitin like Modifier } \\ \text { SAE } & \text { SUMO Activation Enzyme } \\ \text { SCE } & \text { SUMO Conjugation Enzyme } \\ \text { PIAS } & \text { Protein Inhibitor of Activated STAT } \\ \text { PC2 } & \text { Poly Comb2 } \\ \text { RAN-BP2 } & \text { Ran-binding protein2 } \\ \text { NSE2/MMS1 } & \text { non-SMC element/Methyl methanosulfonate sensitive1 } \\ \text { ABA } & \text { Abscisic acid }\end{array}$




\section{REVIEW OF LITERATURE}

\section{Cold shock domain proteins}

The cold shock domain (CSD) is among the most ancient and well conserved nucleic acid binding domains distributed within bacteria, animals and plants. The CSD facilitates binding to RNA, ssDNA and dsDNA, and most functions attributed to cold shock domain proteins (CSPs) are mediated by this nucleic acid binding activity. In prokaryotes, cold shock domain proteins are comprised of a single CSD only and are referred as cold shock proteins (Csps). In higher mammalian model systems, various auxiliary domains are present in addition to the CSD and are commonly named as Y-Box binding (YB) proteins. Similar to animal CSPs, plant CSPs contain auxiliary C-terminal domains in addition to their N-terminal CSD. In this review, the structure, function and regulation of plant CSPs are compared and contrasted to the characteristics of bacterial and animal CSPs.

\section{Cold shock domain proteins structure}

Bacteria encode Cspsthat are of small size (67-73 amino acids) and consist of a single nucleic acid-binding CSD (Goldstein et al., 1990; Graumann and Marahiel, 1996). Within the CSD, two consensus RNA binding motifs are present (RNP-1 and RNP-2), which are also present in RRM-type RNA binding proteins (Landsman, 1992; Schindelin et al., 1993; Manival et al., 2001). Studies on the three-dimensional structures of two $E$. coli Csps (CspA and CspB) placed the RNP-1 and RNP-2 motifs on separate, juxtaposed, adjacent B-strands within the CSD (Newkirk et al., 1994; Schindelin et al., 1994; Feng et al., 1998). A similar structure was also observed for the CspB protein from Bacillus subtilis (Schindelin et al., 1993). Aromatic residues residing within the RNP-1 and RNP2 motifs are critical for facilitating ssDNA-binding activity by enabling base stacking (Schindelin et al., 1994; Schroder et al., 1995) without apparent sequence specificity. CspB from Bacillus subtilis and CspD from E. coli purify as dimers in solution. In the case of B. subtilis $\mathrm{CspB}$, dimers are formed between two anti-parallel CspB molecules through interactions between the $\beta 4-\beta 4$ and $\beta 4-\mathrm{N}$ terminus (Schindelin et al., 1993; 
Schnuchel et al., 1993). On the other hand, CspA from E. coli was crystallized as a monomer (Newkirk et al., 1994; Schindelin et al., 1994).

Unlike bacterial Csps, their eukaryotic counterparts contain auxiliary domains in addition to a cold shock domain. In the human CSP, YB-1, three structural domains can be recognized: a small domain at the N-terminus which is Ala and Pro rich (A/P domain), a central cold shock domain (CSD), and a C-terminal domain (C domain) with alternating clusters of positively and negatively charged amino acid residues (four clusters of each charge) (Matsumoto and Wolffe, 1998; Bader and Vogt, 2005). Among bacterial Csps and YB-1, the $\sim 70$ residues that comprise the CSD represent the only region that exhibits a high level of sequence conservation. Studies on the CSD of YB-1 revealed a $\beta$-barrel spatial structure bearing similarity to bacterial Csps with a similar arrangement of RNA binding motifs (Schindelin et al., 1993; Schnuchel et al., 1993; Kloks et al., 2002). The C-terminal auxiliary domain was proposed to have a nonspecific affinity for RNA and DNA which may result from its interaction with negatively charged phosphate groups of nucleic acids (Evdokimova and Ovchinnikov, 1999). It also serves as a docking site for other interacting proteins (Wolffe, 1994). Similar to bacterial Csps, YB-1 forms large homomultimeric complexes (Evdokimova et al., 1995; Gaudreault et al., 2004). The domain structure among vertebrate Y-box proteins is essentially similar with 100\% sequence homology in the CSD. The C-terminal tail domain in vertebrate Y-box proteins is highly divergent and is used to distinguish germ cell and somatic cell type Y-box proteins (Skabkin et al., 2006). The three dimensional structure of a plant CSD has not yet been reported. Plants CSPs are glycine-rich proteins and are distinct in that they contain two types of nucleic acid-binding modules, a single N-terminal CSD and variable numbers of $\mathrm{C}$-terminal retroviral-like $\mathrm{CCHC}$ zinc fingers that are interspersed by glycinerich regions (Karlson and Imai, 2003).

\section{Functions of Cold Shock Domain proteins}

\section{Functions in cold stress adaptation}

Bacterial Csps play critical roles during the cold adaptation process. E. coli contains nine Csps named from CspA to CspI (Yamanaka et al., 1998), of which only CspA, CspB, CspE, CspG, and CspI are cold inducible (Goldstein et al., 1990; Lee et al., 1994; 
Nakashima et al., 1996; Wang et al., 1999). Upon cold stress, synthesis of the major cold shock domain protein CspA is very highly induced, it accumulates to comprise more than $10 \%$ of the total protein in cold-shocked cells (Goldstein et al., 1990). The binding of CspA to RNA is associated with the destabilization of the secondary structures in RNA, thereby facilitating translation at low temperatures, where formation of secondary structures in RNA is common (Jiang et al., 1997). Later, an RNA chaperone activity was attributed to CspE protein (Phadtare et al., 2002; Phadtare and Severinov, 2005). However, a quadruple deletion of $\mathrm{CspA}, \mathrm{CspB}, \mathrm{CspG}$, and $\mathrm{CspE}$ is required to make the E. coli cells cold sensitive (Xia et al., 2001) indicating the redundancy of function for Csps during exposure to low temperature stress.

Until recently, the cellular functions of vertebrate Y-box proteins were not correlated to low temperature stress. Recently, a downshift in the cell culture temperature of YB-1-depleted chicken cells was shown to lead to growth arrest. Cell growth under low temperature was restored by the expression of an epitope-tagged YB-1 protein in the gene disruptants. This was the first indication that CSD proteins are important for cold adaptation in higher vertebrates (Matsumoto et al., 2005).

In plants, expression studies on a wheat cold shock domain protein (WCSP1) revealed an up-regulation on both the transcript and protein level during cold acclimation (Karlson et al., 2002). The WCSP1 protein complements the cold sensitive phenotype of the quadruple Csp-deletion strain of E. coli (BX04), indicating that this protein can function in vivo in a bacterial system. Similar to some bacterial Csps, WCSP1 can also function as a transcription anti-terminator indicating a role in transcription (Nakaminami et al., 2006). Expression studies revealed that some Arabidopsis CSP transcripts are also increased upon cold stress treatment, indicating a potential role in cold adaptation (Karlson and Imai, 2003; Kim et al., 2007; Sasaki et al., 2007). Very recently, Arabidopsis thaliana old shock domain proteins (AtCSPs) were shown to promote cold adaptation in bacteria (Kim et al., 2007; Sasaki et al., 2007). Another recent study in rice, a plant which cannot acclimate to cold conditions, revealed that the expression of cold shock domain proteins was not induced in response to low temperature stress. However, in vitro and in vivo studies demonstrated that these proteins were capable of functioning in a similar fashion as wheat and Arabidopsis cold shock domain proteins, indicating a 
correlative role for cold shock domain proteins in the cold acclimation process (Chaikam and Karlson, 2008). Despite this evidence about the role of cold shock domain proteins in cold adaptation, their mode of action in planta and their importance during cold acclimation are poorly understood.

\section{Functions in DNA metabolism}

In E. coli, camphor induced chromosome decondensation was reversed by overexpression of CspE and CspC (Hu et al., 1996). In addition, CspD was shown to act as a novel inhibitor of DNA replication and play a role in chromosome replication during nutrient stress (Yamanaka and Inouye, 2001a). Hence, bacterial Csps are implicated in maintaining chromosome structure and DNA replication.

Similar to prokaryotic Csps, the higher eukaryotic cold shock domain protein, YB-1, plays an important role in various aspects of DNA metabolism. YB-1 moves from the cytoplasm to the nucleus in response to UV light and DNA-damaging chemicals (Koike et al., 1997; Gaudreault et al., 2004). The YB-1 protein exhibits a stronger affinity toward secondary structures in damaged DNA and aids in DNA repair (Ise et al., 1999; Gaudreault et al., 2004). YB-1 plays a role in DNA recombination by promoting complementary DNA strand exchange (Ise et al., 1999; Skabkin et al., 2001). Similar to bacterial Csps, YB-1 functions in DNA replication (Levenson et al., 2000; En-Nia et al., 2005). Thus, YB-1 appears to be involved in majority of the DNA dependent process. To date, plant CSPs have not been studied in relation to DNA recombination and repair.

\section{Transcription}

In E. coli, Csps affect transcription by acting as transcription antiterminators. In in vitro studies, CspE protein inhibited phage lambda Q-mediated transcriptional antitermination (Hanna and Liu, 1998). Over expression of the CspE protein resulted in high expression of several promoter-distal genes in the metY-rpsO operon due to transcription antitermination (Bae et al., 2000) Using in vitro and in vivo studies, CspA, CspE, and $\mathrm{CspC}$ proteins were demonstrated to work as transcription antiterminators at $\rho$ independent terminators (Bae et al., 2000). 
In addition, CspE associates with nascent RNA in transcription elongation complexes implicating a role in transcription (Hanna and Liu, 1998). The transcription pause efficiency of RNA polymerase was shown to be increased by CspE and CspA protein (Jiang et al., 1996b; Bae et al., 1999).

The role of human YB-1 has been well studied in relation to transcription. Initially, this protein was identified by its ability to bind to the Y-box sequence (inverted CCAAT motifs) of MHC class II promoters (Didier et al., 1988). The binding of YB-1 to Y-box sequences influences the transcription of genes either positively or negatively by binding (Swamynathan et al., 1998; Kohno et al., 2003). Numerous genes that are important for normal cellular functions are transcriptionally regulated by the YB-1 protein (Skabkin et al., 2006). During the G1/S phase transition or in response to thrombin, YB-1 is transferred from the cytoplasm to the nucleus and activates the transcription of certain genes like cyclin A and B1 (Jurchott et al., 2003) or those involved with endothelial cell differentiation (Stenina et al., 2001). In the case of plant CSPs, no evidence has been produced to suggest that they may function as transcription factors. However, wheat WCSP1 and Arabidopsis AtCSP2 (AtGRP2) are localized to the nucleus (Nakaminami et al., 2006; Fusaro et al., 2007; Sasaki et al., 2007), suggesting a possible role in transcription.

\section{Translation}

The majority of E. coli Csps are capable of binding RNA (Jiang et al., 1997; Yamanaka et al., 1998; Phadtare and Inouye, 1999). During exposure to low temperature stress conditions, stabilized secondary structure formation in RNA is thermodynamically favored and hinders the translation of transcripts. It was shown experimentally in $E$. coli that the major cold-induced Csp ( $\mathrm{CspA}$ ) acts as an RNA chaperone and destabilizes secondary structures (Jiang et al., 1997). CspE was later shown to melt partially double stranded and hairpin structures (Phadtare and Severinov, 2005). The CspE protein was also shown to by bind poly-A tails and thereby stabilize mRNA by reducing degradation by PNPase and RNaseE (Feng et al., 2001). Transcripts of the global stress response regulator, rpoS and stress response protein, uspA, were shown to be stabilized by $\mathrm{CspC}$ 
and CspE (Phadtare and Inouye, 2001). Hence, bacterial Csps are believed to function in translation by acting as chaperones and preventing mRNA degradation.

The role of YB-1 in translation has been extensively studied. YB-1 protein, known originally as p50, was detected in mRNP (messenger ribonucleoprotein) preparations from various cells and organisms (Preobrazhensky and Spirin, 1978; Dreyfuss, 1986). YB-1 exhibits a high affinity for mRNPs and acts as a structural protein in their spatial organization (Minich et al., 1993; Bader and Vogt, 2005). YB-1 exists as a major component of free mRNPs that are not currently being translated, while the polysomal mRNPs, which are being translated, contain poly(A)-binding protein (PABP) along with YB-1 (Blobel, 1973; van Venrooij et al., 1977; Minich and Ovchinnikov, 1992)

YB-1 accompanies mRNAs throughout their "life cycle" by binding to newly emerging pre-mRNA on chromosomes (Kohno et al., 2003; Soop et al., 2003). Depending on the ratio of YB-1 to mRNA, YB-1 differentially affects translation. Translation is stimulated when there is little YB-1, and completely suppressed at a higher YB-1/mRNA ratio (Minich and Ovchinnikov, 1992). The YB-1 mediated regulation of translation occurs only during translation initiation (Evdokimova et al., 1998; Nekrasov et al., 2003). It was proposed thatYB1 stimulates translation initiation by promoting the 43S preinitiation complex to scan the 5'-untranslated region (5'-UTR) in search for the initiation codon (Evdokimova et al., 1998). YB-1 inhibits translation by blocking the interaction between mRNA and translation initiation factors, primarily eIF4G, at the first step of initiation (Nekrasov et al., 2003). 5'-capped mRNAs were found to be stabilized by YB-1 in both in vitro and in vivo studies (Chen et al., 2000; Evdokimova et al., 2001; Nekrasov et al., 2003). The cold shock domain of YB-1 interacts with the cap, and this interaction inhibits the action of cap-cleaving enzymes. Thus YB-1 promotes the accumulation of mRNAs in the form of free mRNPs for stable storage by suppressing translation and the removal of the 5' cap (Evdokimova et al., 2001; Nekrasov et al., 2003; Skabkin et al., 2006). In addition to a role in translation initiation, evidence is accumulating that YB-1 plays a role in alternative splicing of pre-mRNA in the cell nucleus (Chansky et al., 2001; Stickeler et al., 2001). 
In plants, the role of cold shock domain proteins in the translation process has not been studied. Plant CSPs can complement the cold-sensitive phenotype in the E. coli CSP quadruple deletion strain (BX04), suggesting that plant CSPs can also act as chaperones during cold stress (Nakaminami et al., 2006; Kim et al., 2007). The subcellular localization of WCSP1 to the endoplasmic reticulum suggests that it may perform a role in translation (Nakaminami et al., 2006). NAB1, a CSP from Chlamydomonas was shown to stabilize the mRNA of the chlorophyll binding protein mRNA and repress its translation by sequestering it into non-translated mRNPs at the translation pre-initiation stage (Mussgnug et al., 2005). Taken together, these results suggest a potential role for plant CSPs in translation.

\section{Regulation of expression of cold shock domain genes}

Among the bacterial Csps, E. coli CspA is the best studied in terms of gene expression. The previous notion that CspA is regulated solely by a cold responsive transcription factor (Tanabe et al., 1992) was proven to be incorrect with the discovery that the natural promoter of CspA is not required for its cold induction (Brandi et al., 1996; Fang et al., 1997). The $\operatorname{Csp} A$ transcript is highly unstable at $37^{\circ} \mathrm{C}$ and its stability is greatly increased after cold shock (Goldenberg et al., 1996; Fang et al., 1997). Subsequent experiments revealed that the 5' untranslated region (UTR) of CspA transcripts play a critical role in conferring the instability of the transcript (Brandi et al., 1996). The complete deletion of the 5'UTR - resulted in high levels of CspA transcripts at $37^{\circ} \mathrm{C}$ (Fang et al., 1997). The discovery of potential RNaseE recognition elements in the 5' UTR led to the hypothesis that $\operatorname{CspA}$ transcript cleavage by RNaseE at $37^{\circ}$ Cresult in its extreme instability (Goldenberg et al., 1996). During cold shock, CspA transcripts are initially stabilized, however their stability is decreased during cold acclimation because of the transcript degradation by the PNPase enzyme (Yamanaka and Inouye, 2001b). In addition, "cold box", a negative cis-element in the 5' UTR of the CspA transcript, can be recognized by the CspE or CspA protein, thereby increasing the transcription pause recognition (Jiang et al., 1996a; Bae et al., 1999). Thus $\operatorname{CspA}$ expression is regulated post-transcriptionally through the 5'UTR. 


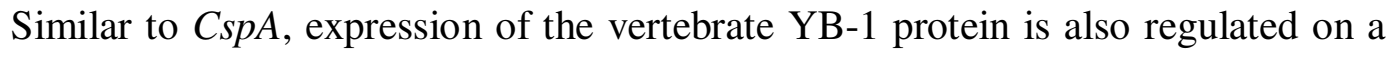
post-transcriptional level. In contrast to CspA, a short nucleotide sequence with in the 3'UTR was found to be important (Skabkina et al., 2003). This short sequence was sufficient to suppress the translation of not only YB-1 mRNA but also of other mRNAs in a cell-free translation system. This observation led to the hypothesis that proteins involved in translation initiation selectively bound to this sequence. Later, UV inducible cross linking experiments identified YB-1 and PABP as the main proteins that bound to this sequence (Skabkina et al., 2003). In a cell free translation system, the YB-1 protein completely suppressed the translation of YB-1 mRNA, while PABP promoted its translation. PABP and YB-1 compete with each other to bind YB-1 mRNA. Specific binding sites of YB-1 and PABP in the 3'UTR were identified using foot-printing (Skabkina et al., 2005). The regions were found to overlap with each other and contain a common octanucleotide motif, UCCA(G/A)CAA. A comparison of the 3'-UTR nucleotide sequences of mRNAs coding for Y-box proteins of various organisms, from $X$. laevis to humans, revealed the evolutionary conservation of the regulatory region, which is enriched in A and C. These studies suggest that most vertebrate cold shock domain genes are regulated post-transcriptionally. The regulatory elements and proteins involved in the regulation of plant CSP gene expression are not known.

\section{Role in development}

The quadruple Csp deletion mutants of $E$. coli showed elongated and filamentous cells at $15^{\circ} \mathrm{C}$ due to defects in cell septum formation (Xia et al., 2001; Kim et al., 2007). The expression of CspD was found to be dramatically induced during stationary phase growth. However, the role of this protein in bacterial development is not clearly understood (Yamanaka and Inouye, 2001a).

In higher eukaryotes, cold shock domain proteins play an important role in development mostly by affecting the translation of certain mRNAs during specific developmental stages. YB-1 plays a role in the stable storage of specific mRNAs by binding to them and repressing their translation until needed. mRNA storage is especially important during certain stages of development, during which cells are rapidly dividing and transcriptional activity is minimal (Sommerville, 1999). For example, the Y-box 
binding protein, FRGY2 is abundant in Xenopus oocytes and is bound to cytoplasmic maternal mRNAs masking their translation (Murray et al., 1991). Similar to the situation observed in Xenopus, Y-box proteins like MSY1, MSY2a, MSY2b, and MSY4, have been identified in the germ cells of mice (Tafuri and Wolffe, 1993; Gu et al., 1998; Davies et al., 2000). MSY2 accounts for $~ 2 \%$ of total oocyte protein and is present in early and mature oocytes but completely disappears at the late two-cell stage embryo stage, suggesting that MSY2 stabilizes and/or regulates the translation of maternal mRNAs (Yu et al., 2001). Reduction of MSY2 levels in mouse oocytes resulted in reduced fertility (Yu et al., 2004). A high abundance of MSY2 is also observed in meiotic and post-meiotic germ cells in testes ( $\mathrm{Gu}$ et al., 1998). It was observed that MSY2 preferentially bound to mRNAs that are transcribed from a Y-box promoter, thereby linking transcription with translational delay in male germ cells. Lack of MSY2 results in spermatogenic arrest and male infertility (Yang et al., 2007). In C. elegans, the Lin28 cold shock domain protein controls developmental transition during early stages (Moss et al., 1997). Collectively, these results suggest a key role for cold shock domain proteins during development by affecting translation.

Using Arabidopsis thaliana as a model plant, CSPs were shown to be important during plant development. When AtGRP2/AtCSP2 expression was up- or down regulated, many developmental abnormalities with respect to flowering time, apical dominance and seed development were observed. Furthermore, the transcripts of this gene are abundant in meristematic areas in which rapid cell divisions occur suggesting this protein may function in mRNA storage (Fusaro et al., 2007). A subsequent extensive study characterized the expression of the entire Arabidopsis CSP family in relation to stages of development and floral and silique development (Nakaminami et al., 2009). In Chlamydomonas, the NAB1 protein was shown to aid in the acclimation to high light conditions by regulating the size of light harvesting antenna of PSII by affecting the mRNA stability of light harvesting chlorophyll binding protein (Mussgnug et al., 2005).

\section{Post-translational modifications of cold shock domain proteins}

Unlike bacterial Csps, several vertebrate cold shock domain proteins have been shown to be modified on the post-translational level. Xenopus FRGY1 and FRGY2 are 
phosphorylated by casein kinase II; this modification promoted their mRNA binding capability, which may affect mRNA silencing during oogenesis (Sommerville and Ladomery, 1996; Matsumoto and Wolffe, 1998). For human YB-1, casein kinase II mediated phosphorylation did not affect its RNA binding ability (Skabkin et al., 2001). Recently, it was discovered that YB-1 can also be phosphorylated by AKT kinase, decreasing its ability of binding to mRNA caps. This affected Cap-dependent translational repression by $\mathrm{YB} 1$, resulting in the activation of silenced mRNA species (Evdokimova et al., 2006). Another vertebrate CSP, PIPPin was shown to be SUMOylated in rat brain in the presence of thyroid but not in its absence in hypothyroidism (Bono et al., 2007). The functional significance of SUMOylation for this protein is not yet understood.

Four Arabidopsis CSPs were shown to undergo phosphorylation in vitro using cell extracts of plants grown under standard conditions (Karlson et al., 2009, Thompson et al. unpublished results). CSPs from various plants were shown to possess one or more SUMOylation signature motifs. Using in vitro assays, AtCSP1 and both rice CSPs were shown to be capable of being SUMOylated.

In summary, CSPs from bacteria and higher vertebrates mediate various cellular processes by binding to nucleic acids. These proteins function in transcription, translation and DNA-dependent processes like recombination and repair. In bacteria, these proteins are critical for survival under cold stress. Even though CSPs are widespread in plants, very little is known about their cellular functions. Expression data for winter wheat and Arabidopsis CSPs suggest that these genes play important roles in plant cold stress responses. To expand our knowledge about plant CSP functions, I initiated functional studies on rice CSPs. 


\section{SUMOylation system}

Protein modification by the addition of chemical groups like phosphate, methyl, acetate, (etc.) or small proteins such as ubiquitin and ubiquitin-like proteins, is a way to rapidly alter the functions of pre-existing proteins; therefore they play critical roles in various cellular processes. Protein modification by ubiquitin conjugation has been studied for many years and its role in target protein degradation via the $26 \mathrm{~S}$ proteasome is well established. SUMO protein, a ubiquitin-like modifier, resembles ubiquitin in its 3-D structure, the presence of a C-terminal diglycine motif, and a similar protein size. However, SUMO only shares $\sim 18 \%$ sequence identity with ubiquitin and possesses a different surface charge topology (Bayer et al., 1998; Mossessova and Lima, 2000; Jin et al., 2001; Muller et al., 2001; Geiss-Friedlander and Melchior, 2007; Zhao, 2007a). It also include an N-terminal unstructured extension of up to 22 residues, not present in ubiquitin, that provides additional interface for protein-protein interactions (Seeler and Dejean, 2003). SUMO has been recently recognized as a common post-translational modifier of proteins that affects protein interactions, localization, and function (Johnson, 2004; Zhao, 2007a).

The majority of identified SUMO targets are nuclear proteins. Therefore, the importance of SUMOylation has been implicated in nuclear functions. However, cytoplasmic and membrane bound proteins have been recently identified as SUMOylation targets indicating a wide range of functions for SUMOylation in different cellular compartments (Seeler and Dejean, 2003; Gill, 2004; Geiss-Friedlander and Melchior, 2007). Many of the SUMOylation target proteins contain a recognizable consensus motif, namely $\psi$-Lys-X-Glu/Asp (where $\psi$ is a large hydrophobic amino acid, most commonly isoleucine or valine, and $\mathrm{X}$ is any residue) (Rodriguez et al., 2001; Sampson et al., 2001). However, some proteins like histones can also undergo SUMOylation even in the absence of this motif (Shiio and Eisenman, 2003; Nathan et al., 2006). In addition to the core $\psi$-Lys-X-Glu/Asp motif, some surrounding motifs like NDSM (negatively charged amino acid-dependent SUMOylation motif), which is characterized by clusters of acidic residues in the downstream region (Yang et al., 2006), and PDSM (Phosphorylation- dependent SUMOylation motif) (Hietakangas et al., 2006) 
were identified to play a role in specificity and enhanced intrinsic propensities for SUMOylation.

\section{SUMO genes}

SUMO genes and other SUMOylation components are conserved among all eukaryotes. The budding yeast, Saccharomyces cerevisiae, contains a single SUMO (Smt3) protein which was shown to be essential for viability (Meluh and Koshland, 1995; Johnson and Blobel, 1997). The fission yeast, Schizosaccharomyces pombe, also contains a single SUMO gene (PMT3) whose deficiency results in defects in the genome maintenance but cells are viable (Tanaka et al., 1999). Similar to yeasts, invertebrates like C. elegans and Drosophila melanogastor, contain a single SUMO gene (Geiss-Friedlander and Melchior, 2007). Vertebrates contain four SUMO genes namely SUMO1, SUMO2, SUMO3 and the recently identified SUMO4 (Melchior, 2000; Geiss-Friedlander and Melchior, 2007). It was shown that SUMO2/3 as well as SUMO4 can form SUMO chains in vivo and in vitro (Tatham et al., 2001). SUMO 2/3 conjugation to substrate proteins is strongly induced in response to various stress conditions such as high temperature (Tempe et al., 2008). In plants, the monocot Oryza sativa contains three SUMO isoforms (Miura et al., 2007a), whereas the Arabidopsis genome contains nine SUMO genes named from AtSUMO1 to AtSUMO9 (Kurepa et al., 2003; Miura et al., 2007a). Among the nine SUMO genes in Arabidopsis, only four genes (AtSUMO1, 2, 3, and 5) are expressed (Kurepa et al., 2003; Saracco et al., 2007). AtSUMO4, AtSUMO6 and AtSUMO7 genes do not contain a Cterminal diglycine motif, while AtSUMO9 only contains a partial SUMO sequence, hence these genes were considered as pseudogenes (Kurepa et al., 2003; Saracco et al., 2007). In Arabidopsis, SUMO isoforms SUMO1/SUMO2 as well as SUMO3, were shown to form SUMO conjugates in vivo by using antibodies directed against SUMO1/SUMO2 (Kurepa et al., 2003; Lois et al., 2003) and SUMO3 (Kurepa et al. 2003). All of the SUMO isoforms are produced as precursor proteins, that must be processed by SUMO specific proteases to make the diglycine motif available for conjugation (Li and Hochstrasser, 1999). 


\section{SUMOylation pathway}

Mechanistically, the SUMO modification pathway like to the ubiquitination pathway involves a series of enzymes. Many of the SUMO pathway components show structural and functional homology to those of the ubiquitin pathway, but are very specific to SUMOylation (Melchior, 2000). The SUMO modification of target proteins is facilitated by three major enzymatic reactions mediated by E1 activating, the E2-conjugating and E3 ligase enzymes. SUMO detachment involves SUMO specific proteases belonging to the SENP [sentrin (also known as SUMO)-specific protease] family.

\section{SUMO E1 genes}

SUMO-activating E1 enzymes activate the mature SUMO proteins by acting at the Cterminal diglycine motif. Functional SUMO-activating enzyme (SAE) is a heterodimer formed from two proteins (SAE1 and SAE2) with molecular weights $\sim 40 \mathrm{kDa}$ and 70 kDa, respectively (Dohmen et al., 1995; Johnson et al., 1997; Desterro et al., 1999; Gong et al., 1999). SAE1 is similar to the amino-terminal half of ubiquitin activating enzyme, whereas SAE2 shows similarity to the carboxyl terminal half of the ubiquitin activating enzyme and contains the active-site cysteine (Melchior, 2000; Johnson, 2004). The Arabidopsis thaliana genome contains two genes for SAE1 protein namely, SAE1a (At4g24940) and SAE1b (At5g50580) (Colby et al., 2006; Miura et al., 2007a). The larger subunit of SAE, SAE2 is represented by a single copy gene (At2g21470) in the Arabidopsis genome (Colby et al., 2006; Miura et al., 2007a). The rice genome contains single genes for both SAE1 and SAE2 proteins (Miura et al., 2007a). The SUMOactivating enzyme (SAE) activates mature SUMO in a three step reaction. In the first step, SUMO C-terminal adenylate is formed and pyrophosphate is released by the reaction between the C-terminal carboxyl group of mature SUMO and ATP. Next, the thiol group of the active site in the SUMO activation enzyme attacks the SUMO adenylate, releasing AMP and forming a high energy thiolester bond between the E1 and the C terminus of SUMO. In the final reaction, the activated SUMO is transferred to a catalytic residue in the SUMO conjugation enzyme (SCE) (Johnson, 2004). Thus, at the end of activation reaction, SUMO is transferred to the SUMO E2 enzymes. 


\section{SUMO E2 genes}

The SUMO E2 enzymes are known as SUMO Conjugation Enzymes (SCE), which serve as the final donors of SUMO group to the substrate. Upon transfer from SAE, a SUMOE2 thiolester intermediate is formed between the active site of SUMO-conjugating enzyme (SCE) and C-terminal carboxyl group of activated SUMO (Johnson and Blobel, 1997; Desterro et al., 1999). Next, this intermediate attacks the target protein to form an isopeptide bond between the $\varepsilon$-amino group of an internal lysine residue in the SUMO consensus sequence and the activated SUMO carboxyl terminus (Johnson, 2004). This reaction is facilitated by the binding of SCE to the target protein. SCE proteins have a strong overall positive charge and a patch of protein surrounding the active site binds directly to the $\psi \mathrm{KXE} \backslash \mathrm{D}$ consensus sequence in the substrate (Bernier-Villamor et al., 2002; Tatham et al., 2003). Only one SCE is present in yeast (called UBC9), invertebrates, and most likely in vertebrates as well (Johnson, 2004). Unlike other model systems, plants contain multiple E2 enzymes. Arabidopsis and rice contains two and three E2 genes, respectively (Miura et al., 2007a).

\section{SUMO E3 genes}

SUMO ligases (E3 enzymes) facilitate the transfer of SUMO from the E2 enzyme to the target proteins. SUMO ligases can directly or indirectly bind to specific substrates to mediate SUMO transfer (Melchior, 2000; Johnson, 2004). Both in vitro and in vivo studies confirmed that E1 and E2 enzymes are sufficient for SUMOylation of some substrates (Desterro et al., 1999; Okuma et al., 1999). However, it is well recognized that E3 enzymes are necessary for most in vivo SUMO conjugating reactions (Johnson and Gupta, 2001; Takahashi et al., 2001; Pichler et al., 2002).

So far, four types of SUMO ligases have been identified in animals and fungi, namely SIZ/PIAS, RanBP2, Pc2, and NSE2/MMS21 (Johnson, 2004; Miura et al., 2007a). The SIZ-type of E3 ligases, which were the first to be identified and characterized, are similar to the major class of ubiquitin ligases in that they use a RINGlike domain for binding to SUMO protein (Hochstrasser, 2001; Jackson, 2001). These proteins contain SAP, PINIT, SP-RING, SUMO binding, and NLS domains (Sharrocks, 2006; Palvimo, 2007). SP-RING is a C2HC3- type of Zinc finger and aids in the binding 
of SIZ/PIAS proteins to SCE1. Both PINIT and SP-RING domains are necessary for SUMO E3 ligase activity of SIZ/PIAS proteins. The SAP domain binds to DNA and is important for trans-repression activity of PIASy protein, which attenuates transcription factor activity of STAT1 and the androgen receptor (Sharrocks, 2006; Palvimo, 2007). Plant SIZ/PIAS homologs contain another plant specific C4HC3 Zn-finger domain called the plant homeo domain (PHD), which also seems to function in DNA binding (Bienz, 2006; Miura et al., 2007a). Arabidopsis and rice contain two and three SIZ/PIAS homologues, respectively (Miura et al., 2007a).

RanBP2 (Ran-binding protein 2) and Pc2 do not show any sequence similarity to SIZ/PIAS type SUMO ligases or ubiquitin ligases. RanBP2 was first identified to interact with SUMOylated RanGAP at cytoplasmic filaments of the nuclear pore complex (Matunis et al., 1996; Mahajan et al., 1997). This type of SUMO ligase is probably restricted to animals, because its prominent substrate RanGAP is apparently not SUMOylated in fungi and a similar situation may occur in plants. In particular, the SUMO acceptor domain is lacking in plant RanGAP (Rose and Meier, 2001). So far, plant homologues of RANBP2 have not been identified. In contrast to RanBP, PcG proteins form large multimeric complexes, which are detectable microscopically as discrete foci, called PcG bodies within the cell nucleus (Kagey et al., 2003; Wotton and Merrill, 2007). Plant homologues of PcG proteins with SUMO ligase activity have not yet been identified.

NSE2/MMS21 (non-SMC element/methyl methanesulfonate sensitive) orthologs are characterized by SP-RING domains of the C2HC2-type that are necessary for SUMO E3 ligase activity. Single putative orthologs of NSE2/ MMS21 were identified in Arabidopsis and rice based on sequence information (Miura et al., 2007a).

\section{SUMO proteases}

The desumoylation process involves cleavage of SUMO from its target proteins, which is catalyzed by SUMO proteases via their SUMO isopeptidase activity. SUMO proteases maintain SUMO equilibrium by providing a source of free SUMO for conjugation of other proteins (Melchior, 2000; Geiss-Friedlander and Melchior, 2007). SUMO proteases contain a $~ 200$ amino acid domain (Ulp domain), which has the SUMO cleaving activity 
(Mossessova and Lima, 2000). The Ulp domain is distantly related to a number of viral proteases, but is not related to ubiquitin proteases (Li and Hochstrasser, 1999). Yeast SUMO proteases, Ulp1 and Ulp2 (ubiquitin-like modifier proteases) can catalyze the carboxyl terminus processing of SUMO and also remove SUMO from isopeptide linked conjugates ( $\mathrm{Li}$ and Hochstrasser, 2000). In mammals, seven proteins were identified with Ulp domains and are called SENPs (sentrin proteases). The homologs of SUMO proteases were identified in Arabidopsis and rice (Kurepa et al., 2003; Miura et al., 2007a) In Arabidopsis, 67 genes encode a domain similar to the SUMO protease domain have been identified. Thus, there has been a huge expansion in this class of proteases, and it is unlikely that all of them are specific for SUMO (Novatchkova et al., 2004). Arabidopsis AtULP1a, AtULP1c, AtULP1d, and AtESD4 have been functionally characterized as SUMO proteases, because they have SUMO peptidase and isopeptidase activities that are required for SUMO precursor protein processing and substrate deconjugation, respectively (Miura et al., 2007a).

\section{Regulation of SUMOylation system}

SUMOylation is a very dynamic process with only a small proportion of any protein being modified (Guo et al., 2007). It has been observed that some SUMO targets are modified constitutively, whereas many proteins are SUMOylated in a temporally or spatially regulated fashion (Bossis and Melchior, 2006b; Guo et al., 2007). It was proposed that SUMOylation can be regulated in four different ways as described below (Guo et al., 2007; Liu and Shuai, 2008).

\section{1) By altering the gene expression of SUMO cascade components}

The expression of various SUMO cascade components was shown to be regulated in response to different physiological and pathogenic conditions (Bossis and Melchior, 2006b; Liu and Shuai, 2008). It was also observed that during development, SUMO components are differentially expressed. This regulation of expression was shown to be affected by signaling mechanisms like $\mathrm{Ca} 2+$ signaling, cAMP and progesterone signaling (Jones et al., 2006; Deyrieux et al., 2007). During stress conditions like hypoxia, the expression of SUMO1 and RESUME (a SUMOylation enhancer protein) were promoted 
(Comerford et al., 2003; Carbia-Nagashima and Arzt, 2004). Elevated expression of human E2 enzyme UBC9 was observed under a number of human malignancies (Baek, 2006). However, the transcription factors involved in the regulation of SUMOylation components during stress or development have not been identified (Liu and Shuai, 2008).

\section{(2) By employing cross-talk with other post-translational modifications}

The lysine residue to which the SUMO protein is added, can also be modified by other post-translational modifications, including acetylation, methylation and ubiquitination, which provide opportunity for regulatory cross-talk between different pathways (Bossis and Melchior, 2006b). SUMOylation and acetylation were recognized as competing mechanisms in some proteins such as Sp3, p300, (Sapetschnig et al., 2002; Bouras et al., 2005). Antagonism was observed between SUMOylation and ubiquitination in proteins like IkBa, PCNA, where SUMOylation protects the proteins from ubiquitin mediated protein degradation (Desterro et al., 1998; Watts, 2006). Phosphorylation may serve both as a positive and a negative signal for SUMOylation. In some proteins, a phosphorylation dependent SUMOylation motif, called PDSM (YKXEXXSP), was identified downstream from the core SUMOylation motif which promotes SUMOylation by imparting a negative charge (Hietakangas et al., 2006). In some proteins, phosphorylation promotes SUMOylation independent of PDSM. Phosphorylation of proteins like p53, ELK-1, c-Jun and c-Fos reduces their capability of being SUMOylated (Muller et al., 2000; Yang et al., 2003; Lin et al., 2004; Bossis et al., 2005).

\section{(3) By regulating the enzyme activity of proteins involved in SUMOylation}

SUMOylation can be regulated in a more global way by directly targeting the basic conjugation or deconjugation machineries (Bossis and Melchior, 2006b). In contrast to the ubiquitin pathway, there is only a single E2 protein in the SUMOylation pathway, whose regulation can lead to a global response, as illustrated for Ubc9 activity by $\mathrm{H}_{2} \mathrm{O}_{2}$ (Bossis and Melchior, 2006b; Guo et al., 2007). During viral infection, expression of viral proteins like Gam1 in cells leads to a drastic decrease in cellular SUMO conjugates as a result of the disappearance of SUMO E1 and E2 enzymes (Boggio et al., 2004; Boggio et al., 2007). As SUMOylation globally represses signal transduction and gene expression, 
the virus promotes the transcription capacity of the cell, facilitating its own propagation (Boggio and Chiocca, 2005). Similarly, stress conditions like heat shock, osmotic and high oxidative stress $\left(\mathrm{H}_{2} \mathrm{O}_{2}\right)$, enhance global SUMOylation in mammals, plants and yeast (Saitoh and Hinchey, 2000). Conversely, reductions in global SUMOylation levels can be seen when low doses of $\mathrm{H}_{2} \mathrm{O}_{2}$ cause the formation of reversible disulfide bond formation between the catalytic cystine of the E1 subunit Uba2 and the E2 Ubc9 (Bossis and Melchior, 2006a).

\section{(4) By regulating the recruitment of E3 ligases}

Since regulation of the E2 conjugating enzyme results in global changes in SUMOylation, E3 ligases were proposed to be the focal points for regulation that leads to control of SUMOylation of individual substrates (Muller et al., 2004; Guo et al., 2007). E3 ligase recruitment to individual target proteins can be affected by post-translational modifications. DNA-damaging agents cause phosphorylation of PC2- type E3 ligases, which enhance its ligase activity towards its substrate (Roscic et al., 2006). Genotoxic stress induces the recruitment of PIASy E3 ligase to its target protein NEMO (Mabb et al., 2006). SUMO conjugation can be fine-tuned by regulating the sub-cellular localization of SUMO E3 ligases (Bossis and Melchior, 2006b).

\section{Effects of SUMOylation at the molecular level}

At a molecular level, SUMOylation alters protein functions by masking and/or adding interaction surfaces, or by inducing conformational changes that result in altered interactions. As a consequence, a wide variety of downstream affects have been observed, including changes in localization, enzymatic activity, or stability (GeissFriedlander and Melchior, 2007).

\section{Effects on transcription factor activity}

SUMOylation was mainly implicated in transcriptional repression, however recent studies indicate that it also plays a role in the activation of transcription (Muller et al., 2004; Geiss-Friedlander and Melchior, 2007; Zhao, 2007a). SUMOylation mediates repression by enhancing the repression activity of repressors or co-repressors. Sometimes 
SUMOylation associates with the suppression of the function of the transcription activators or co-activators (Verger et al., 2003; Zhao, 2007a). SUMOylation can also affect the DNA binding capability of transcription factors, thereby affecting transcription as observed for the heat-shock transcription factor, HSF1 (Goodson et al., 2001). The effect of SUMOylation on protein-protein interactions of transcription factors with other activators or repressors could also lead to the activation or repression of transcription (Gill, 2003; Geiss-Friedlander and Melchior, 2007; Zhao, 2007a). SUMOylation also affects the enzymatic activities of proteins regulating gene expression such as DNA methyl transferases, histone deacetylases, histone acetyl transferases (Zhao, 2007a). In addition, histones, the core components of chromatin, also undergo SUMOylation, which resulting in the general repression of transcription (Iniguez-Lluhi, 2006; Nathan et al., 2006).

\section{Effect on protein localization}

The SUMOylation system was implicated in nucleo-cytoplasmic protein transport because RanGAP1, a GTPase activating protein for the nucleocytoplasmic transport protein Ran, was the first substrate for SUMOylation to be discovered. Unmodified RanGAP is cytoplasmic, whereas SUMO modified RanGAP is associated with the nuclear pore complex and strongly interacts with RanBP2, a SUMO E3 ligase (Matunis et al., 1996; Mahajan et al., 1997; Matunis et al., 1998). It was later observed that several substrates like FAK, Caspase-7 and caspase-8 localized to the nucleus upon their modification by SUMOylation (Zhao, 2007a)). The observation that fusing SUMO to a target protein resulted in its localization in the nucleus further strengthening the idea that SUMO serves as a nuclear signal (Huang et al., 2003). SUMOylation was also implicated in sub-nuclear localization of proteins to nuclear foci like PML bodies. SUMOylation of PML protein is essential for its localization to PML bodies and the integrity of PML bodies. Many other SUMO-modified proteins have also been found in PML nuclear bodies including transcription factors, chromatin modifiers, proteins involved in genomic maintenance, SUMO E3 ligases and SUMO-specific proteases have also been found in PML nuclear bodies (Zhong et al., 2000; Sachdev et al., 2001; Best et al., 2002; Gill, 2004; Zhao, 2007a). These observations indicate that SUMO modification mediates 
protein-protein interactions, which are important for the integrity of PML bodies. In fact, in some of the PML body associated proteins, a SUMO Binding Motif/SUMO Interaction Motif (SBM/SIM) was identified and further supports the role of SUMO in mediating protein-protein interactions (Minty et al., 2000; Hardeland et al., 2002; Lin et al., 2002). In addition, several proteins involved in SUMOylation such as E3 ligases (Nup358, RanBP2), E2 conjugation enzymes (Ubc9) and SUMO proteases (SENP2) were shown to be associated with the nuclear pore complex. This implies that proteins may be SUMOylated as they enter the nucleus, which may play a role in retention of these proteins in the nucleus (Matunis et al., 1998; Hang and Dasso, 2002; Zhang et al., 2002; Melchior et al., 2003). However, for some proteins like LEF1 and sp100, SUMO modification does not alter their localization (Sternsdorf et al., 1999; Sachdev et al., 2001). SUMOylation has also been implicated in promoting the nuclear export of proteins like Dictyostelium MEK1 (dMEK1) (Sobko et al., 2002). SUMOylation of DRP1 protein leads to its recruitment from cytosol to the mitochondrial outer membrane (Harder et al., 2004).

\section{SUMO modification and the control of genome integrity}

SUMO-1 mutants of yeast exhibited defects in chromosomal segregation and aberrant mitosis (Meluh and Koshland, 1995). Other SUMOylation components like E2 conjugating enzyme, E3 ligating enzymes and SUMO isopeptidases were also implicated in chromosomal segregation (Zhao, 2007a). SUMOylation of substrates like topoisomerase II (Top2) and Psds5 is important for chromatid cohesion (Bachant et al., 2002; Stead et al., 2003). Cenp-C is a target of SUMO1 and that plays a key role for mitotic progression at centromeres (Chung et al., 2004). SUMOylation was also implicated in the maintenance and recruitment of proteins to the kinetochore. SUMOylated RanGAP1 is targeted to both the microtubule spindle and kinetochores to guide their attachment during mitosis in He-La cells (Joseph et al., 2002; Joseph et al., 2004). In addition the SUMOylation of DNA repair proteins like PCNA, thymidine DNA glycosylase (TDG), RAD51/RAD52, and Smc5/6 was shown to affect their activity and therefore implicates a role in DNA repair (Andrews et al., 2005). 


\section{Effect on protein interactions}

SUMOylation can either promote or hinder protein-protein interactions. For example, SUMOylation of target proteins like RanGAP1, PCNA, p300 facilitates association with their interactors RanBP2, DNA helicase Srs2 and HDAC6, respectively (Mahajan et al., 1997; Matunis et al., 1998; Girdwood et al., 2003; Papouli et al., 2005). SUMOylation inhibits interaction of the proteins like ZNF6, E2-25k with their interactors (Zheng and Yang, 2004; Pichler et al., 2005). SUMOylation of proteins like RanGAP1, E2-25K and ETS1 do not influence the structure of either the SUMOylated target or of the SUMO modifier itself. Therefore, the loss or gain of interactions may result from simple masking or the addition of binding sites (Geiss-Friedlander and Melchior, 2007). SUMOylation of thymine DNA glycosylase (TDG) induces a conformational change in TDG, which ultimately results in a loss of DNA binding (Hardeland et al., 2002; Baba et al., 2005). Frequently, SUMO itself contributes to the association of target protein with a downstream effector. In proteins like PML, Daxx105, p73, a short motif was identified that interacts non-covalently with SUMO and is termed the SIM/SBM (SUMO interacting motif or SUMO binding motif) (Minty et al., 2000; Song et al., 2004; Hecker et al., 2006; Lin et al., 2006). The SIM/SBM motif plays an important role in the integrity of PML bodies (Shen et al., 2006) and contributes to the enzyme function for SUMO cascade components like UBA2, PIAS E3 ligases and RanBP2 (Song et al., 2004; Reverter and Lima, 2005). Recently, a family of ubiquitin ligases with SIM/SBM was identified to interact specifically with SUMO conjugated proteins and target them for proteasomal degradation. This indicated an indirect role for SUMoylation in protein degradation (Prudden et al., 2007; Sun et al., 2007; Uzunova et al., 2007).

\section{Effect on other post-translational modifications}

The SUMOylation of some proteins like $\mathrm{I} \kappa \mathrm{B} \alpha, \mathrm{Smad} 4$, Huntington, PCNA, and Rad52 prevents their degradation by ubiquitination (Desterro et al., 1998; Lin et al., 2003; Steffan et al., 2004; Papouli et al., 2005; Sacher et al., 2006). In some proteins, a single lysine residue can be either SUMOylated or ubiquitinated to prevent or promote proteasome-mediated protein degradation, respectively. In other proteins, even though the SUMOylation and ubiquitination sites may differ, these modifications still antagonize 
each other (Gill, 2004; Zhao, 2007a). Acetylation and SUMOylation play opposite roles on some proteins. SUMOylation was observed to prevent acetylation and promote deacetylation (Muller et al., 2001; Zhao, 2007a). For example, it was observed that acetylation or SUMOylation compete same lysine residues on histone proteins. Acetylation of histones promotes gene transcription, whereas SUMOylation represses gene expression (Shiio and Eisenman, 2003; Nathan et al., 2006). Several transcriptional factors like MEF2 PLAG1/PLAGL2, NF-IL6b and ELK-1 are cross-regulated by SUMOylation and acetylation/deacetylation (Zhao, 2007a). Unlike ubiquitination and acetylation, SUMOylation and phosphorylation can have a positive or negative influence on each other. Phosphorylation inhibits the SUMOylation of proteins like IкB $\alpha$ and AIB1 (Desterro et al., 1998; Wu et al., 2006). However, phosphorylation of many substrates like MEF2C, MEF2D and HSF1 facilitates their SUMOylation (Hietakangas et al., 2003; Gregoire et al., 2006; Kang et al., 2006). In some proteins, a phospho-sumoyl switch, or PDSM (phosphorylation dependent sumoylation motif, yKxExxS/T) is observed, which facilitates SUMOylation (Hietakangas et al., 2006; Yang et al., 2006).

Extensive studies on the SUMOylation system in different model organisms have indicated a critical role in development. SUMOylation has also been implicated in various stress responses including in plants. However, compared to yeasts and animals, very few SUMOylation target proteins have been identified in plants limiting our knowledge regarding the molecular effects of SUMOylation. Even though SUMOylation has been implicated in plant development and stress responses, it is not known how the SUMOylation system is regulated. In this study, we identified plant CSPs as targets for SUMO modification and monitored the SUMO pathway transcript levels in rice during development and after exposure to different stress conditions. 


\title{
Chapter 1: Functional characterization of two cold shock domain proteins from Oryza sativa
}

\author{
Published as
}

Vijay Chaikam and Dale Karlson*

Plant Cell Env (2008), 31, 995-1006

West Virginia University, Division of Plant \& Soil Sciences, 1090 Agricultural Sciences, Morgantown, WV 26506-6108, USA

*Present address: Monsanto Company, RTP, NC 27709, USA. 


\begin{abstract}
Two novel rice cold shock domain (CSD) proteins were cloned and characterized under different stress treatments and during various stages of development. OsCSP1 and OsCSP2 (Oryza sativa CSD protein) encode putative proteins consisting of an N-terminal $\mathrm{CSD}$ and glycine-rich regions that are interspersed by 4 and $2 \mathrm{CX} 2 \mathrm{CX} 4 \mathrm{HX} 4 \mathrm{C}$ (CCHC) retroviral-like zinc fingers, respectively. In vivo functional analysis confirmed that OsCSPs can complement a cold-sensitive bacterial strain which lacks four endogenous cold shock proteins. In vitro ssDNA binding assays determined that recombinant OsCSPs are capable of functioning as nucleic acid-binding proteins. Both $O s C S P$ transcripts are transiently up-regulated in response to low-temperature stress and rapidly return to a basal level of gene expression. Protein blot analysis determined that OsCSPs are maintained at a constant level subsequent to a cold treatment lasting over a period of several days. Both the transcript and protein data are in sharp contrast to those previously obtained for winter wheat WCSP1. A time-course study through various stages of rice development confirmed that both OsCSP proteins and transcripts are highly accumulated in reproductive tissues and tissues which exhibit meristematic activity.
\end{abstract}




\section{INTRODUCTION}

The adaptation of plants to low-temperature stress is a very important physiological factor affecting their geographical distribution and overall productivity in various climates. Within the plant kingdom, plants' ability to tolerate low-temperature stress is highly variable. Some plant species can acquire tolerance to low temperature subsequent to a period of exposure to low but non-freezing temperatures, a process which is termed cold acclimation. Within monocots, rice and wheat are two representative genera exhibiting clear differences in their tolerance to low-temperature stress. Winter wheat acclimates to low temperatures and can survive exposure to freezing temperatures, whereas rice is unable to acclimate. Although substantial progress has been made towards understanding the molecular mechanisms which govern cold acclimation in plants (Thomashow, 1999; Viswanathan and Zhu, 2002; Xiong et al., 2002; Chinnusamy et al., 2006; Zhu et al., 2007), the entire mechanism is not completely understood. Therefore, it is of great interest to resolve the disparity between tolerant and sensitive species in an effort to decipher critical components of cold acclimation that have not yet been identified.

CSPs are well-conserved nucleic acid-binding proteins that play a critical role for the adaptation of bacteria to low-temperature stress. The function of CSPs during lowtemperature stress has been extensively studied in prokaryotes; however, this relationship is poorly understood in eukaryotes and plants. Bacterial CSPs are small (67-73 amino acids) and consist of a single nucleic acid-binding CSD, which contains two consensus RNA-binding motifs (RNP1 and RNP2) (Landsman, 1992; Schindelin et al., 1993; Manival et al., 2001). Within the nine-member Escherichia coli Csp protein family, four members are induced upon low-temperature stress with the predominant CspA accumulating up to $10 \%$ of the total soluble protein fraction (Goldstein et al., 1990; Lee et al., 1994; Nakashima et al., 1996; Wang et al., 1999). When a quadruple deletion of CSPs was created in an E. coli strain (BX04), these cells acquired sensitivity to lowtemperature downshifts (Xia et al., 2001). Bacterial CSPs function as transcription antiterminators and RNA chaperones under low-temperature stress (Jiang et al., 1997; Hanna and Liu, 1998; Bae et al., 2000; Phadtare et al., 2002). 
In higher eukaryotes, such as vertebrates, CSD proteins have been commonly referred to as 'Y-box' proteins (Didier et al., 1988). Unlike bacterial CSPs, Y-box proteins contain various auxiliary C-terminal domains in addition to an N-terminal CSD. Y-box proteins play various roles in DNA metabolism, transcription and translation, and may promote DNA recombination and repair (Ise et al., 1999; Skabkin et al., 2001; Gaudreault et al., 2004). By acting as a transcription factor, YB-1 affects gene expression either positively or negatively of many important cellular genes. Concomitant with transcription, YB-1 associates with mRNA and affects alternative splicing in the nucleus (Chansky et al., 2001; Stickeler et al., 2001) and translation in the cytoplasm (Minich and Ovchinnikov, 1992). Collectively, these extensive studies have demonstrated that CSD proteins from higher eukaryotes perform diverse functions. However, the functional relationship of CSD proteins to low-temperature stress in eukaryotes is not clear. To our knowledge, only one report has demonstrated that chicken YB-1 is important for cellular growth under reduced temperatures (Matsumoto et al., 2005).

A novel form of eukaryotic CSD proteins was identified in plants and was described to contain two separate nucleic acid-binding modules: a single N-terminal CSD and a variable number of C-terminal retroviral-like CCHC type zinc fingers (Kingsley and Palis, 1994). In a subsequent study, plant CSD proteins were shown to be highly conserved among various plant genera (Karlson et al., 2003). The first functional analyses for a plant CSD protein were performed with WCSP1 from winter wheat. WCSP1 accumulates in response to low-temperature stress and exhibits nucleic acidbinding activity (Karlson et al., 2002). Subsequent experiments in Arabidopsis, which is also capable of cold acclimation, confirmed that its CSD proteins differentially respond to low-temperature stress on a transcript level (Karlson and Imai, 2003; Fusaro et al., 2007; Kim et al., 2007). It was recently shown that plant CSD proteins possess similar in vitro and in vivo functions to those of bacterial CSD proteins (Nakaminami et al., 2006; Kim et al., 2007). However, the precise function of plant CSD proteins has not yet been identified in relation to low-temperature stress in planta.

Apart from their role in cold adaptation, CSD proteins are also implicated in various aspects of development in different model systems. In the quadruple deletion mutant strain (BX04) of E. coli, cell division defects were observed and cells became 
elongated under cold stress (Xia et al., 2001; Kim et al., 2007). In mice, a knock-out for YB-1 resulted in embryo lethality (Lu et al., 2005; Uchiumi et al., 2006). A reduction of MSY2 levels in mouse oocytes results in reduced fertility (Yu et al., 2004). Another CSD protein (Lin28) from the Caenorhabditis elegans controls developmental transitions during early stages (Moss et al., 1997).

In plants, an Arabidopsis CSD protein (AtGRP2) was recently proposed to play an important role in plant development. Suppression of the AtGRP2 gene resulted in various developmental abnormalities with respect to flowering time, apical dominance and seed development. The transcripts of this gene were highly expressed in meristematic areas where rapid cell divisions occur (Fusaro et al., 2007). It was suggested that this protein might function in the regulation of specific mRNAs during development. Despite this advance, the functional role of CSD proteins in plant development is poorly understood. To understand the collective function of plant CSD proteins in the adaptation to low-temperature stress and during development in a crop plant, we initiated the first functional analysis of CSD proteins from rice. In the present study, I describe the in vitro and in vivo functions of OsCSPs. To further understand the putative role of these proteins in the functional context of plants, expression studies were conducted under various stress treatments and during different stages of development on both the gene and protein level.

\section{MATERIALS AND METHODS}

\section{Plant material and stress treatments}

Rice seeds of the Japonica cultivar Yukihikari were initially surface sterilized with three separate 5 min washes with $96 \%$ ethyl alcohol. The seeds were subsequently treated with $1.5 \%$ sodium hypochlorite for $30 \mathrm{~min}$. Following the sodium hypochlorite treatment, the seeds were washed thoroughly with water to remove excess sodium hypochlorite. The seeds were then imbibed with water for $3 \mathrm{~d}$ at $25^{\circ} \mathrm{C}$ in a growth chamber under continuous light conditions. Germinated seeds were spread evenly atop a mesh grid and placed above a plastic container filled with tap water. The seedlings were maintained in a growth chamber for one week at $25^{\circ} \mathrm{C}$. The seedlings were subsequently transferred to a $4^{\circ} \mathrm{C}$ chamber containing pre-cooled water. For gene expression studies, tissues were 
collected over a $24 \mathrm{~h}$ time period. For protein expression studies, tissues were collected over a $10 \mathrm{~d}$ period of cold treatment. A recovery sample was also collected $2 \mathrm{~d}$ after the samples were returned to a normal growth temperature $\left(25^{\circ} \mathrm{C}\right)$. For studying the effects of salt and abscisic acid (ABA) on transcript levels, 1 week seedlings were transferred to $250 \mathrm{mM} \mathrm{NaCl}$ solution and $50 \mu \mathrm{M}$ ABA solution, respectively. Tissue was collected over $24 \mathrm{~h}$ time period subsequent to the transfer. For imposing a drought stress, water was removed from the container. Excess water was wicked away from seedling roots with a paper towel. Tissue samples were taken over a time period of $24 \mathrm{~h}$ subsequent to the removal of water.

\section{Plant material and developmental series}

For developmental expression studies, seeds were surface sterilized as described earlier and germinated for $3 \mathrm{~d}$ in a $25^{\circ} \mathrm{C}$ growth chamber under continuous light conditions. At this stage, radicles, hypocotyls and seeds were separated from germinating seeds and used for the extraction of proteins. The remaining seeds were sown in pots and grown in a glasshouse at West Virginia University (April-July 2006). Growing media and fertilizer compositions were prepared as previously described (http://ricelab.plbr.cornell.edu/docs/CONDITIONS FOR GROWING RICE.doc).

Briefly, soil medium was prepared from peat, vermiculite, lime and Peters Unimix PlusIII (Scotts, Marysville, OH, USA). The plants were irrigated daily from the top of the soil surface, and pots were fertilized with Peters water-soluble fertilizer (N.P.K-15.16.17) once a week. Iron supplementation was provided every other week. After booting, the plants were only watered with fertilizer which lacked iron. For the collection of tissue, seedlings were removed from soil at the first-week stage. Roots were thoroughly washed with tap water and aerial tissues were collected separately. At this time point, all aerial tissues were considered as shoots and were collectively harvested. After 1 month of growth during the tillering stage, roots, crowns and leaves were collected separately. At this stage, stem tissue refers to the $0.5-1.0 \mathrm{~cm}$ region just above the crown tissue. Similar tissue profiling was performed during the panicle development stage where the majority of tillers contained panicles of different sizes. Panicles were grouped into four categories based upon length as follows: $<2,2-5,5-10$ and $>10 \mathrm{~cm}$. Flowers were collected from 
mature panicles before and after the opening of flowers. Seeds were collected at the milk and dough stages. All of the tissues were frozen in liquid nitrogen and stored at $-80^{\circ} \mathrm{C}$ until further use for RNA and protein extractions.

\section{Recombinant protein production/purification and ssDNA binding assay}

The complete open reading frames of $O s C S P 1$ and $O s C S P 2$ were cloned in-frame into the pET43.1a vector for an N-terminal fusion to NusA protein and a C-terminal 6X Histag. In-frame 5'EcoRI sites were added with the following primers for OsCSPI and OsCSP2, respectively: 5'-TCTGAATTCATGGCGTCGGAGAGGGTG-3', 5'TCTGAATTCATGGCGGCGGCGGCGAGG-3'. XhoI sites were incorporated immediately after the last codon and facilitated an in-frame C-terminal fusion to a $6 \mathrm{X}$ His-tag. The respective primers for adding XhoI to OsCSP1 and OsCSP2 were as follows: 5'-TCTCTCGAGGTAGGTCT TGCTGGGGCAC-3', 5'-TCTCTCGAGCTTGTGGCAG TCGCGGGC-3'. WCSPl was similarly cloned by adding 5'BamHI and 3'XhoI sites. PCR products were amplified with Takara La-Taq with GC-buffer II and subsequently purified with a Qiagen PCR clean-up kit (Qiagen, Valencia, CA, USA). Purified PCR products were digested overnight with EcoRI and XhoI, and ligated into a pre-digested pET-43.1a vector. Constructs were confirmed for sequence integrity and transformed into BL21DE3 cells (Novagen, Madison, WI, USA) for the production of recombinant proteins. The transformed colonies were grown in Luria-Bertani (LB)-carbenicillin liquid medium until the OD600 reached 0.6, and recombinant proteins were induced by adding isopropyl $\beta$-D-1-thiogalactopyranoside (IPTG) to a final concentration of $1 \mathrm{mM}$. Cultures were subsequently grown for an additional $3 \mathrm{~h}$ at $37^{\circ} \mathrm{C}$. Cells were pelleted and resuspended in BugBuster Protein Extraction Reagent (Novagen) with the addition of benzonase nuclease (Novagen) according to the supplier's instructions. Soluble proteins were collected from the supernatant fraction after spinning at $20,000 \mathrm{~g}$ for $20 \mathrm{~min}$ in a refrigerated centrifuge. Soluble fractions were applied to Ni-NTA matrix beads and purified by a batch method. Recombinant fusion proteins were eluted from the columns according to the supplier's instructions (Qiagen). Eluates were applied to Centricon YM-3 (Millipore, Billerica, MA, USA) spin columns for buffer exchange with 3 volumes of icecold $50 \mathrm{mM} \mathrm{NaPO} 4$ buffer. Samples were subsequently quantified by using Bradford 
assay reagent (Bio-Rad, Hercules, CA, USA). The purity of the recovered proteins was assessed with sodium dodecyl sulphate-polyacrylamide gel electrophoresis (SDS-PAGE) according to standard procedures. Nucleic acid-binding analyses to ssDNA were performed as previously described (Karlson et al., 2002), with the exception that proteins were applied at a lower concentration range of 10-100 pM. NusA protein, which was also tagged with a $6 \mathrm{X}$ His-tag, was purified and applied at the highest concentration (100 pM) to serve as a negative control for the ssDNA binding assay.

\section{Complementation assay}

The coding regions for OSCSP1 and OsCSP2 were cloned in-frame into an inducible pINIII vector by adding N-terminal NdeI sites with the following primers, respectively, 5'-TCTCATATGGCGGCGGCGGCGAGGCATCGGGGG-3' 5'TCTCATATGGCGTCGGAGAGGGTGAAGGGGACG-3'. C-terminal EcoRI sites were created by PCR amplification from primers 5'-TCTGAATTCTCACTT GTGGCAGTCGCGGGCGAT-3' and 5'-TCTGAATTCC TAGTAGGTCTTGCTGGGGCACTC-3' for OsCSP1 and OsCSP2, respectively. WCSP1 was similarly cloned by adding 5' $\mathrm{NdeI}$ and 3'EcoRI sites. Constructs were confirmed for the correct translational frame and for sequence integrity. Plasmids were subsequently transformed into BX04 cells for the in vitro complementation assay as previously described (Nakaminami et al., 2006). In brief, two colonies for each construct were grown in LB-carbenicillin liquid medium until an OD600 of 1.4 was reached. The samples were then diluted to an OD of 0.1 and were further diluted 100 times for spotting on two replica LB-carbenicillin plates that were supplemented with IPTG. A control plate was grown overnight at $37^{\circ} \mathrm{C}$, and the additional plate was incubated at $15^{\circ} \mathrm{C}$ for observation of bacterial growth over a period of $7 \mathrm{~d}$.

\section{Gene expression analyses}

Low-temperature stress treatments were performed in a hydroponic setting as previously described (Karlson et al., 2002). Briefly, seedlings were harvested prior to and 1, 2, 6, 12 and $24 \mathrm{~h}$ subsequent to the initiation of stress treatments. For cold treatment, additional time points were included within the first $2 \mathrm{~h}$. Total RNA was isolated from rice root and 
shoot tissues using TRIzol reagent (Invitrogen, Carlsbad, CA, USA) according to the supplier's instructions. One microgram of isolated total RNA was converted into cDNA by application of the QuantiTect reverse transcription kit (Qiagen). The prepared cDNA was diluted 100 times and used as a template for qRT-PCR or semiquantitative reverse transcription PCR (RT-PCR). Triplicate qRT-PCR reactions were performed on all diluted cDNA samples with a Bio-Rad iCycler with the iQ SYBR Green Supermix (BioRad) by applying standard cycle temperatures. Primers were designed to amplify a 150bp region from the 3'UTR regions of $O s C S P 1$ and $O s C S P 2$. The primer pairs were 5'CTGCTCGTGTGATGATGCTT-3' and 5'-CTTTTTCATCTCG GGGAAAC-3' for OsCSP1 and 5'-GCGGGTGATCAGTTCTATCTT-3' and 5'GTCTCTCAAACCGACCCAAC-3' for OsCSP2,. The 18SrRNA gene was used as a housekeeping gene for the normalization of qRT-PCR reactions. The following primer pair selectively amplified $18 S r R N A$ as a $150 \mathrm{bp}$ product: 5'-AAGACGAA CAACTGCGAAAG-3' and 5'-GGCGGAGTCCTATAA GCAAC-3'. qRT-PCR results were analyzed using the relative standard curve method.

For semiquantitative RT-PCR, a different set of primers was used to amplify a larger 300 bp product. The primer sequences were as follows: 5'-ACCATCTCCCCCCCC CCTCC-3' and 5'-CTTTTTCATCTCGGGGAAAC-3' for OsCSP1 and 5'TGCTACAACTGCGGCGAGACCGGCC-3'

and 5'ATTTTCTTCTCGGTTCATAATAGAT-3' for OsCSP2, respectively. The identical primer set for $18 S r R N A$ was used for semiquantitative RT-PCR.

\section{Plant protein extraction and Western blotting}

Rice tissues that had been harvested during different developmental stages were ground with liquid nitrogen and subsequently boiled for 5 min with SDS-PAGE sample buffer. The extracted total proteins were centrifuged at $14000 \mathrm{rpm}$ for $5 \mathrm{~min}$ to remove plant debris. The isolated proteins were quantified by applying a modified Bradford protein assay as previously described (Karlson and Imai, 2003). Fifteen micrograms of each sample was separated by SDS-PAGE and subsequently transferred to nitrocellulose membranes for western blot analysis. Anti-WCSP polyclonal antibodies (1:1000) and alkaline phosphatase conjugated anti-rabbit secondary antibodies (1:3000) were used for 
the detection of rice CSD proteins with the NBT-BCIP reagent (Pierce, Rockford, IL, USA)

\section{RESULTS}

\section{DNA sequence analysis}

Full-length clones were PCR amplified from isolated cDNA template and found to contain sequences identical to those described from the rice genomic database. In this report, I have subsequently named the two rice CSD protein homologs OsCSP1 (Os02g0121100) and OsCSP2 (Os08g0129200). Complete bidirectional sequence analysis determined that the open reading frames for OsCSP1 and OsCSP2 contain 726 bp and $594 \mathrm{bp}$, and encode putative 22.7 and $18.7 \mathrm{kDa}$ glycine-rich proteins, respectively. Both rice homologs contain N-terminal CSDs and glycine-rich regions that are interspersed with four and two carboxy-terminal CCHC-type zinc fingers for OsCSP1 and OsCSP2, respectively (Fig. 1-1). Putative translated products of OsCSP1 and OsCSP2 contain $37 \%$ glycine and $40 \%$ glycine residues, respectively. Hydropathy plot analyses revealed that both OsCSP1 and OsCSP2 are predominantly hydrophilic (not shown).

\section{OsCSPs exhibit nucleic acid-binding activity}

The nucleic acid-binding activity of CSD proteins is a critical component of their in vivo functional roles. Thus, I tested if rice CSD proteins are also capable of functioning as nucleic acid-binding proteins. For this purpose, recombinant OsCSP1 and OsCSP2 proteins were produced as fusions to an N-terminal NusA tag and a C-terminal 6X Histag. Because NusA is extremely soluble, this tag enhanced the capacity for our recombinant OsCSP proteins to become enriched in the soluble protein fraction. Highly purified recombinant proteins were visualized as single bands with SDS-PAGE analysis (Fig. 1-2a). Equally loaded recombinant proteins were added to $150 \mathrm{ng}$ of M13mp8 ssDNA at increasing concentrations. NusA protein was employed as a negative control and was added at the highest concentration (100 pM). The migration of DNA was clearly retarded when increasing concentrations of OsCSP1 and OsCSP2 were added to the reaction mixture. Conversely, when purified NusA protein was applied at the highest 
concentration, no evidence of DNA gel shifting was detected (Fig. 1-2b). These data confirmed that OsCSPs are capable of binding nucleic acids.

\section{OSCSPs complement a cold-sensitive E. coli mutant}

To determine if OsCSPs are capable of functioning in a similar fashion to their prokaryotic counterparts in vivo, I performed a functional complementation study using the BX04 mutant which contains a quadruple deletion of four endogenous CSPs. OsCSPs were cloned into the IPTG-inducible pINIII expression vector. Cloned constructs were verified for sequence integrity and were subsequently transformed into BX04 competent cells. All transformants, including negative (pINIII empty vector) and positive controls (CspA, WCSP1), clearly grew at $37^{\circ} \mathrm{C}$ (Fig. 1-3). However, when transformants were exposed to prolonged low temperature $\left(15^{\circ} \mathrm{C}\right)$, only cells transformed with OsCSPs and the positive controls of CspA and WCSP1 exhibited growth (Fig. 1-3).

\section{Response of OsCSPs to low-temperature stress}

To study the effect of low-temperature stress on OSCSP expression, rice seedlings were treated with $4{ }^{\circ} \mathrm{C}$, and the response of OsCSPs was characterized at the transcript and protein level by applying qRT-PCR and western blotting, respectively. qRT-PCR analyses revealed that $O S C S P S$ gene transcripts are present in both roots and shoots under normal growth conditions. Upon treatment with low temperature, the accumulation of mRNA of OsCSPs in root (Fig. 1-4a) and shoot tissues (Fig. 1-4c) is marginally and transiently increased. This transient increase peaks approximately $30 \mathrm{~min}$ and $1 \mathrm{~h}$ after

the transfer to low temperatures in root and shoot tissues, respectively. Usage of a positive control cold-responsive gene (OsLIP19) (Wen et al., 2002) confirmed that our experimental conditions were properly executed for both roots (Fig. 1-4b) and shoots (Fig. 1-4d). Relative comparisons to OsLIP19 revealed that both OsCSPs have very low mRNA levels. 


\section{Response of OsCSPs to various abiotic stresses}

In addition to low-temperature stress, I was interested to characterize the response of OsCSPs to a variety of abiotic stresses. OsCSP expression patterns were determined after rice plants were treated with drought, salt and ABA (Fig. 1-5). Similar to expression under cold stress, a transient and marginal increase of OsCSPs was also noted in the root tissue, when treated with drought and ABA. A very slight increase was noted for OsCSPI when treated with salt stress. No distinguishable response patterns were observed for OsCSPs in shoot tissue during drought and salt stress. Both OsCSPs appear to respond transiently in shoot tissue when exposed to exogenous ABA. Usage of a stress-responsive positive control gene Os01g0348900 (jacalin-related lectin domain containing protein) (Rabbani et al., 2003) confirmed that all of our treatments were properly executed (data not shown).

\section{OsCSPs are detected with WCSP1 polyclonal antibody}

A polyclonal antibody was generated against the CSD region of WCSP1 in the laboratory of Dr. Ryozo Imai. Prior to performing any western blot analyses with rice total protein extracts, I confirmed whether this antibody could effectively recognize both OsCSP1 and OsCSP2. Recombinant NusA::OsCSP fusion proteins were spotted onto a nitrocellulose membrane. In addition, recombinant NusA::WCSP1 and NusA proteins were spotted as positive and negative controls, respectively. The membrane was stained with Ponceau S to confirm equal concentration of spotted proteins (Fig. 1-6a). The dot blot assay revealed that both OsCSPs are recognizable by the anti-WCSP1 antibody (Fig. 1-6b). The detected signal was greatest from the spotted WCSP1 protein, and the level of detection for OsCSP1 was at relatively comparable level. However, the OsCSP2 protein was recognized at a comparatively lower level. The negative control NusA protein was not recognized by this antibody, indicating that the antibody was specific to CSD proteins (Fig. 1-6b). Similar results were obtained when the recombinant proteins were run on an SDS-PAGE gel and subjected to western blot analysis (Fig. 1-6c). After confirming that this antibody could detect both OsCSPs, I proceeded with western blot analyses of protein extracts isolated from a low-temperature stress treatment and from various developmental stages. 


\section{Response of OsCSP proteins to low-temperature stress}

Western blot analysis of total protein extracts harvested from rice crown tissue did not reveal any accumulation of OsCSPs over the $10 \mathrm{~d}$ period of the low-temperature treatment (Fig. 1-7). These data were in sharp contrast to those previously reported for winter wheat WCSP1. When exposed to low-temperature stress, WCSP1 protein exhibits a dramatic accumulation during a low-temperature treatment (Karlson et al., 2002).

\section{Developmental expression patterns of OsCSP proteins}

To determine if OsCSPs are developmentally regulated, total protein extracts were prepared from various tissues during different developmental stages and subjected to western blot analysis with the WCSP1 polyclonal antibody. OsCSPs exhibited a clear trend for accumulation in reproductive tissues such as panicles, flowers and seeds. OsCSPs accumulate during panicle development and appear to decrease once panicles are greater than $10 \mathrm{~cm}$ in length (Fig. 1-8). In flowers and seeds, OsCSP1 is highly expressed and appears to decrease with the progression of maturity. Although the relative level is less, OsCSP2 is also detected during the reproductive stage as well. During the early vegetative stages, OsCSPs accumulated in tissues which contain an abundance of actively dividing cells like radicles, hypocotyls and crown tissue (Fig. 1-8). To provide an additional level of correlation to the developmental pattern of OsCSP protein accumulation, I monitored the expression of $O s C S P s$ with semiquantitative RT-PCR. The expression data were in good accordance with the western blot data. In comparison to a vegetative flag leaf, OsCSP transcripts were enriched in the reproductive tissues like panicles, flowers and seeds (Fig. 1-9).

\section{DISCUSSION}

Bacterial CSPs are highly induced during cold shock response and are critical for the adaptation of bacterial cells to cold stress (Xia et al., 2001). The important function of CSPs is based on their RNA chaperone activity which destabilizes the secondary structures that form during cold exposure (Bae et al., 2000; Phadtare et al., 2002). CSD proteins are present in almost all living organisms from bacteria to higher vertebrates and 
plants. In plants, winter wheat and Arabidopsis CSD proteins were recently implicated to play a role in cold adaptation because they are capable of performing similar in vivo and in vitro functions as bacterial CSPs (Nakaminami et al., 2006). However, the function and their respective modes of action for these proteins have not been well studied in planta.

CSD proteins are induced under cold stress conditions in plants that are capable of cold acclimation, such as winter wheat and Arabidopsis (Karlson et al., 2002; Karlson and Imai, 2003). These correlative data provide evidence suggesting that plant CSD proteins may play an important role during cold acclimation. Many tropical and subtropical plants are highly susceptible to low-temperature stress and cannot undergo cold acclimation. To gain a better understanding of the potential role of plant CSD proteins during cold acclimation, I functionally characterized CSD proteins from rice, an agriculturally important crop, which cannot acclimate to low temperatures. This comparative analysis enabled us to begin to assess the potential significance of the lowtemperature regulation of CSD proteins during cold acclimation.

Rice contains two unique CSD genes located on chromosomes 2 (OSCSP1; Os02g0121100) and 8 (OsCSP2; Os08g0129200). Similar to other plant CSD proteins, OsCSPs contain three distinct domains: an N-terminal CSD and a glycine-rich domain that was interspersed with $\mathrm{CCHC}$ type retroviral-like zinc finger domains. Within the CSD region, OsCSP1 and OsCSP2 are 68\% identical to each other. Both are approximately 48\% identical to bacterial CspA. This similarity reflects the highly conserved nature of the CSD as previously reported (Karlson and Imai, 2003). The two rice CSPs differ in their C-terminal domains with OsCSP1 containing four zinc fingers and OsCSP2 containing two zinc fingers (Fig. 1-1). This combination of two different DNA binding modules, the CSD and zinc fingers, has been widely noted in plant CSD proteins (Kingsley and Palis, 1994; Karlson and Imai, 2003). A similar modular structure with an N-terminal CSD and C-terminal CCHC zinc fingers was identified in a CSD protein from C. elegans (LIN-28), which was found to be critical for developmental timing (Moss et al., 1997).

The CSD, which possesses two consensus RNA-binding motifs (RNP1 and RNP2) was identified as a universal nucleic acid-binding module that is capable of 
binding to RNA, ssDNA and dsDNA (Landsman, 1992; Manival et al., 2001). In addition, CCHC zinc fingers are also known to bind to ssDNA and RNA (Gorelick et al., 1988; Rajavashisth et al., 1989; Roussell and Bennett, 1993; Dannull et al., 1994; Espinosa et al., 2003). The presence of CSDs and zinc fingers in both OsCSPs suggests that they are functional nucleic acid-binding proteins. The ability to bind to nucleic acids was previously demonstrated for the cold-inducible CSD proteins from winter wheat and Arabidopsis (Karlson et al., 2002; Fusaro et al., 2007; Kim et al., 2007). A systematic deletion analysis of WCSP1 confirmed that the CSD is sufficient for binding to ssDNA and RNA, and that both C-terminal zinc fingers were necessary for dsDNA binding activity (Karlson et al., 2002). In the present study, I employed a gel shift assay using ssM13mp8 DNA to determine whether rice CSD proteins also possess nucleic acidbinding activity. This in vitro assay confirmed that both OsCSP1 and OsCSP2 can bind to ssDNA with apparent non-specificity (Fig. 1-2b). Slight differences in the pattern of the gel shift are likely attributed to the difference in size for the OSCSP1 and OsCSP2 proteins.

To further understand the functions of rice CSD proteins, I utilized a bacterialbased in vivo functional complementation system. The BX04 mutant E. coli strain lacks four CSPs and is impaired for growth under low temperatures. CSD proteins from winter wheat and Arabidopsis can complement this mutant phenotype (Nakaminami et al., 2006; Kim et al., 2007). I confirmed that rice CSD proteins also possess a similar ability to promote growth of the BX04 mutant under low temperature (Fig. 1-3). The complementation assays using plant CSD proteins support the notion that plant CSD proteins may also function as RNA chaperones, similar to bacterial CSPs, during cold stress. Collectively, these in vitro and in vivo functional assays indicated that rice CSD proteins are capable of functioning in a similar fashion to those of winter wheat and Arabidopsis.

To begin to understand the potential role of OsCSPs with respect to lowtemperature stress on a whole-plant level, I treated the rice plants with chilling temperatures $\left(4^{\circ} \mathrm{C}\right)$ and monitored the expression of OsCSPs at both the transcript and protein levels. Both $O s C S P 1$ and $O s C S P 2$ transcripts accumulated marginally in root and shoot tissues during a $24 \mathrm{~h}$ time period. This transient response occurred much more 
rapidly in roots (Fig. 1-4a). The expression profiles for OsCSPs were dramatically different from those previously observed for winter wheat and Arabidopsis. In both of these systems, transcripts steadily increase upon cold treatment over a much longer time course period (Karlson et al., 2002; Karlson and Imai, 2003; Fusaro et al., 2007; Kim et al., 2007).

For our next line of investigation, I evaluated the protein levels of rice CSD proteins subsequent to low-temperature stress and compared these to previously obtained data from winter wheat. I initially performed a dot blot assay to confirm whether the antiWCSP1 polyclonal antibody could detect both OsCSPs. Both rice proteins were detectable with the antibody; however, OsCSP2 was recognized to a lesser extent (Fig. 16). Therefore, western blot data obtained from rice total protein extracts may not reflect the true relative level of OsCSP2. Subsequent to a prolonged low-temperature stress, both CSPs remained at constant levels for the duration of the $10 \mathrm{~d}$ stress treatment (Fig. 1-7). Conversely, in winter wheat, WCSP1 was previously shown to steadily accumulate throughout the duration of the $47 \mathrm{~d}$ cold treatment period (Karlson et al., 2002). Although our aforementioned in vitro and in vivo studies confirmed that rice CSD proteins are capable of functioning similarly to winter wheat and Arabidopsis CSPs, the response of OsCSPs to low-temperature stress, however, is in sharp contrast to both Arabidopsis and winter wheat, which are capable of cold acclimation. Because rice is susceptible to chilling temperatures (Hahn and Walbot, 1989; Kazemitabar et al., 2003), these correlative data support the notion that plant CSD proteins might be involved in the cold acclimation response to low-temperature stress. Future functional studies are warranted and necessary to understand their in planta functional role with respect to lowtemperature stress.

In addition to providing a critical function during low-temperature stress, several bacterial and animal CSD proteins play various important developmental roles. To determine whether rice CSD proteins may play a role in development and if they are differentially expressed in different tissues during various developmental stages, I monitored OsCSP protein and transcript levels throughout the entire length of the rice plant life cycle. Rice CSD proteins showed highest accumulation in developing panicle tissues and were also accumulated in flowers and seeds. From a viewpoint of low- 
temperature stress, this accumulation pattern is interesting because the reproductive stage of rice is most sensitive to low-temperature exposure (Imin et al., 2004). In addition to reproductive tissues, OsCSPs accumulated at higher levels in the tissues with active meristems such as hypocotyls, radicals and crown tissue (Fig. 1-8). These data are also supported by the study where OsCSP2 was identified as one of the 16 highly abundant proteins that are accumulated in the panicle (Dooki et al., 2006). Similar to the expression of rice CSPs, an Arabidopsis CSD protein (AtGRP2; At4g38680) is highly expressed in apical meristems, ovules, embryos and seeds (Fusaro et al., 2007). When AtGRP2 is down-regulated, it results in many developmental abnormalities with respect to flowering time, stamen number and seed development (Fusaro et al., 2007). These data indicate that plant CSD proteins may also play an important role in various aspects of plant development (Fusaro et al., 2007). However, at the present time, a clear understanding for the molecular mode of action for plant CSD proteins has not been elucidated in higher plants.

Several vertebrate Y-box proteins play important roles during development by effecting the translation of certain transcripts through RNA masking. For example, several Y-box proteins like Xenopus FRGY2 and mouse MSY1 and MSY2 form the major components of translationally inactive mRNPs, which are stored throughout gametogenesis (Murray et al., 1991; Gu et al., 1998). The stored mRNAs eventually become translationally active during embryogenesis, which coincides with the massive degradation of stored germinal Y-box proteins (Wolffe et al., 1992; Sommerville, 1999). In vitro RNA-binding studies suggest that $\mathrm{YB}-1$ exerts a dual effect on translation, stimulating it at a low YB-1/mRNA ratio, while completely suppressing it at a higher YB-1/mRNA ratio (Minich and Ovchinnikov, 1992).

A CSD protein from the green alga Chlamydomonas reinhardtii was recently shown to affect the expression of $L H C B M$ mRNA through a mechanism bearing similarity to the RNA masking activity of FRGY2 (Mussgnug et al., 2005). It is therefore reasonable to consider that plant CSPs may function in the stabilization of certain mRNAs during various developmental stages, thereby effecting plant development. In addition, Y-box proteins also act as transcription factors by binding to the Y-box sequence (inverted CCAAT motifs in a special surrounding), which is present in 
promoter and enhancer regions. By binding to such sequences, YB-1 either positively or negatively regulates the transcription of many genes (Swamynathan et al., 1998; Kohno et al., 2003; Skabkin et al., 2006), including some viral genes and growth factor genes, and genes involved in cell division, apoptosis, the immune response, multiple drug resistance, stress responses and regulation of tumor growth (Skabkin et al., 2006). Further studies are necessary to establish the functional role of plant CSD proteins in both plant development and the adaptation to low-temperature stress.

\section{ACKNOWLEDGMENTS}

The authors would like to thank Dr Ryozo Imai for generously providing the anti-WCSP1 polyclonal antibody and for providing Yukihikari rice seeds. The authors would also like to thank Dr Masayori Inouye for kindly providing the BX04 mutant strain and the pINIII and pINIII: CspA positive control vectors. This work was partially supported by a National Science Foundation grant (IBN-0416945) to D.K. West Virginia Agriculture and Forestry Experiment Station Scientific Article No. 3010. 
FIGURES

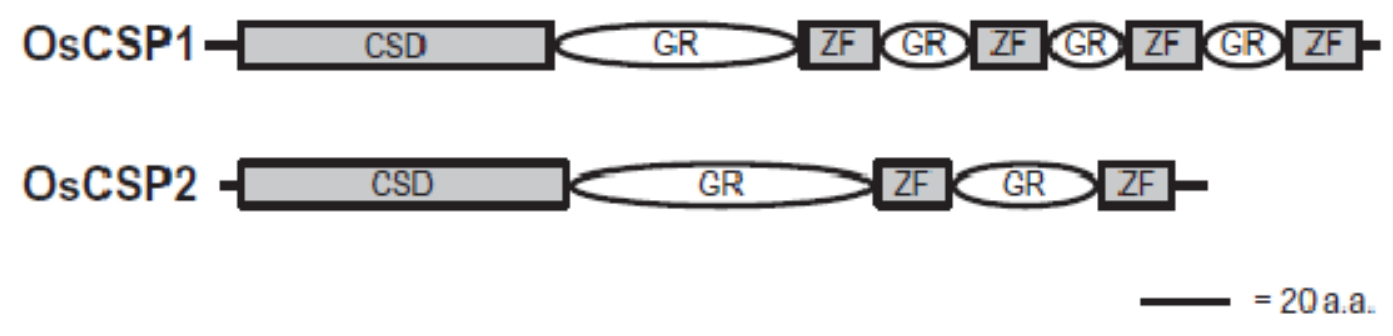

Figure 1-1. Domain architecture for rice CSPs. Both OsCSPs contain an N-terminal CSD and a C-terminal glycine-rich region, which is interspersed with a variable quantity of retroviral-like CCHC-type zinc fingers. GR, glycine-rich region; ZF, zinc finger. 

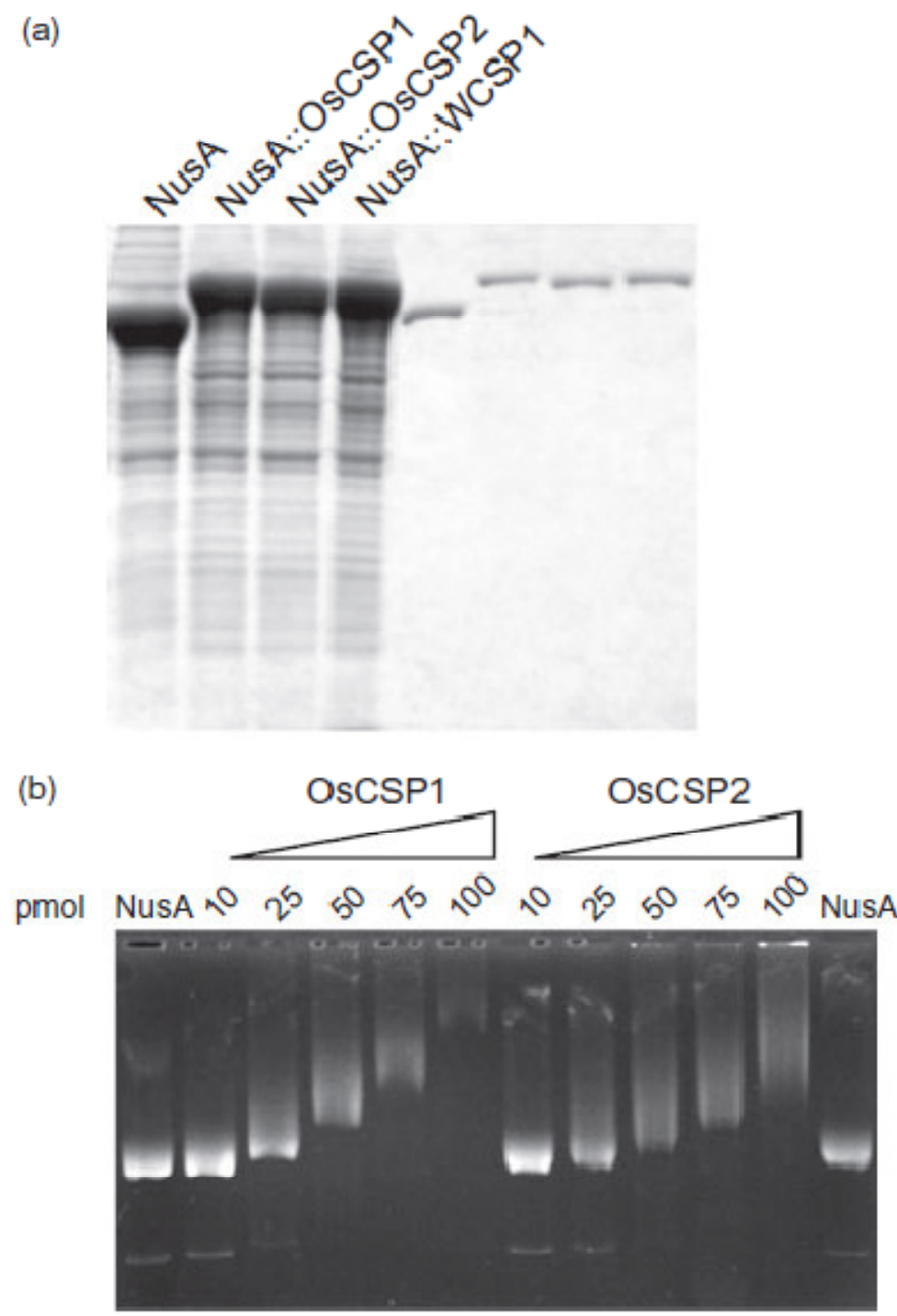

Figure 1-2. ssDNA binding activity of OsCSPs. Recombinant proteins were expressed as $\mathrm{N}$-terminal NusA and C-terminal His tag fusion proteins, and purified from soluble fractions using a Ni-NTA spin column. Affinity-purified fusion proteins were applied to ssDNA binding assays. (a) Sodium dodecyl sulphate-polyacrylamide gel electrophoresis (SDS-PAGE) gel showing the highly induced recombinant proteins in the soluble fractions and the affinity-purified proteins. (b) Nucleic acid-binding assay with M13mp8 ssDNA and purified OsCSP proteins. Increasing concentrations of both OsCSP1 and OsCSP2 resulted in retardation of mobility of ssDNA in the gel. When added at the highest concentration, the negative control NusA protein did not affect the migration of DNA. 


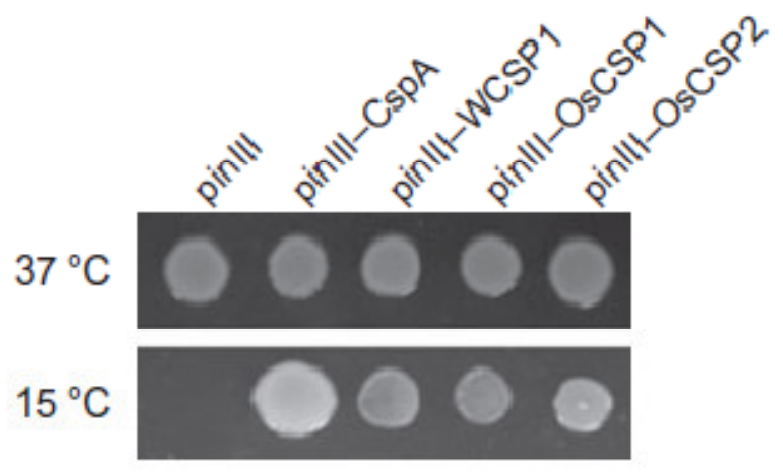

Figure 1-3. Rice CSPs complement cold-sensitive phenotype of bacteria. The BX04 strain of Escherichia coli is a quadruple mutant lacking four endogenous CSPs. Rice and wheat CSPs were cloned into an inducible pINIII vector and transformed into BX04 cells and observed for growth. When grown at $37^{\circ} \mathrm{C}$ overnight, all of the colonies, including the cells transformed with the empty vector, grew normally and formed visible colonies. When the cells were grown at $15^{\circ} \mathrm{C}$, only the cells that were transformed with empty vector failed to show any visible growth after a period of $7 \mathrm{~d}$. 

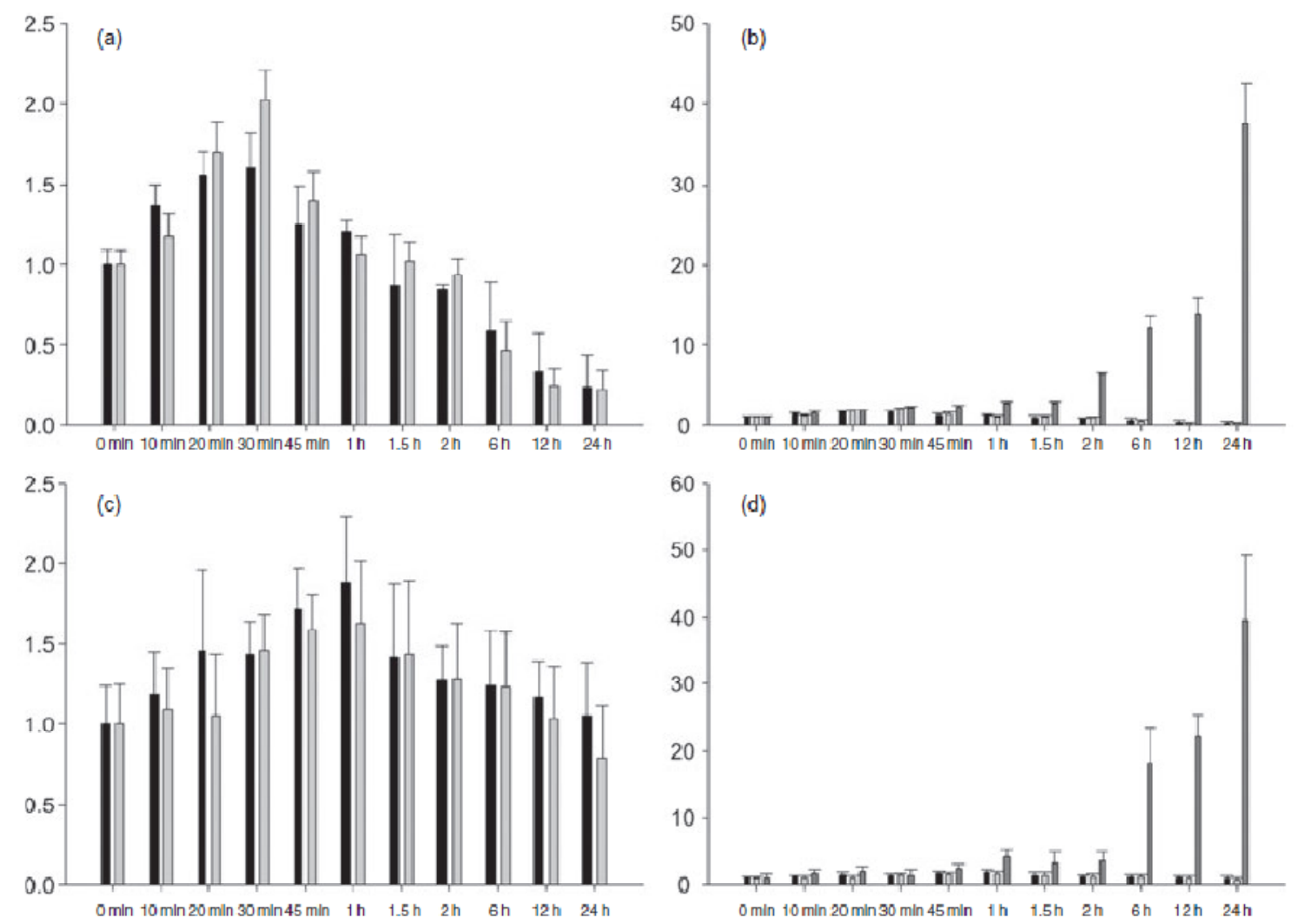

Figure 1-4. Expression of OsCSPs upon treatment with low-temperature stress. qRTPCR was employed to study the expression of OsCSPs, in which the 18SrRNA gene was used as an internal control for normalization. (a) Relative expression levels of OsCSP transcripts in root tissue. Note the transient and marginal increase in mRNA of OsCSPs and eventual down-regulation. (b) Relative expression of OSCSP1 and OSCSP2 genes under low-temperature stress $\left(4^{\circ} \mathrm{C}\right)$ in root tissue along with the positive control low temperature-induced gene (OsLIP19). Note the very low levels of OsCSP transcripts with respect to the abundantly accumulating transcripts of OsLIP19. (c) Relative expression of OsCSPs in the shoot tissue upon cold treatment. Note that the transient and slight increase in transcripts of OSCSPs was delayed when compared to their accumulation in root tissue. (d) Relative expression of OsCSPs in shoot compared to the positive control OsLIP19 gene. Note the very low levels of OsCSP transcripts with respect to the high level of mRNA from OsLIP19. In (a) and (c), black and grey bars represent OsCSP1 and OsCSP2, respectively. In the relative comparisons to OsLIP19 in (b) and (d), OSLIP19 is depicted by dark grey bars. 


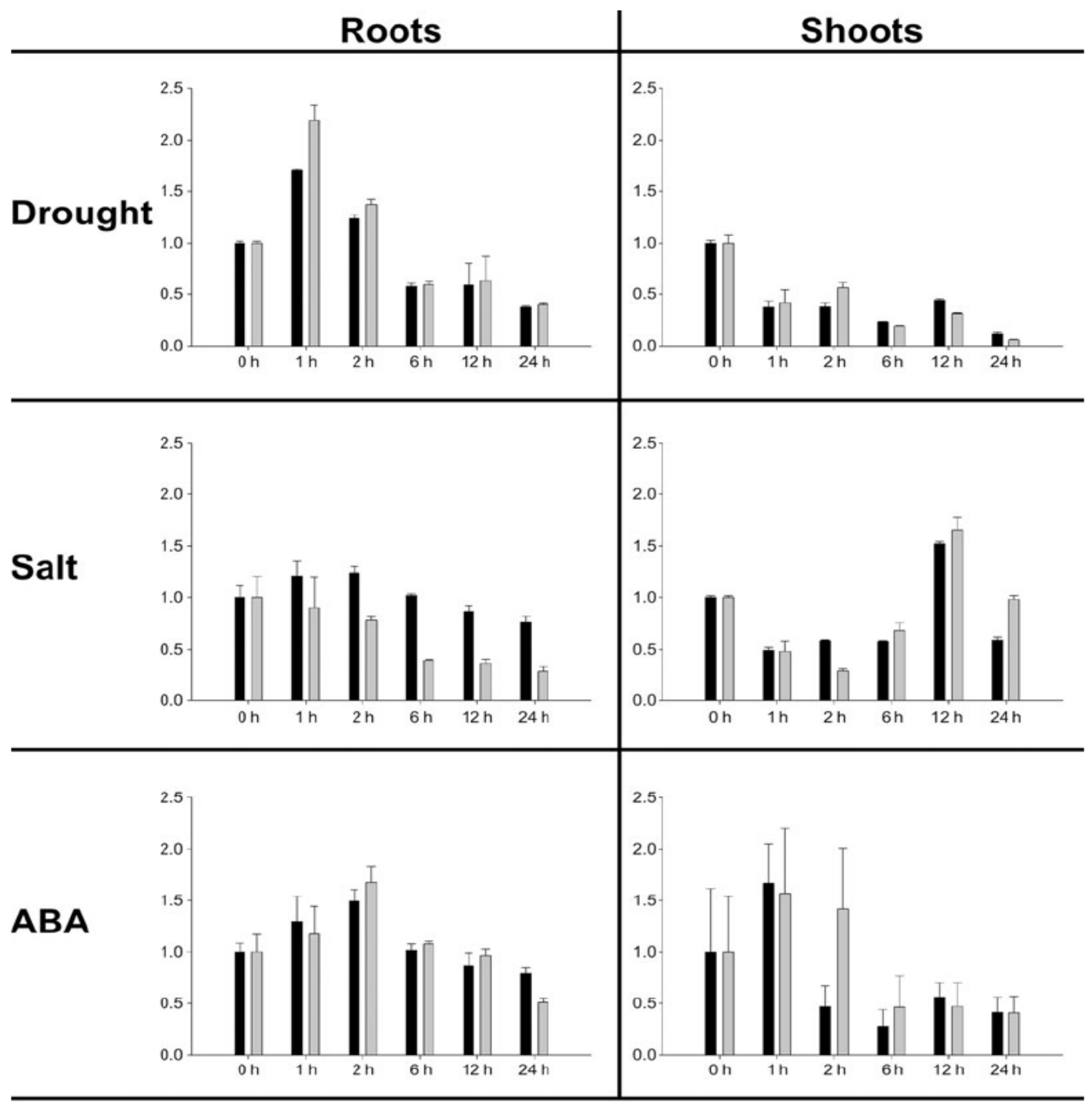

Figure 1-5. Effect of salt, drought and abscisic acid (ABA) stress treatments on the expression of $O s C S P$ transcripts. Rice seedlings were grown for 1 week in a growth chamber at $25^{\circ} \mathrm{C}$ under continuous light in a hydroponic system. Seedlings were removed from water for drought stress, transferred into a $250 \mathrm{mM} \mathrm{NaCl}$ solution for salt stress or transferred into water containing $50 \mu \mathrm{M}$ ABA. A transient increase was noted in the root tissue for both transcripts when treated with drought and ABA. During salt stress, only OsCSP1 mRNA is increased very marginally and transiently in the root tissue. In shoot tissue, with the exception of ABA treatment, no conclusive pattern of transcript expression was noted. ABA treatment resulted in transient increase followed by downregulation of transcripts. 


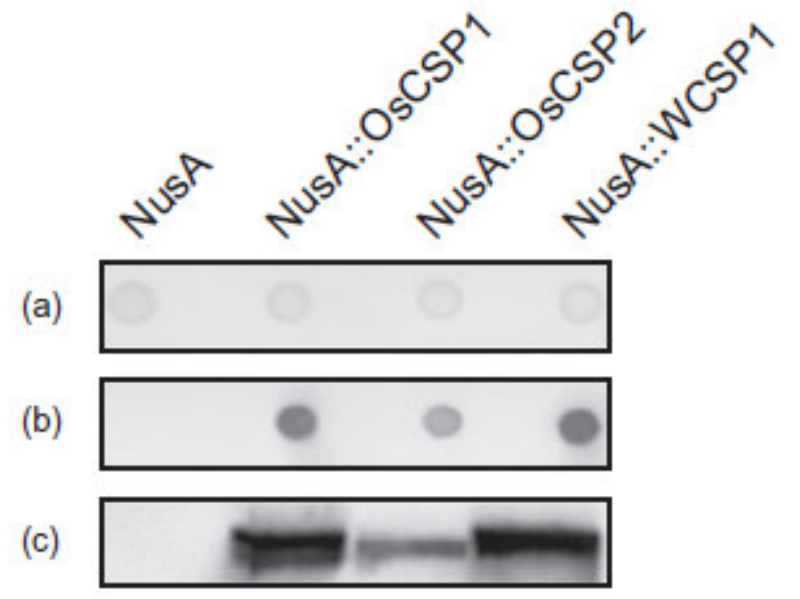

Figure 1-6. Confirmation for the recognition of OsCSPs with the WCSP1 antibody. Recombinant fusion proteins were spotted onto nitrocellulose membrane at different concentrations and subjected to Western blot analysis with anti-WCSP1 polyclonal antibody. (a) Ponceau S-stained membrane to confirm equal loading of recombinant proteins $(250 \mathrm{ng})$. (b) Antibody detection indicating the differential recognition for OsCSP1 and OsCSP2. (c) Western blot detection of sodium dodecyl sulphatepolyacrylamide gel electrophoresis (SDS-PAGE)-separated NusA::OsCSP fusion proteins $(1 \mu \mathrm{g})$. Note that OsCSP1 is recognized at similar levels to that of WCSP1; however, OsCSP2 is recognized at a much lower level. 


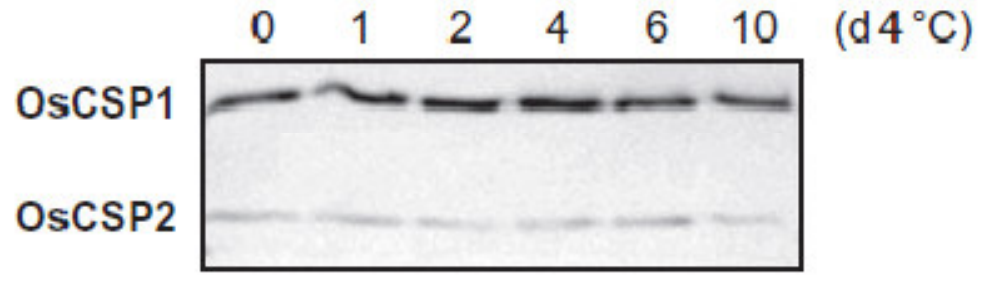

Figure 1-7. Western blot analysis of OsCSP protein expression under low-temperature stress. Rice seedlings were grown for 1 week at $25^{\circ} \mathrm{C}$ under continuous light and were then exposed to prolonged low-temperature stress at $4^{\circ} \mathrm{C}$. Total proteins were isolated from rice crown tissue and subjected to western blotting using the anti-WCSP1 polyclonal antibody. Note that OsCSPs protein do not accumulate during the lowtemperature stress treatment. 


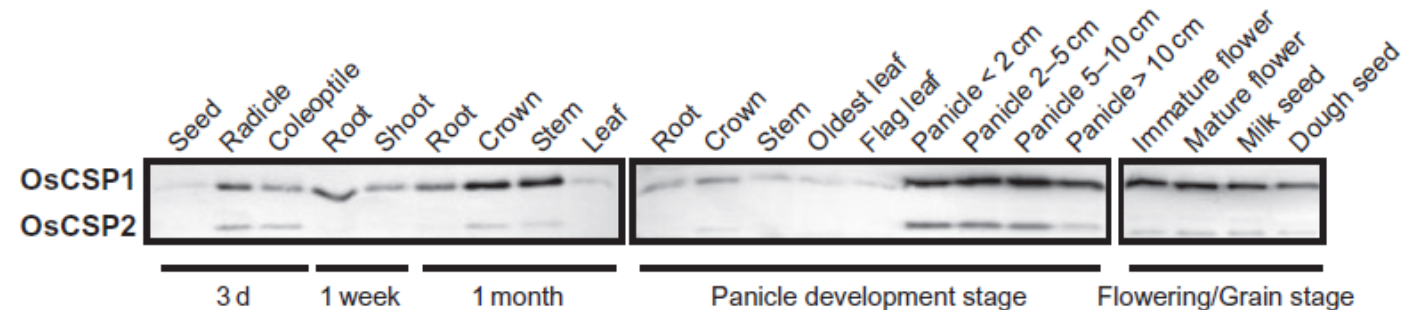

Figure 1-8. Western blot analysis of OsCSP protein expression during rice development. Seeds were germinated in a growth chamber and then grown in a glasshouse until maturity. Tissues were collected at different stages of plant development, and total proteins were isolated and subjected to western blot analysis. Fifteen micrograms of total proteins was separated by sodium dodecyl sulphate-polyacrylamide gel electrophoresis (SDS-PAGE) and transferred to a nitrocellulose membrane for western blot analysis. Note the high accumulation of OsCSPs in reproductive tissues and in hypocotyls, radicles and crown tissues. 


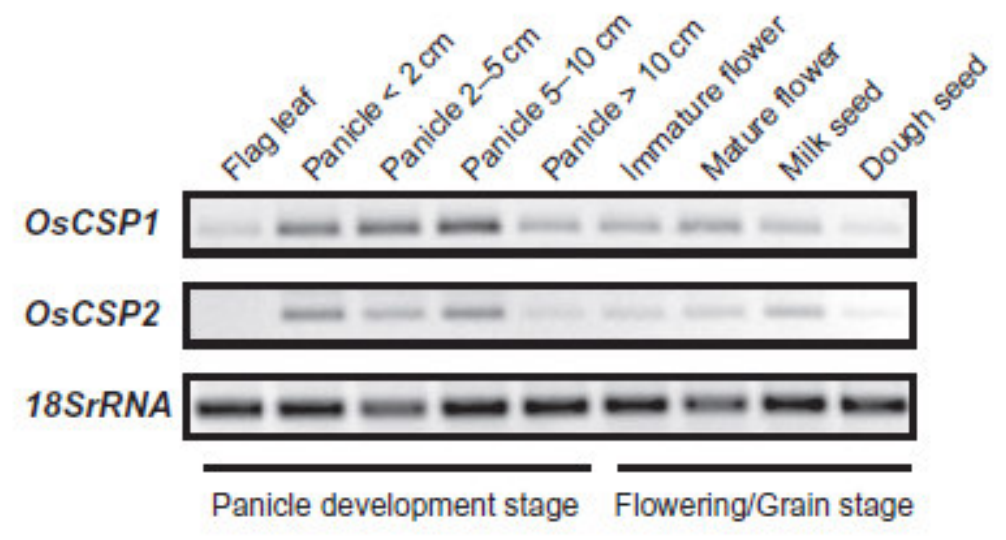

Figure 1-9. Developmental expression patterns of $O s C S P$ transcripts in the reproductive tissues. Semiquantitative RT-PCR was performed on RNA isolated from different tissues obtained during rice reproductive development. Note that when compared to the vegetative flag leaf tissue, $O s C S P$ transcripts are highly expressed in the reproductive tissue including panicles, flowers and seeds. 
Chapter 2: SUMOylation of plant cold shock domain proteins and its effects on protein localization 


\begin{abstract}
In all eukaryotes, SUMOylation is a conserved type of reversible post-translational modification that involves the attachment of SUMO (Small Ubiquitin like Modifier) protein to specific lysine residues on the target protein. In humans and other animal model systems, a huge number of SUMOylation target proteins have been identified and the effects of this modification were characterized for many proteins. In plants, very few target proteins have been characterized as targets for SUMOylation. I determined the presence of SUMOylation motifs in plant cold shock domain proteins by using computational program SUMOplot $^{\mathrm{TM}}$. I experimentally demonstrate that cold shock domain proteins from plants undergo SUMOylation. Using the Arabidopsis cold shock domain protein (AtCSP1), I deciphered the critical lysine residue where SUMOylation occurs by utilizing a mutational approach. I also demonstrate that SUMOylation appears to effect AtCSP1 protein localization.
\end{abstract}




\section{INTRODUCTION}

Small Ubiquitin like Modifier (SUMO) protein can be attached covalently to target proteins in a reversible fashion. The SUMO protein is very similar to ubiquitin in protein size, tertiary structure and in containing a C-terminal diglycine motif (Bayer et al., 1998; Mayer et al., 1998; Melchior, 2000). SUMOylation, the process of attaching SUMO to the target protein, involves a series of similar enzymatic reactions as that of attaching ubiquitin (Melchior, 2000; Verger et al., 2003; Hilgarth et al., 2004; Miura et al., 2007a). Studies on several of the SUMOylation target proteins revealed a consensus SUMOylation motif $\psi \mathrm{KxE} / \mathrm{D}$ (where $\psi$ is a large hydrophobic residue such as Val, Ile, Leu, Met, or Phe, and X is any residue) (Rodriguez et al., 2001; Sampson et al., 2001). Later, it was determined that a stretch of acidic amino acids downstream of the SUMOylation motif aid in SUMO E2 conjugation enzyme attachment, thus expanding the SUMO consensus site to the core $\psi \mathrm{KxE} / \mathrm{D}$ motif and an acidic tail (Yang et al., 2006). A phosphorylation-dependent SUMOylation motif (PDSM), was also observed in some proteins, which is composed of a SUMO consensus site followed by a prolinedirected phosphorylation site (CKXEXXSP), which facilitates cross-talk between the two post-translational modification processes (Hietakangas et al., 2006).

Employing the genetic approaches in model systems like mice, C. elegans, yeast and Drosophila, it was shown that individual SUMOylation components are essential for normal cellular functions and the development of the organism (Melchior, 2000; Zhao, 2007a). At the molecular level, the effects of SUMOylation on target proteins are quiet diverse. These effects were hypothesized to result from changes in protein-protein interactions after SUMOylation (Geiss-Friedlander and Melchior, 2007). The majority of the SUMOylation targets identified were nuclear proteins, indicating essential nuclear functions of SUMOylation (Seeler and Dejean, 2003). Many transcription factors were shown to undergo SUMO modification, which mainly leads to the repression of gene expression (Hilgarth et al., 2004; Johnson, 2004; Gill, 2005; Zhao, 2007a). Repression of gene expression results from enhancement of repression activity of repressors/corepressors and the suppression of activator functions of transcriptional activators/coactivators (Gill, 2005; Lyst and Stancheva, 2007). However, some transcription activators were shown to be activated by SUMOylation, implicating a complex role for 
SUMOylation in transcription (Lyst and Stancheva, 2007; Zhao, 2007a). Recently, it was shown that the building blocks of chromatin, histone proteins, can be SUMOylated which leads to a repression of gene expression (Shiio and Eisenman, 2003; Nathan et al., 2006). In addition, SUMOylation was also shown to affect the DNA binding capability of transcription factors like heat shock proteins (Anckar et al., 2006).

Since the discovery of the first SUMOylation substrate, RanGAP1, SUMOylation was known to effect the localization of proteins. RanGAP1 is a GTPase activating protein for the nucleocytoplasmic transport protein Ran. SUMOylation is required for the localization of Ran to the nuclear pore complex and its interaction with RanBP2 (Matunis et al., 1996; Mahajan et al., 1997; Matunis et al., 1998). In addition, several enzymes of the SUMOylation pathway, such as E3 ligases (like Nup358, RanBP2), E2 conjugation enzyme (Ubc9) and SUMO protease (SENP2) were shown to be associated with the nuclear pore complex implicating that proteins may get SUMOylated as they enter into the nucleus, which may play a role in nuclear retention of these proteins (Matunis et al., 1998; Hang and Dasso, 2002; Zhang et al., 2002; Melchior et al., 2003). SUMOylation of several proteins was shown to be essential for their import into the nucleus (Muller et al., 2001; Pichler and Melchior, 2002; Zhao, 2007a). Within the nucleus, SUMOylation also facilitates protein localization to discrete nuclear structures like PML and TEL nuclear bodies (Sternsdorf et al., 1997; Bloch et al., 1999; Ishov et al., 1999; Zhong et al., 2000; Ishov et al., 2002; Fu et al., 2005). SUMOylation was also implicated in DNA repair as the DNA repair proteins like PCNA, thymidine DNA glycosylase (TDG) and RAD51/RAD52 undergo SUMOylation, which effects their activity (Hoege et al., 2002; Pastushok and Xiao, 2004; Watts, 2006). SUMOylation also plays important roles in maintaining the structure and integrity of the genome (Heun, 2007).

Modification by SUMOylation of a large number of proteins from animal and yeast model systems has been described and the effects of SUMOylation on several of these proteins were deciphered at the molecular level (Seeler and Dejean, 2003; Zhao, 2007a). In plants, very few proteins have been shown to undergo SUMO modification and little is known about the effects of SUMOylation on protein function, localization and stability. The examples of SUMOylation targets in plants include the master regulator of cold stress responses, the ICE1 protein. SUMOylation effects ICE1 protein 
stability and ICE1-dependent transcription (Miura et al., 2007b). SUMOylation of PHR1, a transcription factor that functions in phosphate starvation responses, positively controls the expression of phosphate transporters (Miura et al., 2005). SUMOylation of the flowering regulator, FLD, results in inhibition of its ability to repress expression of FLC by reducing histone acetylation in FLC chromatin (Jin et al., 2007). SUMOylation of another substrate GTE3, a Bromodomain protein, prevented its histone $\mathrm{H} 3$ binding ability (Garcia-Dominguez et al., 2008). Recently, using tag-SUMO-expressing plants, several SUMO target proteins were identified by applying proteomic approaches. The majority of the proteins identified in this study are believed to associate with RNA/DNA dependent and chromatin related processes (Budhiraja et al., 2009). In spite of the limited knowledge about SUMOylation targets in plants, using SUMO pathway mutants, a role of SUMOylation in plant stress responses and development has been deciphered (Novatchkova et al., 2004; Miura et al., 2007a). SUMOylation was implicated in mediating plant responses under abiotic stress conditions like cold (Miura et al., 2007b), salt (Conti et al., 2008b), drought (Catala et al., 2007), heat (Kurepa et al., 2003; Yoo et al., 2006; Saracco et al., 2007) and phosphate starvation (Miura et al., 2005). SUMOylation was also implicated in bacterial and viral pathogenesis (Roden et al., 2004; Lee et al., 2007). In addition, SUMOylation plays essential roles in plant development (Murtas et al., 2003; Catala et al., 2007; Jin et al., 2007; Saracco et al., 2007).

Cold shock domain proteins are ancient universal nucleic acid binding proteins that are conserved from bacteria to animals and plants (Graumann and Marahiel, 1996; Graumann and Marahiel, 1998; Karlson and Imai, 2003). They are primarily implicated in cold stress tolerance in bacteria (Giuliodori et al., 2007; Horn et al., 2007). In higher vertebrates, cold shock domain proteins (referred as Y-Box binding (YB) proteins) function in transcription, translation, and DNA related processes like replication, repair, recombination etc. (Sommerville, 1999; Kohno et al., 2003; Skabkin et al., 2006). Vertebrate and invertebrate cold shock domain proteins were shown to be essential for proper development of the organism (Moss et al., 1997; Yu et al., 2004; Lu et al., 2005, 2006; Uchiumi et al., 2006). In plants, cold shock domain proteins have been implicated in cold stress tolerance and development (Karlson et al., 2002; Karlson and Imai, 2003; Nakaminami et al., 2006; Fusaro et al., 2007; Kim et al., 2007; Sasaki et al., 2007; 
Nakaminami et al., 2009). In vertebrates, the function of cold shock domain proteins has been shown to be regulated by post-translational modifications, such as phosphorylation, and SUMOylation (Sommerville and Ladomery, 1996; Coles et al., 2005; Sutherland et al., 2005; Evdokimova et al., 2006; Bono et al., 2007). In this study, I functionally characterized the SUMOylation motifs in both monocot and dicot cold shock domain proteins for the first time by in vitro assays. In addition to our confirmation of SUMOylation of plant CSPs, I also evaluated the effects of SUMOylation on cold shock domain protein localization.

\section{MATERIALS AND METHODS}

\section{Identification of SUMOylation motifs in cold shock domain proteins}

The sequence for the Arabidopsis cold shock domain protein (AtCSP1) was obtained from TAIR (www.arabidopsis.org). Rice protein sequences (OsCSPs) were obtained from Rice Annotation Project-Data Base (http://rapdb.dna.affrc.go.jp/). Sequences were scanned for SUMO signature motifs by employing the computational program SUMOplot $^{\mathrm{TM}}$ (Abgent, San Diego, CA).

\section{Cloning and Creation of mutations}

For expression in bacteria, coding sequences of $O S C S P 1$ and $O S C S P 2$ were amplified from the constructs as previously described (Chaikam and Karlson, 2008) and cloned into the EcoR1 and Xho1 sites of pGEX-6p-3 (GE Healthcare, Piscataway, NJ) vector . The primers used for the cloning of OSCSP1 were: 5'TCTGAATTCCATGGCGGCGGCGGCGAGGCATCG3' and 5'AGACTCGAGTCACTTGTGGCAGTCGCGGGCGAT3'. For cloning OsCSP2, I used the following primers 5' TCTGAATTCCATGGCGTCGGAGAGGGTGAAGGG3' and 5'AGACTCGAGCTAGTAGGTCTTGCTGGGGCACTC3'. The AtCSP1-pGEX-6p3 construct was described before (Karlson et al., 2009 unpublished results). The lysine residues of the SUMO signature motifs in AtCSP1 protein sequence were converted to arginine by using PCR-based mutagenesis. AtCSP1 K83R and AtCSP1 K194R mutations were created by using the mutational primers 5'TCCTGGTGGTGGTTCTCTCAGGAAGGAGAATAACTCTCGT3' and 
5'AGACCAACGTGGAGCGGTCAGAGGTGGAAACGATGGTTGC3', respectively. The mutations were cloned into a 35s::sGFP(S65T) vector (Chiu et al., 1996; Niwa et al., 1999) by using the forward and reverse primers 5'TCTGTCGACATGGCTTCAGAGGATCAATCGGCGG3',

5'AGACCATGGTAGCTACAGAAGAACATTCCCTT3'. A double mutant construct encompassing the K83R \& K194R mutations was also created using 35s::AtCSP1 K83RsGFP (S65T) plasmid as a PCR substrate and the K194R mutation primer. The mutant versions of the AtCSP1 gene were cloned into the pGEX-6p-3 vector by employing the following primers: 5'TCTGGATCCATGGCTTCAGAGGATCAATC3'and 5'GTACTCGAGTTAAGCTACAGAAGAACATT3'.

\section{Recombinant Protein purification and in vitro SUMOylation assay}

Wild type and variant versions of AtCSP1 recombinant proteins, SUMOylation assays components (expression vectors kindly provided by Dr. H.P Stuible) were prepared as described earlier (Colby et al., 2006; Miura et al., 2007b; Miura et al., 2009). In vitro SUMOylation assays were performed as described earlier (Colby et al., 2006; Miura et al., 2007b; Miura et al., 2009). Immunoblot analysis was performed using anti-GST antibody (Bethyl Laboratories, Montgomery, TX) to detect GST tagged AtCSP1, its variants and SUMOylated products.

\section{Particle bombardment and confocal microscopy}

Onion epidermal peels were bombarded with tungsten particles of size $1.6 \mu \mathrm{m}$ (Bio-Rad, Hercules, CA) using a particle bombardment system according to the manufacturer's instructions (Bio-Rad, Hercules, CA). The cell layers were incubated on MS-agar plates in dark for 20 hours and observed under a Zeiss confocal microscope (Carl Zeiss Micro Imaging Inc. Thornwood, NY).

\section{RESULTS AND DISCUSSION}

\section{SUMOylation motifs in cold shock domain proteins}

Most of the target proteins are SUMOylated on the lysine residue present in the motif $\Psi$ $\mathrm{K}-\mathrm{x}-\mathrm{D} / \mathrm{E}$ (where $\psi$ is a large hydrophobic residue such as Val, Ile, Leu, Met, or Phe and $\mathrm{x}$ 
is any residue) (Rodriguez et al., 2001; Sampson et al., 2001). In addition to the core motif, a stretch of acidic amino acids present downstream enhances the SUMOylation capability (Yang et al., 2006). To determine if plant cold shock domain proteins possess the SUMOylation core motif, I investigated rice and Arabidopsis CSP sequences with the SUMOplot ${ }^{\mathrm{TM}}$ computational program (Table 2-1). SUMOplot ${ }^{\mathrm{TM}}$ predicted that both the rice cold shock domain proteins contain SUMOylation motifs that can be SUMOylated with a high probability (with a score of more than $0.75 / 1$ ). Two SUMOylation motifs containing lysines at positions 40 and 79 were predicted for OsCSP1 protein with scores of 0.94 and 0.76, respectively. The downstream sequence of SUMOylation motif represented at lysine 40 contains two acidic amino acids, however, such residues were absent for the SUMOylation motif represented at lysine79. In addition, the core sequence of the SUMOylation motif at lysine 79 does contain the canonical Glu/Asp residue at the end of the motif, indicating that it may not be an ideal SUMOylation motif. The OsCSP2 protein has only one SUMOylation motif with a high score of 0.91 . This motif contains all of the canonical amino acids along with the downstream acidic amino acids. Among the four Arabidopsis cold shock domain proteins, only the AtCSP1 (At4g36020) protein sequence was predicted to contain SUMOylation motifs with a high probability (score $>0.75$ ) (Table 2-1). The AtCSP1 protein contains two SUMO signature motifs with target lysines placed at amino acid positions at 83 and 194 in core motif sequences LKKE (score-0.91) and VKGG (score-0.76), respectively. However, the motif represented by lysine at 194 position lacks the canonical Glu/Asp residues at the end of core motif, indicating that this motif may not be SUMOylated.

\section{Rice cold shock domain proteins can be SUMOylated}

To determine if rice cold shock domain proteins can be SUMOylated, I performed an in vitro SUMOylation assay on GST fusion recombinant OsCSP1 and OsCSP2 proteins. When highly purified rice cold shock domain proteins (Fig. 2-1a) were incubated with SUMO protein in the presence of SUMOylation pathway enzymes, a higher molecular weight protein band was detected (Fig. 2-1b). This band was not present in mock reactions, which lack SUMOylation enzymes or the rice CSPs, indicating that the high molecular weight band is indeed a SUMOylated form of OsCSPs (Fig. 2-1b). These data 
were in good accordance with the SUMOplot ${ }^{\mathrm{TM}}$ prediction that both rice CSPs can be SUMOylated with high probability.

\section{AtCSP1 protein can be SUMOylated at lysine 83}

To determine if Arabidopsis AtCSP1 protein can undergo SUMOylation, I performed a non radioactive in vitro SUMOylation assay using purified recombinant protein (Fig. 22a). The initial assay with the wild type AtCSP1 protein revealed a higher molecular weight band only in the presence of all the SUMOylation enzymes, indicating that AtCSP1 undergoes SUMOylation (data not shown). Since the SUMOplot program predicted two core motifs with a high probability for SUMOylation, I wanted to determine if both motifs could be SUMOylated. For this purpose, I created mutations in the putative SUMOylation motifs by converting the SUMO acceptor lysines at 83 and 184 positions to arginines. Wild-type and variant proteins were expressed in bacteria as recombinant GST fusion proteins and used for in vitro SUMOylation assays (Fig. 2-2a). When the reaction products were probed with a GST antibody on a western blot, a higher molecular weight band was detected in the reactions containing wild-type and K194R variant proteins. This band was absent in the reactions containing the AtCSP1 (K83R) or AtCSP1 (K83R\& K194R) proteins. Also, this higher molecular weight protein band was not detected in mock reactions lacking AtCSP1 protein or SUMOylation enzymes (Fig. 2-2b). These data indicate that the K83 residue is the critical residue for SUMOylation of the AtCSP1 protein.

\section{Mutation in the SUMOylation sequence appears to alter AtCSP1 protein localization}

One of the major effects of SUMOylation on target proteins is an alteration of protein localization. Hence, I wanted to determine if SUMOylation imparts such changes in the localization of plant cold shock domain proteins. I cloned the wild type (WT) and double mutant (K83R\&K194R) versions of $A t C S P 1$ sequences in frame as a C-terminal fusion to sGFP(S65T), which is driven by the 35s promoter. These constructs were employed for transient expression assays in onion epidermal cells using a particle bombardment system (Fig. 2-3). The onion cells transformed with the 35s::sGFP(S65T) construct showed GFP signal in the nucleus, cytoplasm and the plasma membrane. Cells transformed with the 
35s::AtCSP1(WT)-sGFP(S65T) construct showed GFP signal only in the nucleus and the cell membrane. However, the cells transformed with the 35s:: AtCSP1(K83R\&K194R)sGFP(S65T) construct showed GFP signal in the cytoplasm in addition to the nucleus and the plasma membrane. Based on the in vitro SUMOylation assay and localization experiments, it appears that the inability of AtCSP1 to undergo SUMOylation effects its protein localization. However, from these experiments it is not known if AtCSP1 undergoes SUMOylation in the cytoplasm or in the nucleus or while entering into nucleus.

Considering the wide platform for SUMOylation in different cellular compartments (Geiss-Friedlander and Melchior, 2007), SUMOylation of AtCSP1 might occur in any cellular vicinity. Depending on the location of AtCSP1 SUMOylation in the cell, two scenarios can be hypothesized to explain the alterations in protein localization among the wild type and double mutant AtCSP1 proteins. In the first scenario, it is possible that the SUMOylation of AtCSP1 might affect AtCSP1 protein import into the nucleus. It is also possible that AtCSP1 get SUMOylated in the cytoplasm or while it enters the nucleus. For several proteins like Krüppel-like factor 5 (Du et al., 2008), Daxx (Chen et al., 2006), NFAT1 (Terui et al., 2004), and TEL (Wood et al., 2003) SUMOylation promotes their import into the nucleus. SUMOylation could also promote the nuclear import of wild-type AtCSP1 protein, so that the wild-type protein form primarily exists in the nucleus. In contrast, the double mutant, which cannot undergo SUMOylation, exists in the cytoplasm. However, if the nuclear localization of AtCSP1 protein entirely depends on the SUMOylation, I would anticipate a complete loss of nuclear localization in the double mutant AtCSP1 protein. Since the nuclear localization of double mutant AtCSP1 was not completely lost (Fig. 2-3), there might be other factors regulating the nuclear localization of AtCSP1 in addition to SUMOylation. It is also possible that SUMOylation may enhance the import efficiency of AtCSP1 protein into the nucleus as hypothesized for other SUMOylated proteins (Pichler and Melchior, 2002). This could potentially result in more efficient import of wild type AtCSP1 into the nucleus than the double mutant variant of AtCSP1. In the second scenario, SUMOylation might be occurring in the nucleus. Such nuclear SUMOylation was described for proteins such as p53, Lef1, c-Jun etc., whose modification is stimulated by intranuclear PIAS E3 
ligases (Kahyo et al., 2001; Sachdev et al., 2001; Pichler and Melchior, 2002; Schmidt and Muller, 2002). SUMOylation of AtCSP1 in the nucleus might be influencing the retention of AtCSP1 in the nucleus, which may result from masking of the nuclear export signal or facilitating interaction with other proteins that prevent nuclear export as envisioned for other proteins (Melchior et al., 2003).

In conclusion, I have determined that in silico prediction of SUMOylation with the SUMOplot ${ }^{\mathrm{TM}}$ program can function as an accurate predictor of SUMOylation status for plant cold shock domain proteins. Furthermore, I have incorporated site-directed mutagenesis to precisely confirm that a representative plant CSP is indeed SUMOylated and that this modification affects its sub-cellular localization pattern in planta. 


\begin{tabular}{|l|l|l|l|}
\hline Protein & $\begin{array}{l}\text { SUMOplot } \\
\text { prediction }\end{array}$ & Score & Sequence \\
\hline OsCSP1 & K40 & 0.94 & IKADGFRSLAEGEQ \\
& K79 & 0.76 & VKGGAGGGGGGGGG \\
\hline OsCSP2 & K39 & 0.91 & LKSDGYRSLNDGDV \\
\hline AtCSP1 & K83 & 0.91 & LKKENNSRGNGARR \\
& K194 & 0.76 & VKGGNDGCYTCGDV \\
\hline
\end{tabular}

Table 2-1: SUMOplot ${ }^{\mathrm{TM}}$ prediction of SUMOylation sites in rice and Arabidopsis cold shock domain proteins. The core SUMOylation motif is indicated by red and the SUMOylation enhancing acidic residues are indicated by blue. 


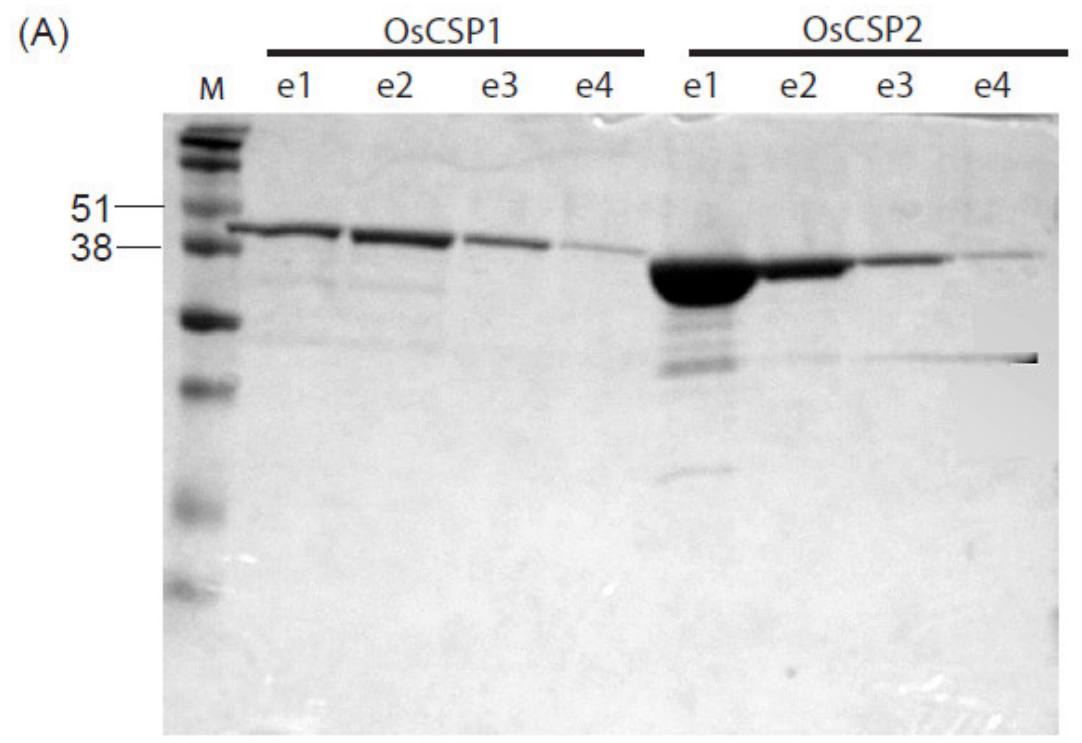

(B)

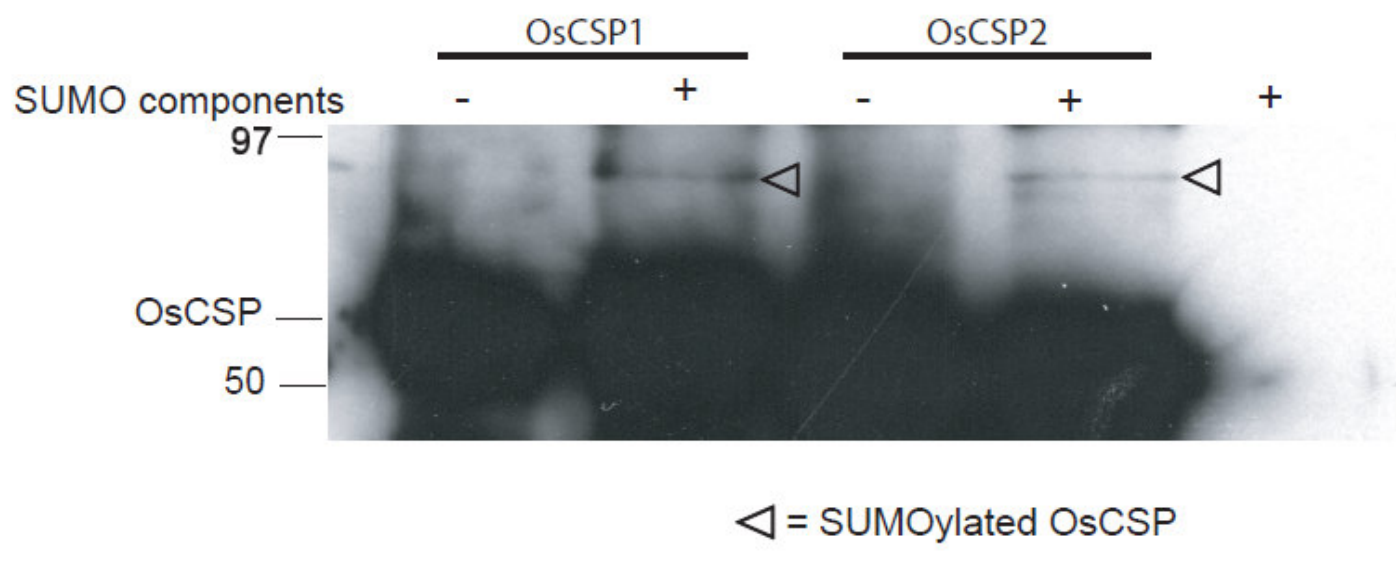

Figure 2-1: In vitro SUMOylation of rice cold shock domain proteins. (a) Purification of bacterially expressed GST-tagged recombinant rice cold shock domain proteins (OsCSPs). The abbreviations e1, e2, e 3 and e4 represent different elutions from the purification column. Note the band of highly purified OsCSP1 and OsCSP2. (b) In vitro SUMOylation assay. Recombinant OsCSPs were incubated with SUMOylation reaction components and the reaction products were subjected to western blot analysis with an anti-GST polyclonal antibody. Protein products were detected by autoradiography. 
(a)

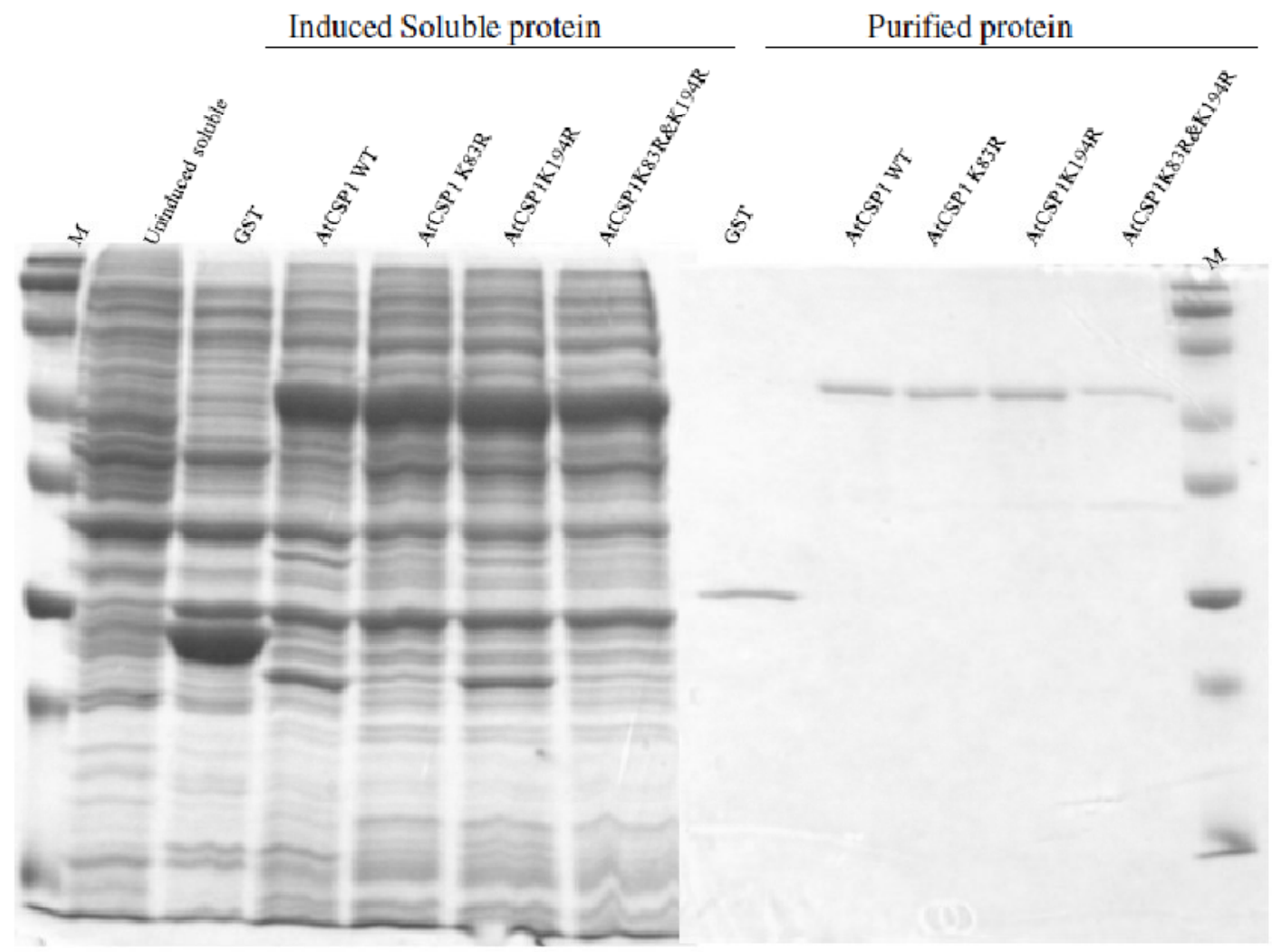

(b)

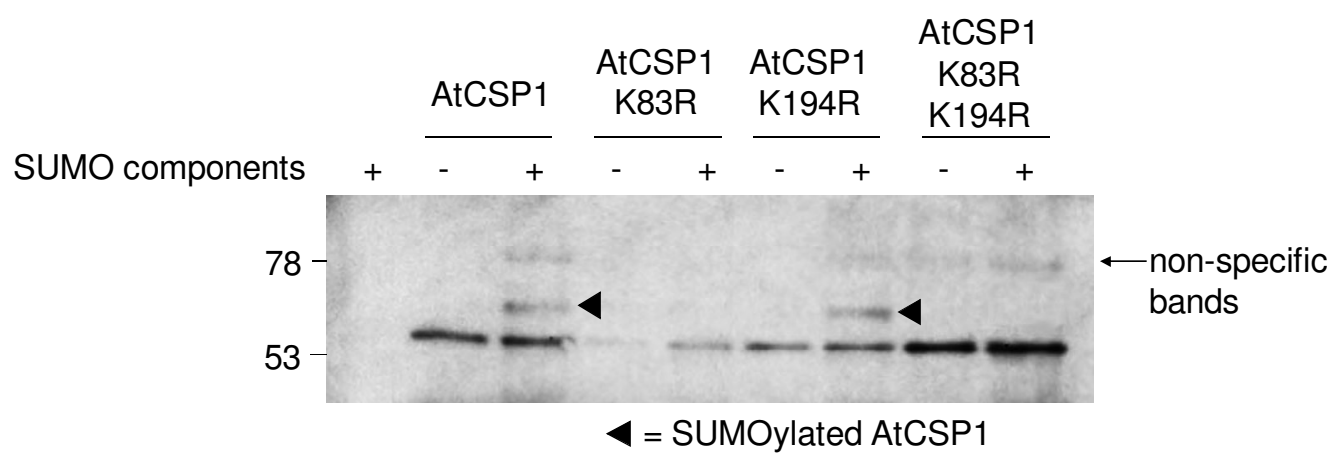

Figure 2-2: Arabidopsis cold shock domain protein AtCSP1 undergoes SUMO modification on lysine 83. (a) Expression and purification of wild-type and mutant versions of AtCSP1 protein. GST-tagged wild-type and mutant versions of AtCSP1 were highly induced in the soluble fraction, and were purified on glutathione sepharose column. (b) in vitro SUMOylation assay. Wild-type and mutant AtCSP1 proteins were incubated with the SUMOylation cycle components. The reaction products were subjected to western blot analysis with a GST-specific antibody. 


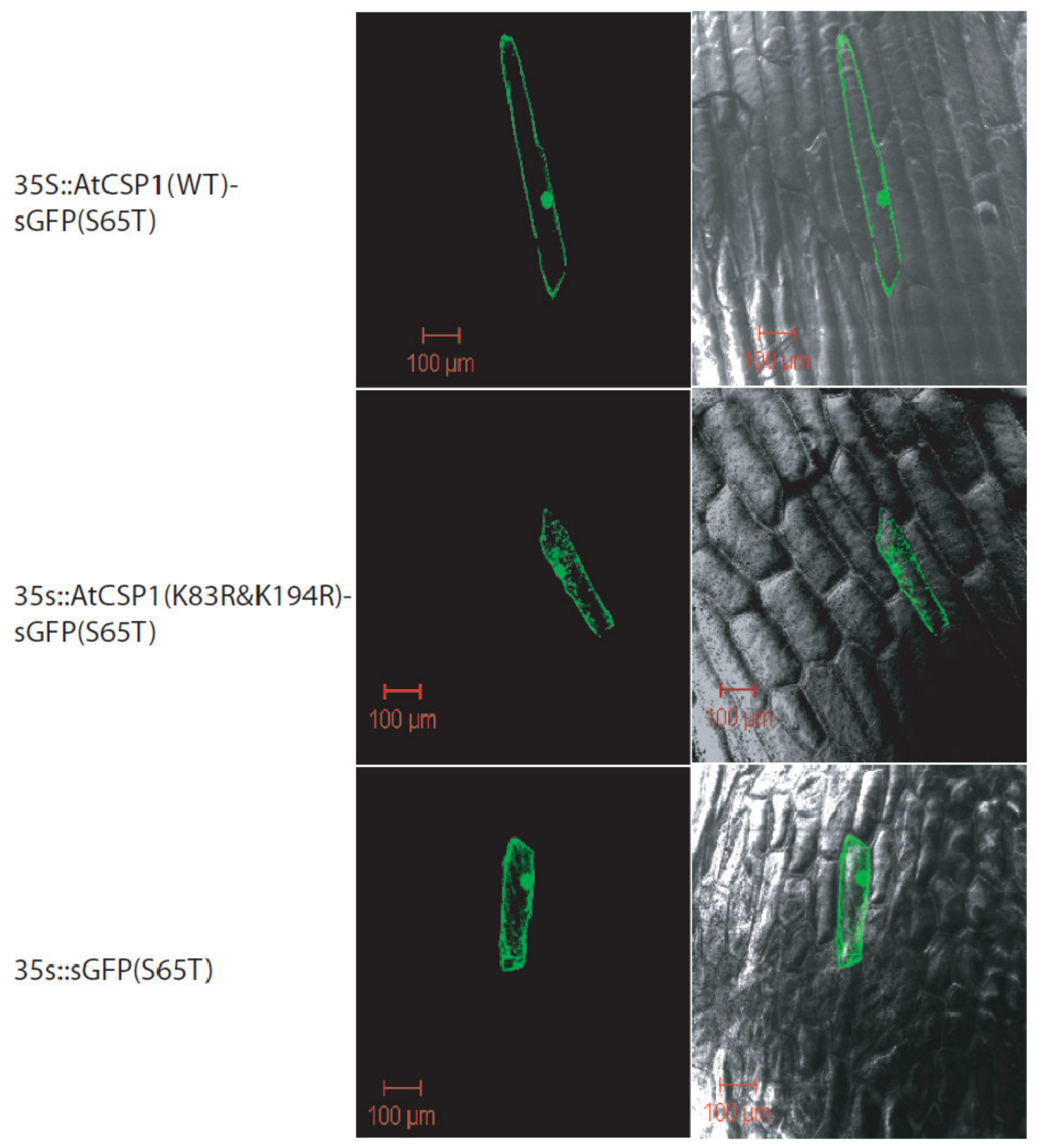

Figure 2-3: Mutation in SUMOylation motifs of AtCSP1 protein effects its localization. Wild-type and double mutant sequences were cloned in frame with C-terminal sGFP(S65T) and are driven by the $35 \mathrm{~S}$ promoter. The constructs were bombarded into onion epidermal cells and visualized with confocal microscopy. The left panel represents the GFP fluorescence images and the right panel represents the GFP fluorescence and DIC merged images. 


\section{Chapter 3: Response and transcriptional regulation of rice SUMOylation system during development and stress conditions}




\begin{abstract}
Modification of proteins by the reversible covalent addition of small ubiquitin like modifier (SUMO) protein has important consequences affecting target protein stability, sub-cellular localization, and protein-protein interactions. SUMOylation involves a cascade of enzymatic reactions which resembles the process of ubiquitination. In Arabidopsis, SUMOylation affects plant development and its ability to survive under a variety of abiotic stress conditions. However, regulation of the SUMOylation system during stress and development is not well understood in plants. In this study, I characterized the SUMOylation system from an important crop plant, rice, and show that it responds to cold, salt and ABA stress conditions on a protein level via the accumulation of SUMOylated proteins. I also characterized the transcriptional regulation of individual SUMOylation cascade components during stress. Majority of the SUMO component genes were decreased in their transcript abundance under stress conditions. However, the transcript abundance of OsSIZ2, an E3 ligase, is increased in response to all the stress conditions, suggesting that it has a role in SUMO conjugate accumulation. During plant development, SUMO conjugates accumulate highly in panicles, developing seeds, crown and stem tissues. At transcriptional level, SUMO component genes are highly expressed in reproductive tissues like young panicles and milk seed. Taken together, these data suggest a role for SUMOylation in rice development and stress responses.
\end{abstract}




\section{INTRODUCTION}

Post-translational modifications of proteins through the reversible covalent attachment of small proteins like ubiquitin and ubiquitin-like modifiers have critical effects on protein stability and biological activities. Small ubiquitin-like modifier (SUMO) proteins possess a similar 3-dimensional structure as that of ubiquitin but only show 18\% identity at the amino acid level and have an additional $\sim 15 \mathrm{~N}$-terminal amino acids. Ubiquitination and SUMOylation of target proteins occurs via a cascade of similar enzymatic reactions including the sequential action of E1 enzymes for SUMO activation, E2 enzymes for conjugation and E3 enzymes for ligation. In spite of the structural and mechanistic similarities, ubiquitination and SUMOylation have very different biological consequences. Ubiquitination mostly associates with the proteasomal degradation of target proteins, whereas SUMOylation has various effects on target protein localization, stability, transcription factor activity, interactions with other proteins, other posttranslational modifications and effects on chromatin structure (Muller et al., 2001; Nathan et al., 2003; Verger et al., 2003; Hilgarth et al., 2004; Miura et al., 2007a; Zhao, 2007b).

All of the SUMOylation system components are present in plant genomes and some have been characterized on a biochemical and genetic level in Arabidopsis (Kurepa et al., 2003; Lois et al., 2003; Novatchkova et al., 2004; Chosed et al., 2006; Colby et al., 2006; Miura et al., 2007a) where they are essential for plant development (Murtas et al., 2003; Catala et al., 2007; Saracco et al., 2007; Jin et al., 2008). During abiotic stress conditions, the SUMOylation system affects plant survival via the accumulation of SUMO conjugates (Kurepa et al., 2003; Downes and Vierstra, 2005; Yoo et al., 2006; Catala et al., 2007; Miura et al., 2007b; Saracco et al., 2007; Conti et al., 2008a). SUMOylation also modulates ABA signaling (Lois et al., 2003), mediates bacterial resistance (Lee et al., 2007) and is involved in viral pathogenesis (Castillo et al., 2004; Roden et al., 2004). Even though the effects of SUMOylation are well understood at a molecular and whole organism level, the regulation of the SUMOylation system in plants remains poorly understood. SUMOylation can be regulated at four levels: (1) by altering the gene expression of SUMO cascade components; (2) by regulating the enzyme activity of proteins involved in SUMOylation; (3) by regulating the recruitment of E3 ligases; 
and, (4) by employing cross talk with other post-translational modifications (Bossis and Melchior, 2006b; Liu and Shuai, 2008). In this study, I report the first comprehensive sequence analysis and transcriptional regulation of all SUMO component genes in the economically important monocot rice model system. I also show that during plant development SUMO conjugates accumulate at higher levels in actively growing tissues and that the SUMOylation system responds to stress conditions via the accumulation of SUMO conjugated proteins.

\section{MATERIALS AND METHODS}

\section{Sequence analysis}

Rice SUMOylation system components have been previously identified (Miura et al., 2007a). In this study, I obtained sequences for individual components from the Rice Annotation Project Database (RAP-DB) (http://rapdb.dna.affrc.go.jp/). Known protein/DNA sequences were used as search queries to BLAST against rice sequence databases to determine if the rice genome encodes additional SUMO, E1, E2 and E3 proteins. I was unable to identify any additional sequences through our database searching. Arabidopsis sequences were obtained from TAIR (http://www.arabidopsis.org), and human and yeast sequences were obtained from the NCBI database. Sequences were aligned using ClustalW (Larkin et al., 2007) and were searched for conserved domains using the Pfam database (Finn et al., 2008). Percentage identity among sequences was determined by application of the Vector NTI® Software (Invitrogen, Carlsbad, CA).

\section{Plant materials and cDNA preparation}

Rice seed sterilization, growth conditions in either growth chambers or glasshouses, stress treatments, RNA isolation and cDNA preparation were performed as previously described (Chaikam and Karlson, 2008).

\section{Real-time PCR analysis}

Primers were designed using 3'UTR sequences for SUMO,E1,E2 and E3 genes to amplify 210 bp regions (Table 3-2). 18SrRNA was employed as a control gene for 
normalization and gene specific primers were designed as previously described (Chaikam and Karlson, 2008). All of the genes were initially amplified using young panicle cDNA as the template with GoTaq ${ }^{\circledR}$ Flexi DNA Polymerase (Promega, Madison, WI) and cloned into pGEMT-easy vector to generate standard curves. Plasmids were quantified using a NanoDropND-1000 (NanoDrop, Wilmington, DE) and the number of copies was calculated based on molecular mass. Absolute standard curves were prepared using SYBR ${ }^{\circledR}$ Green (Applied Biosystems, Foster City, CA) based upon the quantification of plasmid serial dilutions. Absolute quantities were calculated in each sample for $18 \mathrm{SrRNA}$ and all SUMO cascade genes. For the developmental series expression analysis, normalized values $\left(* 10^{6}\right)$ were shown as relative expression. For each stress treatment, fold change was calculated by calibrating the relative expression for each time point with the relative expression of the time zero sample.

\section{Western blots}

Proteins were isolated and quantified as previously described (Chaikam and Karlson, 2008). The only exception was the inclusion of $2 \mathrm{mM}$ N-ethylmaleimide into the SDS loading buffer (Lois et al., 2003) to prevent de-SUMOylation during protein isolation and further storage. For stress treatments, $15 \mu \mathrm{g}$ of total proteins were subjected to SDSPAGE and transferred to nitrocellulose membranes according to standard protocols. For developmental SUMOylation profiles, $25 \mu \mathrm{g}$ of total protein was applied for western blot analysis. Membranes were probed with AtSUMO1 primary antibody (Kurepa et al., 2003) (Abcam, Cambridge, MA) followed by incubation with an HRP conjugated secondary anti-rabbit antibody. Western blots were developed on CL-XPosure X-ray film (Pierce, Rockford, IL) by employing the SuperSignal West Pico chemiluminescent substrate (Pierce,_Rockford, IL).

\section{RESULTS AND DISCUSSION}

\section{Sequence analysis}

All of the SUMO cascade component gene sequences were analyzed by aligning the rice sequences with Arabidopsis, human and yeast sequences. I identified the important 
domains in each set of proteins and documented critical residues. The sequence analysis results were discussed below for individual components.

\section{SUMO genes}

The rice genome encodes three SUMO genes: OsSUMO1 (Os01g0918300), OsSUMO2 (Os01g0918200) and OsSUMO3 (Os07g0574500) (Miura et al., 2007a). On the amino acid level, OsSUMO1 and OsSUMO2 are highly homologous with $89 \%$ identity to each other. They also share more than $80 \%$ identity to AtSUMO1 and AtSUMO2 at the amino acid level (Table 3-1a). OsSUMO3 showed less than $40 \%$ identity with other plant, yeast and human SUMO proteins. In contrast to some Arabidopsis SUMO proteins, which do not have C-terminal diglycine motifs and are believed to be non-functional pseudogenes (Kurepa et al., 2003), the three rice SUMO proteins contain the C-terminal diglycine motif. This C-terminal motif is the site where SUMO proteases can act to produce mature SUMO forms (Fig. 3-1).

\section{SUMO activation enzyme (E1) genes}

The SUMOylation pathway contains two E1 enzymes, named SAE1 and SAE2, which mutually stabilize each other (Boggio et al., 2007) and together form a functional heterodimer. Arabidopsis contains two SAE1 genes (SAE1a, SAE1b), whereas rice contains a single SAE1 gene (OSSAE1a) encoded by locus Os11g0497000 (Miura et al., 2007a). OsSAE1a shows 60\% identity with both Arabidopsis SAE1 proteins (Table 31b). OsSAE1a, which is similar to SAE1 proteins from other organisms, possesses a single N-terminal ThiF domain (Fig. 3-2), which participates in the adenylation of SUMO proteins (Tang et al., 2008)

Similar to other studied organisms, the rice genome contains a single $S A E 2$ gene (OsSAE2; Os07g0586500) (Miura et al., 2007a) with OsSAE2 showing 79\% identity with AtSAE2 at amino acid level (Table 3-1c). Similar to other SAE2 enzymes, OsSAE2 contains three highly conserved domains: an N-terminal ThiF domain containing a GlyX-Gly-X-X-Gly ATP binding domain, followed by an enzyme active site with a catalytic cysteine and a C-terminal ubiquitin fold domain (Fig. 3-3). The catalytic cysteine, where activated SUMO forms a thioester bond, is located at amino acid position 173 in 
OsSAE2. The ThiF domain functions in the adenylation of SUMO protein and the ubiquitin-like domain ( $\mathrm{Ubl}$ ) is important for the interaction and recruitment of E2 enzymes (Tang et al., 2008)

\section{SUMO conjugation enzyme (E2) genes}

Among all of the SUMO cascade components, E2 enzymes are very conserved from yeast to plants and animals with a high level of amino acid identity. Unlike Arabidopsis, which contains a single E2 gene, rice contains three E2 enzymes genes: OsSCE1a, OsSCE1b and OsSCE1c (Miura et al., 2007a). OsSCE1a and OsSCE1b proteins are very closely related to AtSCE1a with $89 \%$ and $86 \%$ identity, respectively. On the other hand, OsSCE1c shows less than $60 \%$ identity with other rice and Arabidopsis E2 proteins (Table 3-1d). OsSCE3 lacks nearly 33 amino acids at its N-terminus and has an extra 11 amino acids at its C-terminus (Fig. 3-4). When studying the alignment of E2 enzymes from plants, yeast and humans, a highly conserved region with an $\alpha \beta \beta \beta \beta \beta(\beta \beta) \alpha \alpha \alpha$ superfold, resembling that of ubiquitin conjugation domain, was observed with a conserved cysteine in the catalytic groove (Tang et al., 2008).

\section{SUMO ligation enzyme (E3) genes}

SUMO E3 ligases facilitate the transfer of SUMO protein onto substrates and play an important role in determining substrate specificity (Weissman, 2001; Palvimo, 2007) and modulate the SUMO attachment to target proteins (Melchior et al., 2003; Hilgarth et al., 2004). Unlike other SUMO components, E3 ligases are more diverse from yeast to animals and plants. Four types of E3 ligases were identified: PIAS (Protein inhibitor of activated STAT), PC2 (Poly comb), RAN-BP (Ran-binding protein) and NSE2/MMS21 (non-SMC element/Methyl methanosulfonate sensitive) (Hilgarth et al., 2004; Hay, 2005; Miura et al., 2007a)). Since only PIAS-type E3 ligases were functionally identified in plants, I focused our sequence and expression analysis on PIAS-type E3 ligases in rice. In contrast to Arabidopsis, which contains a single PIAS-type E3 ligase, two PIAS-type E3 ligase genes were identified in rice (OsSIZ1; Os05g0125000 and OsSIZ2; Os03g0719100) (Miura et al., 2007a). In plant PIAS proteins, three domains can be identified: an N-terminal SAP domain (Scaffold attachment factor-A/B/Acinus/PIAS) 
followed by a plant homeodomain (PhD-finger), and a putative C2HC3 RING-type zinc binding region called a MIZ/SP-ring zinc finger domain (SIZ/PIAS ring) (Fig. 3-5). Plant PIAS-type E3 ligase proteins share a high degree of sequence homology among each other, while exhibiting little sequence homology with those of yeast and human sequences except in the regions of SAP and SP-Ring domains. The SAP-domain is characteristic of many chromatin associated proteins, which is involved in sequence or structure specific DNA binding (Aravind and Koonin, 2000; Okubo et al., 2004; Palvimo, 2007). The C4HC3 PHD finger domain is specific to plant PIAS proteins and is associated with ligase function, as well as interaction with E2 enzymes (GarciaDominguez et al., 2008). The SP-ring domain, which is similar to the RING finger found in RING ubiquitin ligases, lacks two conserved cysteine residues and is important for interaction with E2 enzymes and ligase activity (Hochstrasser, 2001; Hay, 2005). It was recently shown that both PHD finger and SP-ring domains are required for SUMOylation of an Arabidopsis bromodomain protein by AtSIZ1 (Garcia-Dominguez et al., 2008)

\section{Developmental changes in SUMOylation levels and expression of SUMOylation cascade components}

To study the changes in sumoylation profiles during development, I isolated total proteins from various tissues during different rice development stages and subjected them to western blot using a polyclonal antibody developed against the AtSUMO1 protein (Fig. 3-6a). OsSUMO1 and OsSUMO2 proteins show high homology to AtSUMO1 with 89\% and $84 \%$ identity at the amino acid level, respectively. Since the AtSUMO1 antibody was suggested to cross react with the AtSUMO2 protein (Kurepa et al., 2003), which has 87\% amino acid identity with that of AtSUMO1, I hypothesized that the anti-AtSUMO1 antibody could recognize rice SUMO conjugates. Using Arabidopsis crude extracts for western blot analysis, the anti-AtSUMO1 antibody recognized an abundant $14 \mathrm{kDa}$ species in Arabidopsis which likely represents AtSUMO1/2 species (Kurepa et al., 2003). Similarly, the AtSUMO1 antibody recognized an abundant $14 \mathrm{kDa}$ species in rice which likely represents OsSUMO1/OsSUMO2 species (Fig. 3-6a). In addition, high levels of high molecular weight SUMOylated proteins accumulated in large amounts in tissues like crown, stem and panicle which correlate with active growth and development. The lowest 
amount of SUMO conjugates, along with a low amount of free SUMO protein, was observed in leaf and dough seed tissues. In roots, stems and milk seed tissues, moderate amounts of SUMOylated proteins were found. These data indicate that SUMOylation of proteins may have a specialized function in actively growing tissues. These observations are further supported by the proven role of SUMOylation and its components in plant growth and development at a functional level in Arabidopsis (Murtas et al., 2003; Catala et al., 2007; Saracco et al., 2007; Jin et al., 2008).

To understand the role of individual SUMO cascade components and their regulation during development, I studied accumulation of mRNA for individual genes within different tissues by quantitative real-time PCR analysis. In our expression analysis, I were only able to detect OsSUMO1 and OSSUMO2 transcripts among the three SUMO genes tested (Fig. 3-6b). After evaluating multiple primer pairs and using cDNA derived from different tissues, I was still unable to amplify OSSUMO3 (data not shown). In Arabidopsis, even though the genome encodes nine SUMO genes, only four of them are expressed (Kurepa et al., 2003; Saracco et al., 2007). Accumulation of OsSUMO1 mRNA is at least two-fold higher than that of OSSUMO2 in vegetative tissues, however, they are present at comparable levels in reproductive tissues like mature panicles and developing seeds. High levels of mRNA from both of these genes can be found in milk seeds and young panicle tissues. These correlative data suggest that both genes may be functionally important during plant reproductive development. Furthermore, these data also indicate that OsSUMO1 protein has a more generalized function, whereas OsSUMO2 may perform specialized functions during plant reproductive development. OsSUMO2 is expressed at very low levels in leaf tissue, which may contribute to the low levels of free SUMO and SUMOylated proteins detected in leaf tissue (Fig. 3-6a).

Among the E1 genes, mRNA for OsSAEla is present at very high levels compared to OsSAE2 in all of the examined tissues (Fig. 3-6c). A similar observation was noted in Arabidopsis where AtSAEla and AtSAE1b are highly expressed relative to AtSAE2 (Saracco et al., 2007). However, it is interesting to note that the SUMO activation enzymes SAE1 and SAE2 form a functional heterodimer, suggesting that these proteins should be present in equal amounts. AtSAE2 transcript was proposed to be more efficiently translated and/or the protein may be more stable which would result in a 
stoichiometrically equal level for both of the E1 proteins (Saracco et al., 2007). Similar mechanisms may also apply for rice E1 proteins. In rice, both SAE1a and SAE2 transcripts are highly expressed in reproductive tissues like panicles, developing flowers and seeds, indicating a potential role for these genes during reproductive development.

Among the three SUMO conjugation enzyme genes, transcripts for OsSCE1b is present at very high levels (at least 15 fold more) compared to OsSCE1a and OsSCE1c in all of the tissues studied (Fig. 3-6d(i)). All three of the E2 transcripts are specifically enriched in milk seed tissue (Fig. 3-6(d(i) and d(ii)). These results indicate that OsSCE1b is the E2 gene with most abundant transcript and it may function as the major SUMO conjugating enzyme in rice. OsSCEla and OsSCE1c are expressed in all of the tissues at relatively low levels (Fig. 3-6d(ii)).

Among the two PIAS-type E3 ligases, OsSIZ1 mRNA is present at least two-fold higher than that of OsSIZ2 in all of the tested tissues. These correlative data indicate that OsSIZ1 may function as the major SUMO ligase in rice (Fig. 3-6e). mRNA for both OsSIZ1 and OsSIZ2 are highly expressed in panicle and milk seed tissues and OsSIZ2 was specifically enriched in crown tissue. Low accumulation of mRNA of OsSIZ1 and OsSIZ2 was noticed in leaf tissue which may contribute to the lowest levels of SUMOylated proteins which were identified in leaves (Fig. 3-6a).

\section{Response of SUMOylation system to abiotic stress conditions}

To understand the role of SUMOylation in rice stress responses, I examined the changes in SUMOylation profiles after giving cold, salt and ABA stress treatments to rice seedlings over a period of 12 hours. Changes in SUMOylation profiles were subsequently monitored with western blot analysis. To understand the role and regulation of individual components of SUMOylation cascade during stress conditions, I studied the changes in transcript levels with real-time PCR analysis.

\section{(A) Cold stress}

During cold stress at $4^{\circ} \mathrm{C}$, rice root tissues accumulated high molecular weight SUMO conjugates after only 1 hour of treatment. These conjugates were maintained at similar levels during 12 hours of treatment (Fig. 3-7a). This accumulation likely resulted from 
the appearance of new SUMO conjugates or from an increased amount in the levels of SUMOylated proteins that were present prior to initiation of the stress treatments. A similar accumulation of SUMOylated proteins was noted in Arabidopsis upon cold stress, which was mediated to a large extent by the E3 enzyme AtSIZ1 (Miura et al., 2007b). In addition, a master regulator of cold responses in Arabidopsis (ICE1), was identified as a target for SUMOylation. SUMOylation of ICE1 enhances its stability and affects its ability to mediate transcriptional regulation (Miura et al., 2007b). AtSIZ1 T-DNA insertion lines are freezing sensitive and impaired in their capacity to cold acclimate. The accumulation of SUMO conjugates in rice indicates that SUMOylation likely plays an important and similar role in the response to cold stress in this monocot model system.

In response to cold stress, the majority of the SUMO cascade component transcript levels were decreased (Fig. 3-7b). Most prominently, SUMO transcripts and SUMO activation enzyme transcripts were reduced by more than half of their original level after 24 hours of cold treatment. However, SUMO conjugation enzymes OsSCE1b, OsSCE1c and the PIAS-type SUMO ligation enzyme OsSIZ2 showed a transient increase during early periods of cold treatment. These increases in transcript accumulation may contribute to the accumulation of SUMOylated proteins after exposure to cold stress. Given the importance of SUMO ligases in increasing the efficiency of SUMOylation and specificity [30-32], the increase in their transcription may result in the accumulation of ligase proteins and consequently an increase of SUMO conjugated proteins.

\section{(B) Salt stress}

Compared to the rapid changes observed during cold stress, SUMO conjugates gradually accumulated during 12 hours of salt treatment (Fig. 3-8a). This disparity can be attributed to the differences in the physiological perception of these stress treatments under our experimental conditions. The observed increase in SUMOylated proteins may affect plant survival during salt stress. In Arabidopsis, the SUMOylation system was recently shown to respond to salt stress. Double mutants for two SUMO proteases (OTS1 and OTS2) showed extreme sensitivity to salt stress and overexpression of OTS1 increased salt tolerance. These data clearly indicate an important role for the SUMOylation system in response to salt stress (Conti et al., 2008a). Our data are in good accordance and thus 
corroborate the hypothesis that increased accumulation of SUMO conjugates during salt stress may have functional implications in rice.

OSSUMO1 transcripts decreased to a large extent after exposure to salt stress, whereas OSSUMO2 transcripts transiently increased and were maintained nearly at initial levels (Fig. 3-8b). Both SUMO E1 enzyme transcripts are decreased in response to salt stress. Among the E2 enzymes, OsSCEla transcript levels were maintained at similar levels, whereas $O S S C E 1 b$ and $O S S C E 1 c$ showed a significant reduction relative to initial transcript levels. Among the E3 genes, OsSIZ1 showed a transient increase in mRNA, whereas, OsSIZ2 mRNA began to increase in abundance after 6 hours of treatment. Comparative analysis of SUMO conjugate profiles and real-time PCR data enabled us to conclude that OsSIZ2 may play an important role in mediating SUMO conjugate accumulation during salt stress in rice.

\section{(C) ABA treatment}

Abscisic acid mediates plant responses to stress conditions such as cold, salinity and drought (Xiong et al., 2002; Mahajan and Tuteja, 2005). In Arabidopsis, research on transgenic plants overexpressing AtSUMO1 and AtSUMO2 along with co-suppression lines of AtSCE1a, showed that ABA plays a dual role in ABA signaling by positively effecting the induction of $\mathrm{ABA}$ responsive genes and by attenuating the $\mathrm{ABA}$ signaling pathway that leads to growth inhibition (Lois et al., 2003). However, it is not known if ABA mediates these physiological and molecular responses by affecting the SUMO conjugate levels of proteins in plants. Here, I show that ABA treatment effects SUMO conjugate accumulation in rice (Fig. 3-9a). Changes in profiles were reflected after 1 hour of ABA treatment by the appearance of new SUMOylated proteins. These data further support the hypothesis that the SUMOylation of proteins in rice is a functionally important component in the response of rice to abiotic stress.

After treatment with ABA, the majority of SUMO cascade component transcripts exhibited a transient decrease during the first 2 hours of treatment and subsequently returned to basal levels (Fig. 3-9b). The OsSUMO2 gene showed a prominent positive response 6 hours subsequent to ABA treatment, where its transcript accumulation was increased six-fold. Conversely, OsSUMO1 was not induced, thereby indicating a specific 
role for OsSUMO2 in ABA stress response. Among the E1 genes, OsSAEla transcript levels increased after 6 hours and E2 genes show a transient decrease followed by restoration to basal levels. Among the E3 ligases, OsSIZ2 mRNA levels increased 2-fold in 24 hours. These transcript analyses indicate that OsSIZ2 may be responsible for increased SUMOylation levels during $\mathrm{ABA}$ treatment and that the majority of rice proteins may be conjugated by OsSUMO2.

In summary, I demonstrated that rice responds to different abiotic stress treatments by accumulating SUMO conjugated proteins. This study also indicated that individual components of the SUMOylation system are regulated at transcriptional level during stress conditions. Studies on the expression of individual SUMO components and accumulation of SUMO conjugates in different tissues indicated that SUMOylation may play an important role in plant growth and reproductive development in rice.

\section{ACKNOWLEDGEMENTS}

The authors would like to thank Dr. Kenji Miura and Dr. Jed Doelling for helpful discussions. The authors also thank Dr. Jianbo Yao and his lab for assistance in western blot analysis. 


\section{TABLES AND FIGURES}

(A)

\begin{tabular}{|c|c|c|c|c|c|c|c|c|c|c|c|}
\hline 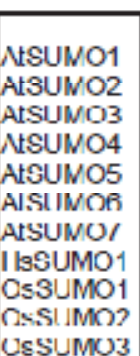 & AtSUMO' & $\begin{array}{c}\text { AtSuIMOZ } \\
97\end{array}$ & $\begin{array}{c}\text { AtSUMO3 } \\
47 \\
45\end{array}$ & \begin{tabular}{|c|} 
ATSUMO4 \\
$4 D$ \\
$4 D$ \\
34
\end{tabular} & $\begin{array}{c}\text { AtSUIMO5 } \\
35 \\
37 \\
35 \\
25\end{array}$ & $\begin{array}{c}\text { AtSUMOE } \\
36 \\
35 \\
31 \\
70 \\
22\end{array}$ & \begin{tabular}{|c} 
A1SUMOT \\
43 \\
39 \\
39 \\
43 \\
35 \\
3 R
\end{tabular} & \begin{tabular}{|c|} 
AsSUMO1 \\
45 \\
45 \\
35 \\
32 \\
31 \\
79 \\
33
\end{tabular} & $\begin{array}{c}\text { OSSUMO1 } \\
80 \\
86 \\
49 \\
41 \\
32 \\
37 \\
11 \\
43\end{array}$ & \begin{tabular}{|c|c} 
OsSuMO2 \\
84 \\
83 \\
46 \\
38 \\
36 \\
36 \\
12 \\
40 \\
89
\end{tabular} & $\begin{array}{c}\text { OsSUMO3 } \\
40 \\
39 \\
33 \\
27 \\
20 \\
23 \\
13 \\
31 \\
38 \\
36\end{array}$ \\
\hline
\end{tabular}

(B)

\begin{tabular}{|c|c|c|c|c|c|}
\hline \multirow{6}{*}{$\begin{array}{l} \\
\text { AtSAF1a } \\
\text { AtSAL1b } \\
\text { HsSAE1a } \\
\text { OSSAE'la } \\
\text { ScAnS1p }\end{array}$} & \multirow[t]{6}{*}{ 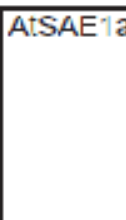 } & \multirow{2}{*}{$\begin{array}{c}\text { AtSAE } 1 \mathrm{~b} \\
81\end{array}$} & HSSAE $1 a$ & & $5 \mathrm{cAOS} 1 \mathrm{p}$ \\
\hline & & & 35 & 60 & 27 \\
\hline & & & 36 & 60 & 27 \\
\hline & & & & 34 & 30 \\
\hline & & & & & 26 \\
\hline & & & & & \\
\hline
\end{tabular}

(c)

\begin{tabular}{|l|c|c|c|c|}
\hline & AtSAE2 & HsUBA2 & OsSAE2 & ScSAE2 \\
AtSAF2 & & 39 & 79 & 31 \\
HsUBA2 & & & 46 & 33 \\
OsSAE2 & & & & 40 \\
SCSAE2 & & & & \\
\hline
\end{tabular}

(D)

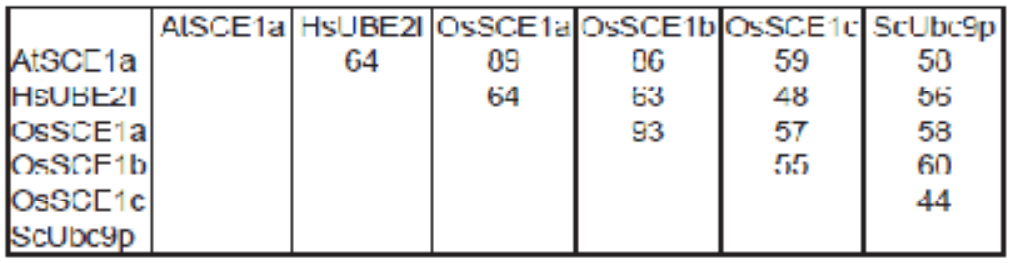

Table 3-1. Amino acid identities among each group of SUMO component genes from different organisms were calculated by using the Vector NTI program. Amino acid identities between different (A) SUMO proteins, (B) SAE1 proteins, (C) SAE2 proteins and (D) SCE proteins were represented in the corresponding Tables. 


\begin{tabular}{|l|l|}
\hline \multicolumn{1}{|c|}{ Gene } & \multicolumn{1}{|c|}{ Primer } \\
\hline OsSUMO1-5' & 5'GGAGTTCTTGCAGCCTCGTA3' \\
\hline OsSUMO1-3' & 5'CACCAGCAACGATGGACATA3' \\
\hline OsSUMO2-5' & 5'AAGACGGCGACGAGATTGA3' \\
\hline OsSUMO2-3' & 5'AACAAAGCCACCCTAATGTCC3' \\
\hline OsSUMO3-1-5' & 5'ACATGGAGGACGGTGATGA3' \\
\hline OsSUMO3-1-3' & 5'GTCGCATCTGCTTAACACCA3' \\
\hline OsSUMO3-2-5' & 5'AGAGTACGTCACGCTGAAGG3' \\
\hline OsSUMO3-2-3' & 5'CTCATCACCGTCCTCCATGT3' \\
\hline OsSAE1a-5' & 5'TTAACTGCTCGACTCCCATTC3' \\
\hline OsSAE1a-3' & 5'GCACAAAACCTTTCCTGGTT3' \\
\hline OsSAE2-5' & 5'GCGGTTGACTTCCGCTATTA3' \\
\hline OsSAE2-3' & 5'TGCAGCCTTACAGCTGACTG3' \\
\hline OsSCE1a-5' & 5'TGCAGCCTTACAGCTGACTG3' \\
\hline OsSCE1a-3' & 5'TTTCACCTCTGTATAGCCGACA3' \\
\hline OsSCE1b-5' & 5'CTACCCGATTCATCATGCAC3' \\
\hline OsSCE1b-3' & 5'CATCACTCAAGCGGTTTCAA3' \\
\hline OsSCE1c-5' & 5'CATGGAGGAGTACAGGAAACG3' \\
\hline OsSCE1c-3' & 5'TTCCAACAGACACATCACCA3' \\
\hline OsSIZ1-5' & 5'GGCGACTGGAGCAGTTTGTA3' ' \\
\hline OsSIZ1-3 & 5'CCGCCACTTCCAGGAATAAT3' \\
\hline OsSIZ2-5' & 5'GAGCTATGGTGGATGGATTGA3' \\
\hline OsSIZ2-3' & 5'CAGGCAACAGAAAAGCATCA3' \\
\hline
\end{tabular}

Table 3-2: Primers used for quantitative real time PCR (qRT-PCR). 


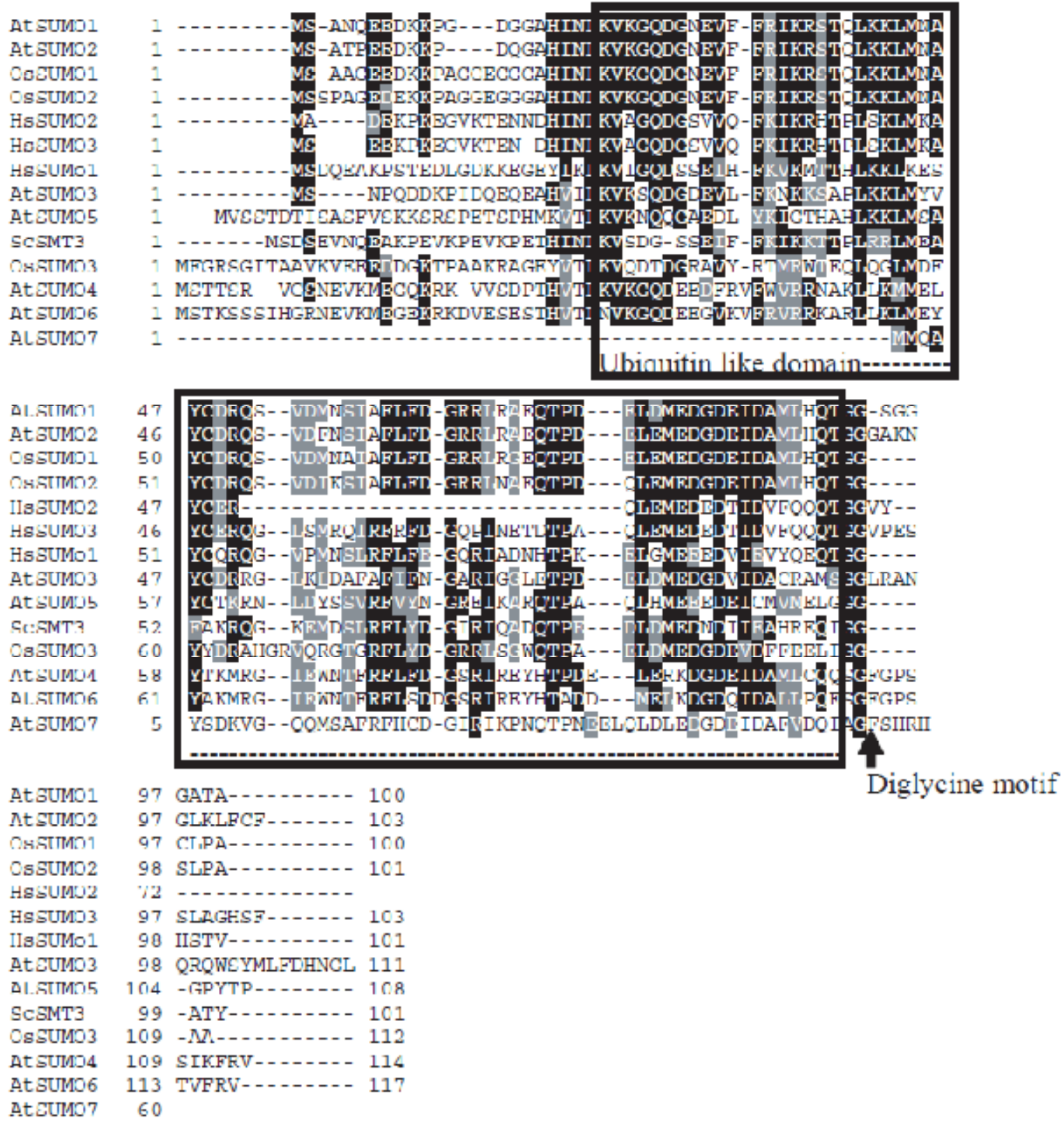

Figure 3-1. Alignment of rice SUMO protein sequences with those of Arabidopsis, yeast and humans. The conserved ubiquitin-like domain and the diglycine motif were depicted. 


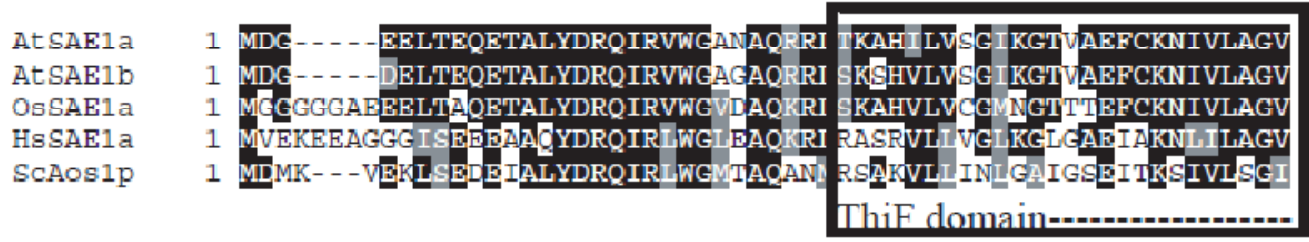

At SAEla

At SAE1b

56 GSVTLMDDR ANME LNANFLIPPDENVŸSGKIVAEI CSDSLKDFNPMVRVSVEKGD

OsSAE1a

56

GSVTLLDDRLVTTEVFNANFLILPDENAYVEKIVAE I C CDSLKDFNPMVHVS IEKGD

Hs SAE1 a

GSLSL DDD L LVTEDDLNANFLI PHDES I YGGRSR AEVCCESLKDFNPMVRVAVEKGD

ScAosip

KGLT LDHEQVIPED PGA OFLIRTG - - SVERNRAEASLERAQNLNDMVDVKVDTED

8 GHLTLDGHMVIEEDIGSOFFIGSED - - VGQWKIDATKERIODINPRIELNFDED

At SAE1a

At SAE1b

OsSAEla

HsSAE1a

ScAosip

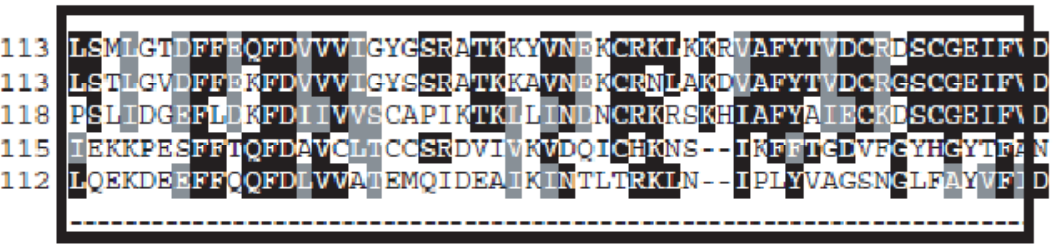

At SAE1a

At SAE1b

Os SAE1a

169 LODYKY TKKKLEEMVECE-

169 LQNYKYTKKKLDETVECE

SCAOS 169 LGEHE EVEKTKVAKVSQG--

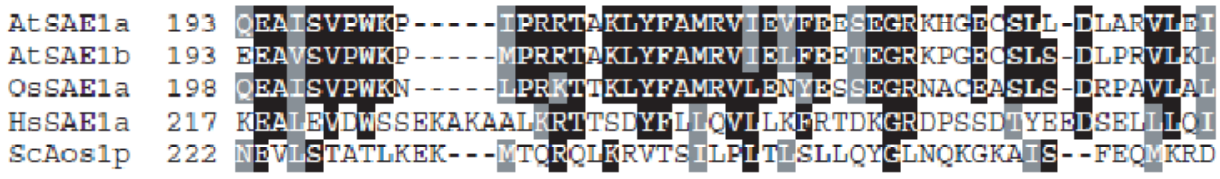

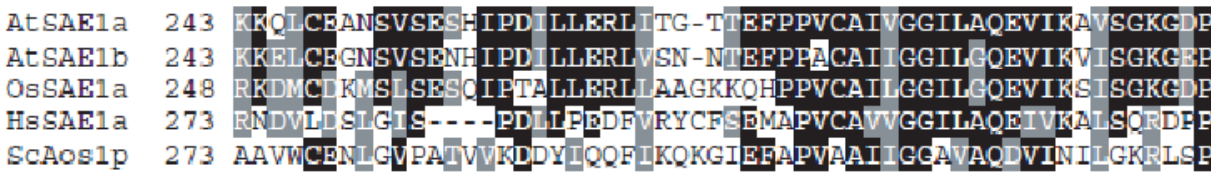

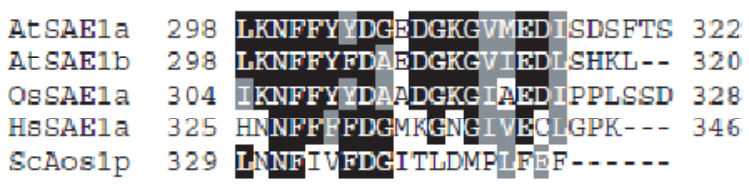

Figure 3-2. Alignment of SUMO E1 enzyme sequences belonging to the SAE1 group. Rice sequences were aligned with those of Arabidopsis, yeast and humans. The conserved ThiF domain is denoted by brackets. 


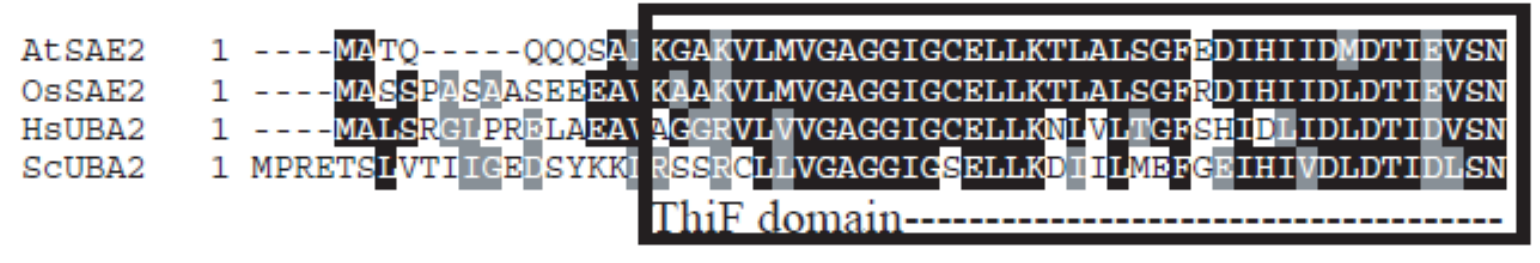

At SAE2

OsSAE2

HsUBA2

SCUBA2

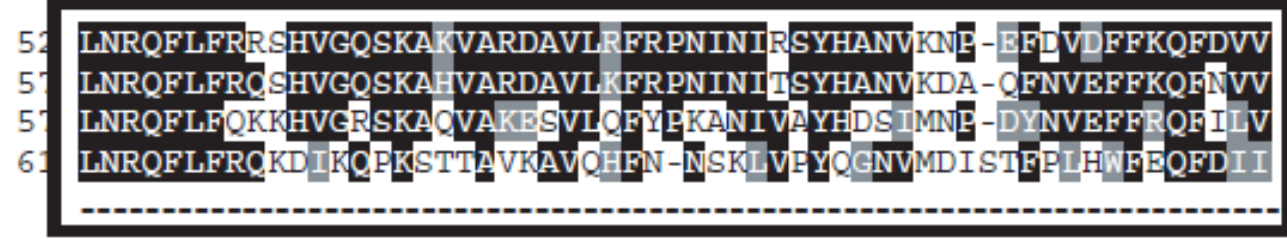

At SAE2

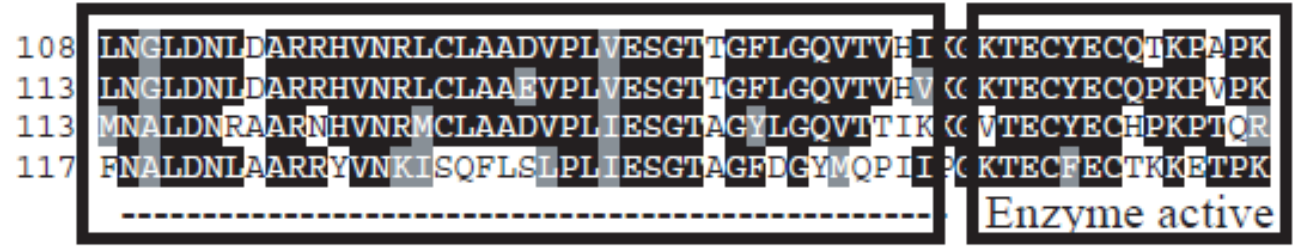

At SAE2 164 TYPVCTITSTPMKFVHCIVWAKDLLFAKLFGDKNQ JNDLN-VRSNNSASSSKETED OsSAE2 HsUBA2 SCUBA2 169 SYPVCTITSTPSKF VHCIVWAKELLFAKMFGDKNQ JNDLN-VRSNESGTS- - -KSD 169 TFPGCTIRNTPSEPIHCIVWAK-YLFNQLFGEEDA JOEVSPDRADPEA AWEPTEA 173 TFPVCTIRSTPSQPIHCIVWAKNFLENQLFASETS BNEDD- -NNQDWG TDDAEEIK

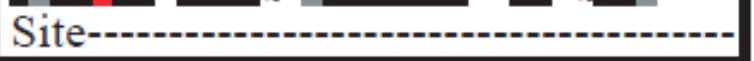
IEQYGRKIYDHVFGSN IEADLSNEETWKNRRRP 221 VFER NADED - - - - - LDQYAR - - - - -RIYDHVFGYN IEVALENEETWKNRRRP 224 ARARASNED GDIKRISTKEWAKSTGYDPVKLFTKLFKDD IRYLLMMDKLWRKRKP SCUBA2 227 RIKQEINELYELQKI I ISRDASRI P - - - EILNKL IQD INKLI AIENLWKTRTKP

At SAE2 OsSAE2 HsUBA2 SCUBA2

At SAE2 OsSAE2 HsUBA2 SCUBA2
261 FPIY STDVLPESITCQNC \$TCNCSV̈TDGDLMVSAM PSLGLKNPQELWGLIDNSIVF 263 NPIYIRDTLPEEAIRQNC SSR--DINNEOEEPSAMVSLGLRNPQEIWSLADNSRVF 280 VPIDWAEVQSQGEETNA Ddd - - -NERCLGIKDQQVLDVKSYARLFSKS- - - - -

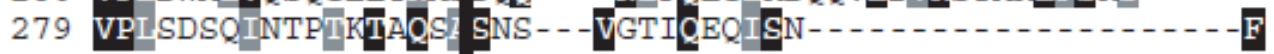
Ubiquitin fold domain

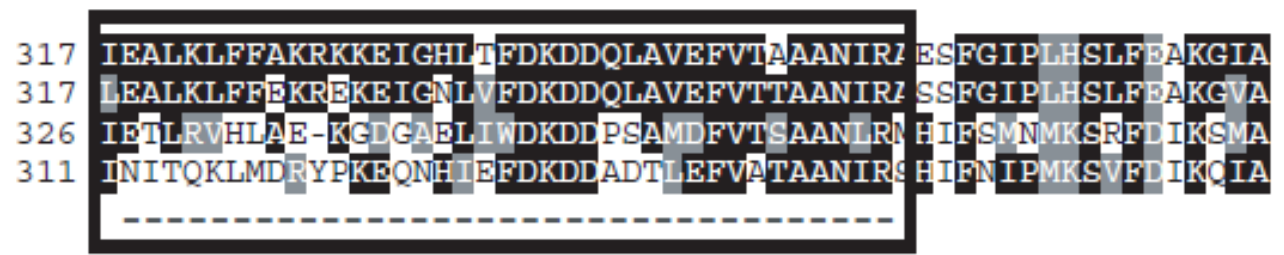

(Continued) 


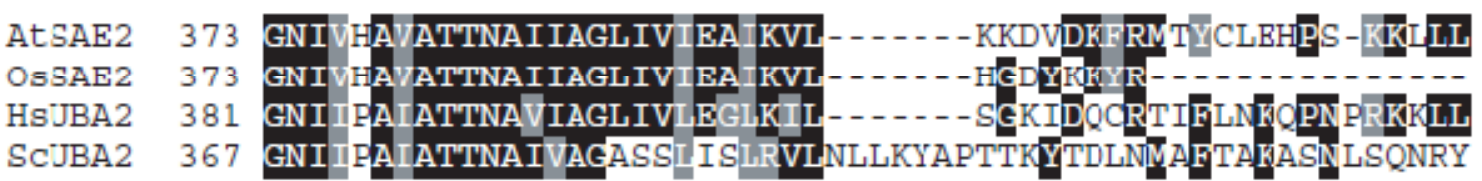

At SAE2

OsSAE2

HsJBA2

SCJBA2

At SAE2

OsSAE2

HsJBA2

ScJBA2

AtsAE2

OsSAE2

$\mathrm{HsJB}$. 2

ScJBA2

AtsAE2

OsSAE2

HsJBA2

ScJBA2

At SAE2

OsSAE2

HsJBA2

ScJBA2
421 MF-IEPY EPNPACYVCSETE-LVLEINTRKSKLRDLVDKIVKTKLGMNLLIMHGN 407 -

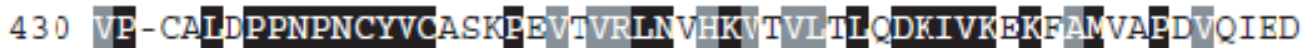
423 ISNPKLAPPNKNOFVCSKVCRGVIKISSDCINKMILSDFVVLIREKYSYE- - - -Q

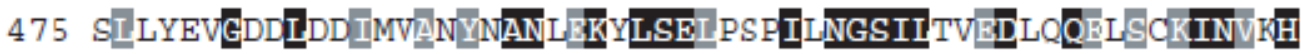
485 - - - -GKGIILISSEEGETEANNHKKLSEFG - -IRNGSRIQADDFLQIYTLINILH 474 DISLLDASNQRLIFDYDFEDLNDRTLSEIN- - IGNGSIILF SDEEGITMIRKAIEL

587 TMFETGRRLTHPLLILFCHREEFDEEKEPEGMVLSGWTPSPATNG트료 STSNNENP 571 $567 \ldots \ldots$ KGADVVAIINSHGKDGIVILDDDEGEIIIDAEP

643 VDVTESSSGSEPASIKRRLSEIEASNHKKETENVESEDDDIMEVENPMMVSKKKIR

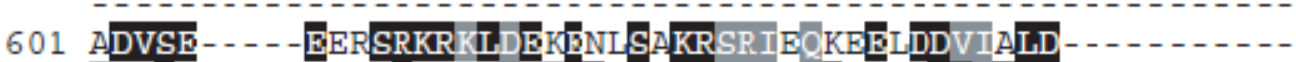
600 INGSK - . - - - KRPVDTEI SEAPSN KRTKIVNEPTNSDI VELD -

$\begin{array}{lll}\text { AtSAE2 } & 699 & \mathrm{VE} \\ \text { OsSAE2 } & & \\ \text { HsJBA2 } & 641 & -- \\ \text { ScJBA2 } & 637 & --\end{array}$

Figure 3-3. Alignment of SUMO E1 enzyme sequences belonging to the SAE2 group. Rice sequences were aligned with those of Arabidopsis, yeast and humans. ThiF domain, enzyme active site with the conserved Cysteine (shaded in red) residue and ubiquitin fold domain are documented. 


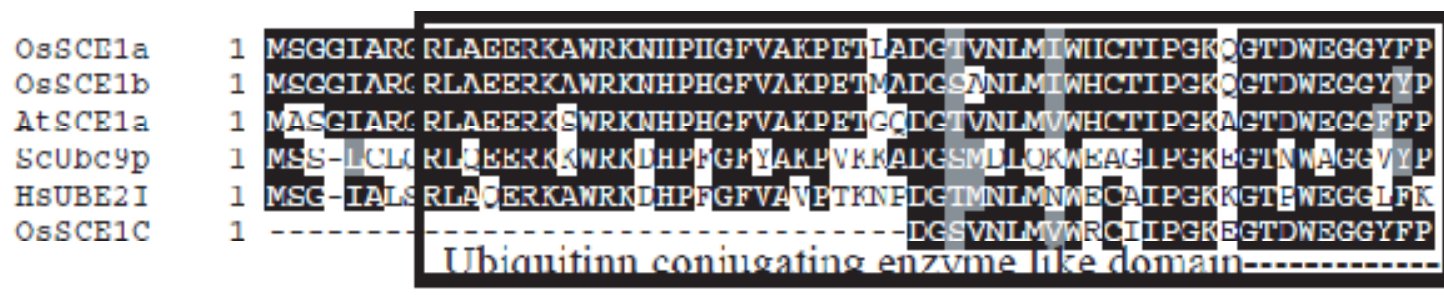

OSSCE1a 61 ITHFSEDYPSKPPKCKF POGF FHPNVYPSGTVCLSILNED GWRPAITVKOILVGI

OsSCE1b 61 ITLHFSEDYPSKPPKCKFPQGFFHPNVYPSGTVCL SILNED GWRPAITVKQILVGI

At SCE1a 61 ITMIIISGDYPSKPPKCKFPQGFPIPNVYPSGTVCL SILNIEDYGWRPAI TVKQILVGI

ScUbc9p

60 ITVEYPNEYPSKPPKVKFP 1 GF HPNVYP SGTICLSILNEDQDWRPAITLKR IV_GV

HsUBE2 I 60 IRMLEKDDYPS SPPKCKFE P PLFHPNVYPSGTVCLSII BEDKDWRPAI IIKQIL GI

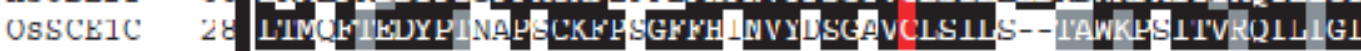

-

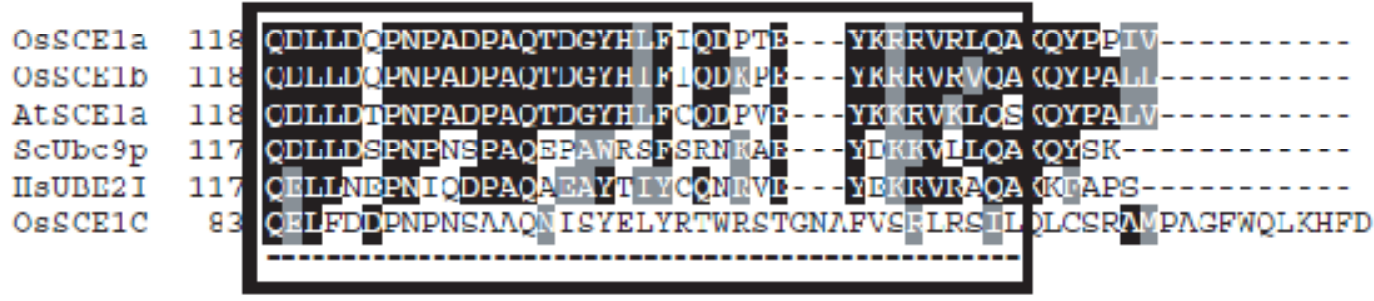

Figure 3-4. Rice SUMO E2 enzyme sequences were aligned with those of Arabidopsis, yeast and humans. Ubiquitin conjugating enzyme-like domains with active sites consisting of conserved cysteine residues (shaded in red) are depicted. 


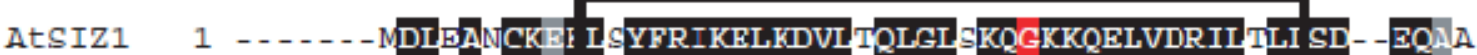

OSSIZ1 1 - - - -MPDLVSSCKDI I YYFRIKELKDILNQLGLPKQGKKODLIDRVLALI ITD- EQGQ

OsSIZ2 1 MALDPADDPLLADCKYI LNHFRIKELKDVLHQLGLPKOGRKQELVDKIIAVI SDQDEQDS

SAP domain-----------

AtSIZ1 52 RLL--SKKNIVAKEAVAKLVDDTYRKMQVSGASDLASKG GSSDTSNLKVKGEPEDP

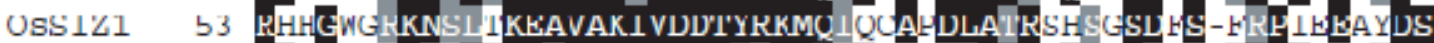

OSSIZ2 61 RIMGLPNKKMV VETVAKIVDDTFPKMISSTNAVPASRIQDDSGHI-VKPKRKSDDS

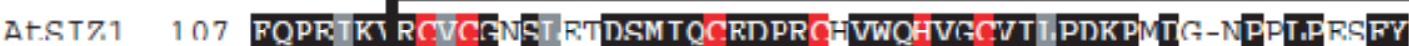

OSSIZ1 109 FOPEAKT RCICSSINVNDSMIOCEDCRCOVWOHINNCVLIPDKPGE- -SAEVPPVFY

OSSIZ2 117 AQLDVKI ROPCGYSNANDSMIKCEGECN TQQHVGCVI ISEKPAISVP PELPPHFY

PHD-Finger-.............................................

AtSIZ1 162 CEICRI TRADPFWVTVAPLSPVRIAITIPNDGASTMQSVERTFQITRADKDLIA

OSSIZ1 163 CELCRI STRADPFWVTAENPLLPVIFVS SGVTNDGTSVPQSVEKS FQLSRSDRETVO

OSSIZ2 173 CDNCE] TRADPFWVTVNHPVLPVSIIPCKVASDGEYAVQYFEKTE PLSRANGEMLQ

AtSIZ1 218 FEEYDVQAWCMLLNDKVIFRMOWPQYADLQVNGVPRA INRPGGQLLGVNGRDDGP

OSSIZ1 219 ROEYDLOVWCMLLNDKVOFRMOWPOYAEL HVNGISVRVVTRPGSOLLGINGRDDGP

OsSIZ2 229 KLEYDLQVWCILFND SVPFRMQWPLHSDIQINGIP IRVVNRQPICQLGVNGRDDGP

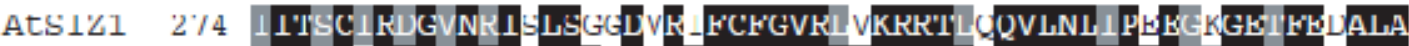

OSSIZ1 275 IITICSREINKIOLSRVDARTFCFGVRIAKRRTVAQVLNLVPKEAEGES FEHALA

OSSIZ2 205 VITAYVREG NNKIVLSR SD SRTFCL GVRIAKRRS VRQVLISLVPKLEDGENIF NALA

AtSIZ1 330 RVRRCIGGGGGDNADSDSDIEVVACFFC VNLRCPMSGSRIK VAGRELPCVHMGCF

OsSIZ1 331 RVRRCLGGDTAFNADSDSDLEVVABSV VNLRCPNSGSRVIIAGRFKPCIHMGCA

OsSIZ2 341 RVRRCVGGGTEADNADSDSDIEVVADSV VNLRCPMTGSRIKIAGRFKPCVHMGCE

MIZ/SP_Ring Zn finger-.....

AtSIZ1 386 DLEVFVLNQRSRKWQCPICLKN ISVEHVIVDPYFNRITSKMKHODEBVIEI EVKP

OSSIZ1 387 DLFTFVELNQRSRKWQCPICLKN ISLESLMIDPYFNRITSLLPN CNEDVNEVDVKP

OsSIZ2 397 DLEATVELNQRSRKWQCPICLKN ISLDNIIIDPYFNRITPLVQSGGDVSEIDVKP

(Continued) 


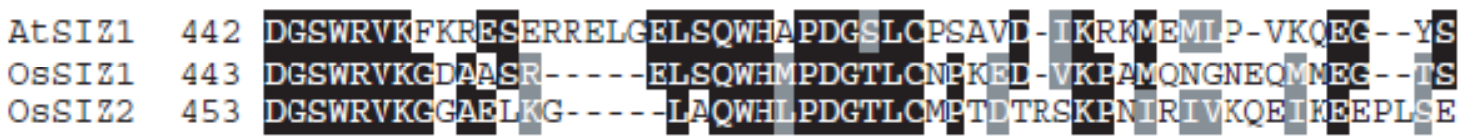

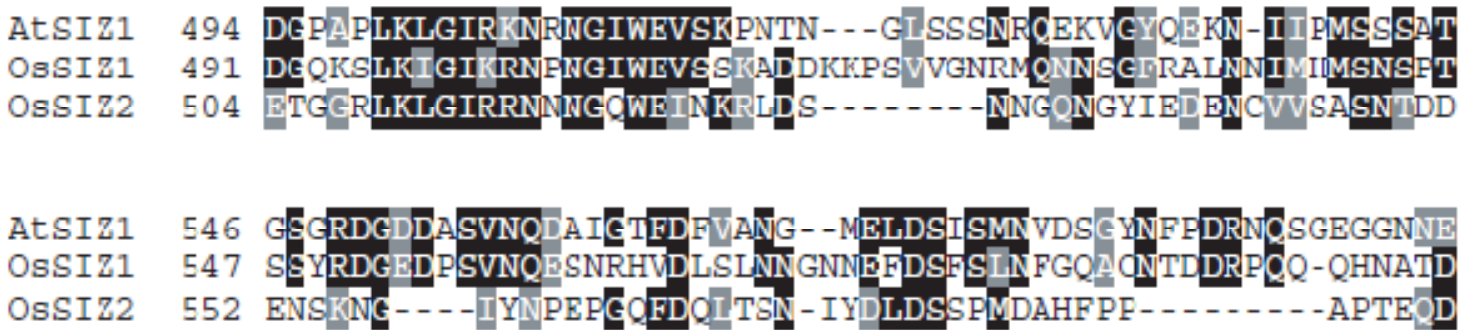

AtSIZ1 600 VIVLSDSDDENDLVITPEPAYSSGCOTDGETFPLNPPGIINSYNEDPHSIAG-GSS

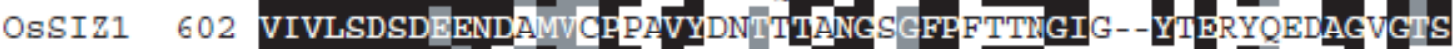
OsSIZ2 594 VIVLSDSDDDNVMVLSPGDVNFS SAHDNGNAFP PNPPEASGICGEOPE---GAGPD

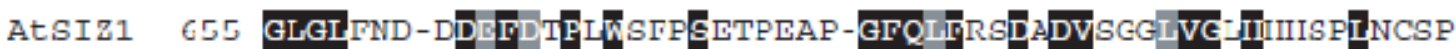
OsSIZ1 656 GLGLISNNVDDFEMNNWMHSSYDQPEOGFOFGGNDZDVHNTFVGSHNSFGLAPND OsSIZ2 647 VTSFLDG-FDDLELPFWESSSS--dDARETQVIDNQDEMDNFIVNHQFLH- - - - -

AtSIZ1 709 EINGGYTMAPETSNASVPVVPGTERSEANDGLVDPLAFGRDPSLQIFLPTKEOSSIZ1 712 YSLDCNVGVEEASVT--PDLSVCRNSNEMHGSLVDNPLALVGDDPSLQIFLPSCES OsSIZ2

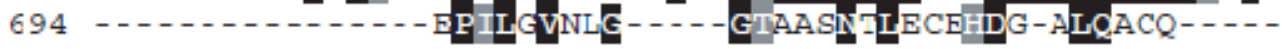

AtSIZ1 764 DASAOSEFKNQADMSNGLRSEDISLRLDSASGNHGDPATNG INSSHQMSTRE OSSIZ1 766 SVPLQEELSERANAPNGVOSDDISL IIAGGGGNE-EPA FAD-VNSQPQIPSTET OSSIZ2 723 - SSDQDEDQNQTCHDG--HSGDLTNLSIISII-- - -QDSITNGKNASQKRINCED

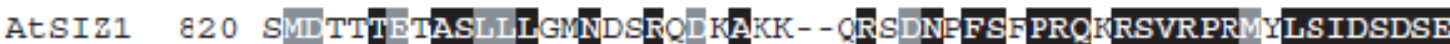
OsSIZ1 820 GIEPLTDAASAFLSTNIERRSGALNPFR I ENIFSH PRQPRSVRPROLSIDIDSE

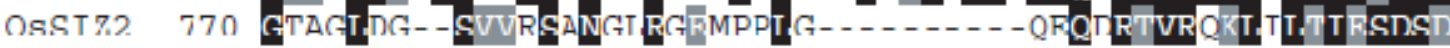

AtSIZ1 874 TMNRIIRQDTGV 885

OsSIZ1 876

OsSIZ2 \&14 -

Figure 3-5. PIAS type SUMO E3 enzyme sequences of rice aligned with those of Arabidopsis. The conserved SAP domain, PHD finger, and MIZ-SP Ring Zn finger domains are boxed, and the signature amino acid residues in each domain are shaded in red. 

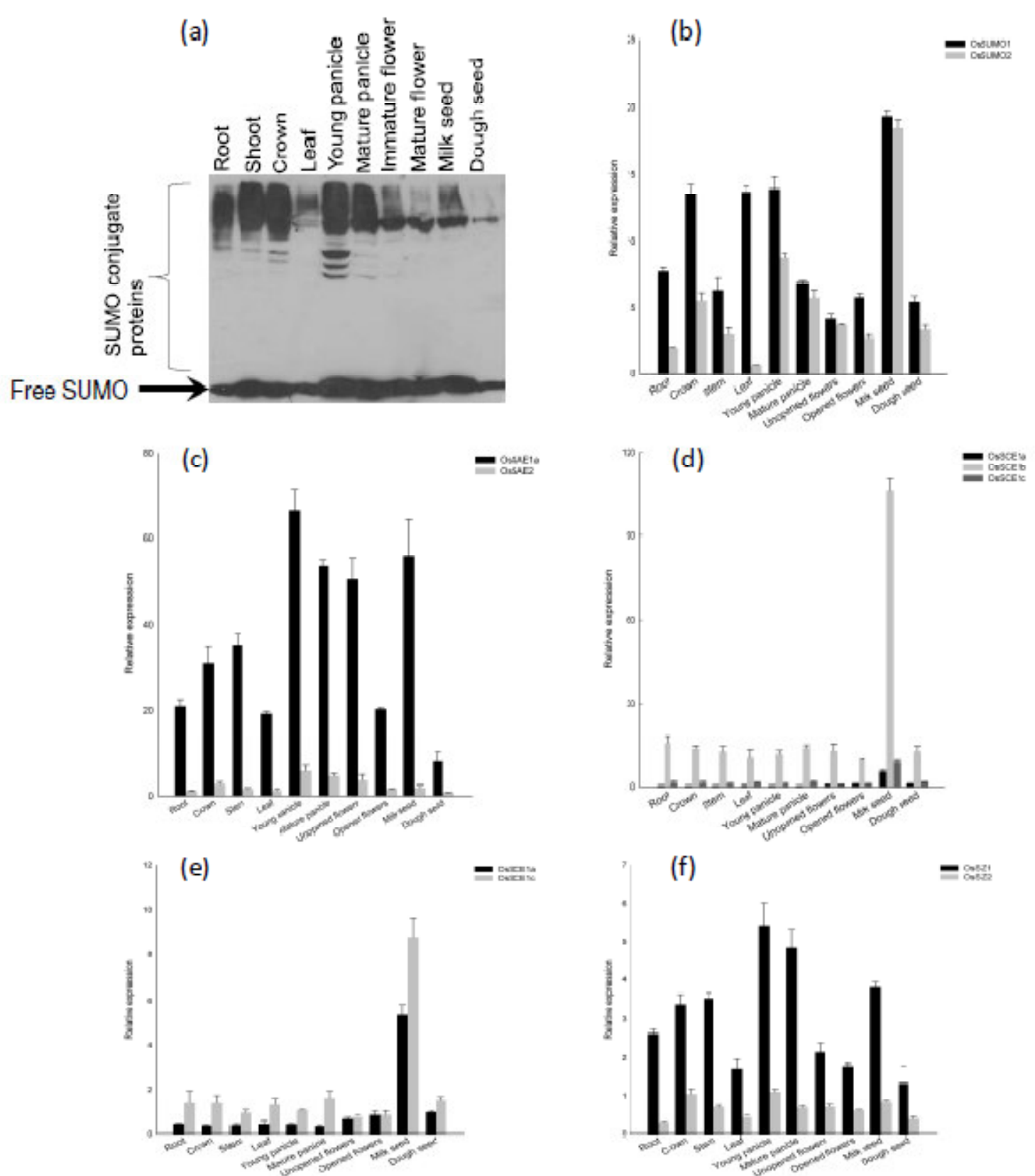

Figure 3-6. SUMOylation profiles and SUMO cascade component RNA accumulation in different tissues. (a) SUMOylation profiles in tissues from vegetative and reproductive tissues were determined by western blot analysis of $25 \mu \mathrm{g}$ of total proteins. Relative mRNA accumulation levels of rice (b) $S U M O$ genes (c) $E 1$ genes (d-i and d-ii) $E 2$ genes and (e) E3 genes were determined by quantitative real-time PCR by obtaining absolute 
copy number for each gene and normalizing with the absolute copy number of the $18 s r R N A$ gene. Note the differences in the scale of the Y-axis in the different graphs. Note that all of the SUMO components are expressed in all tissues and the majority of them are highly abundant in reproductive tissues of young panicles and milk seeds. 


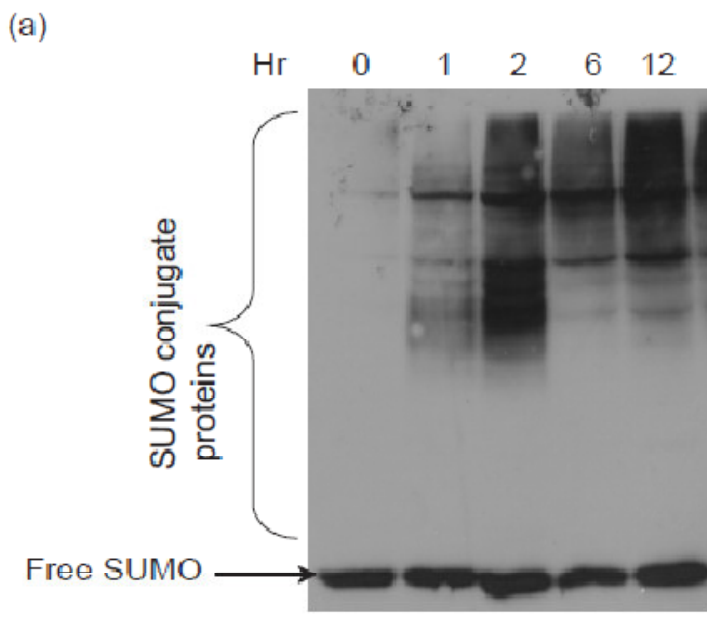

(b)

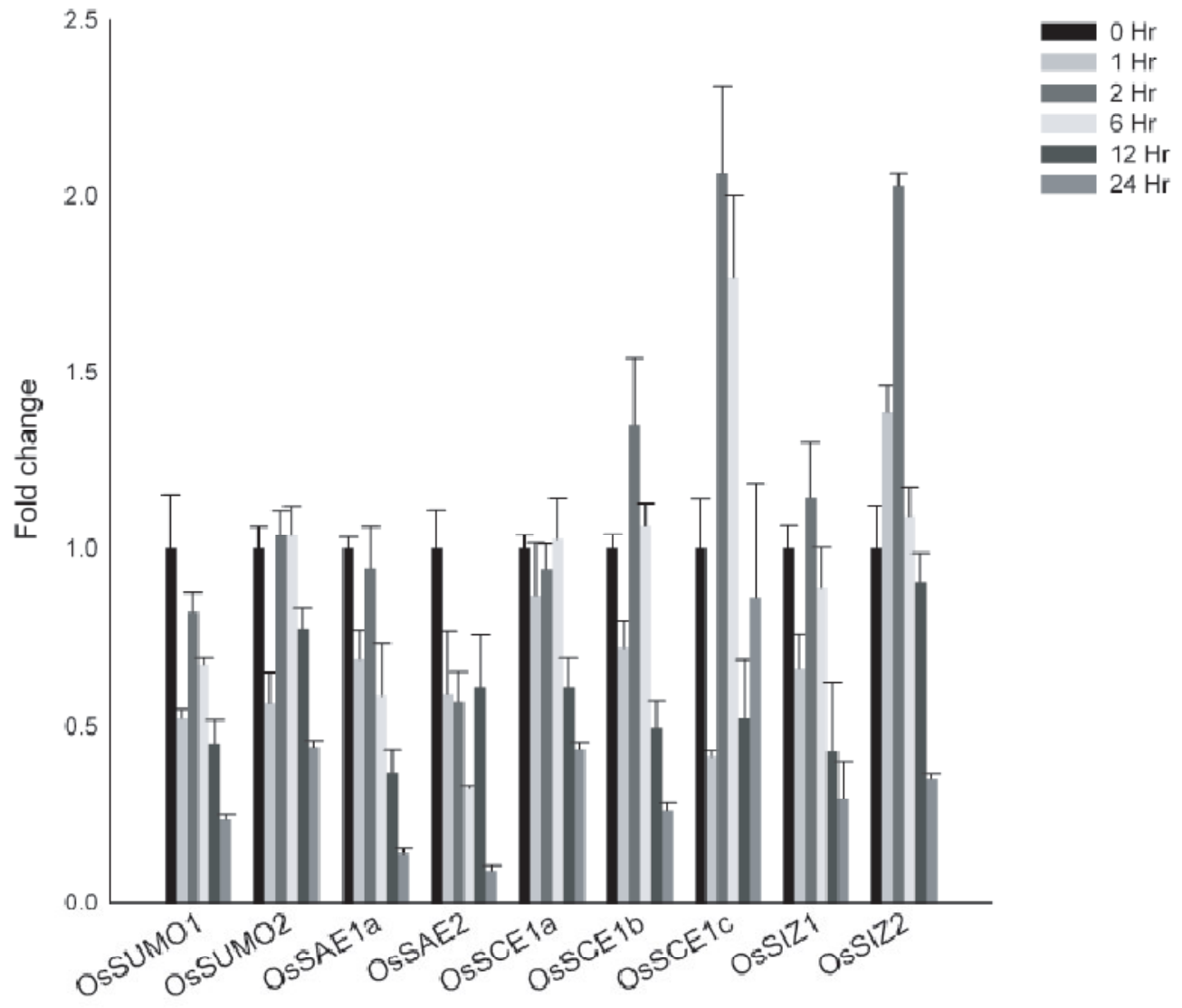

Figure 3-7. Cold stress induces accumulation of SUMO conjugates and affects SUMO component gene expression. (a) In planta SUMOylation profiles in root tissue of 1 week old seedlings grown in a growth chamber maintained at $30^{\circ} \mathrm{C}(0 \mathrm{hr}$ time point $)$ and then transferred into cold water maintained in a growth chamber at $4^{\circ} \mathrm{C}$. Fifteen $\mu \mathrm{g}$ of total proteins were isolated and subjected to western blot analysis. Note the accumulation of 
high molecular weight SUMO conjugates after cold stress treatment. (b) Changes in mRNA levels of rice SUMOylation components with cold treatment were determined by quantitative real-time PCR. Changes in transcript abundance were represented as fold change by calibrating the relative mRNA levels of each time point with the relative mRNA level of the $0 \mathrm{hr}$ time point. Note the down regulation of transcript levels for SUMO and E1 genes. Among E2 and E3 genes, OsSCE1b, OsSCE1c and OsSIZ2 are transiently up regulated. 
(a)

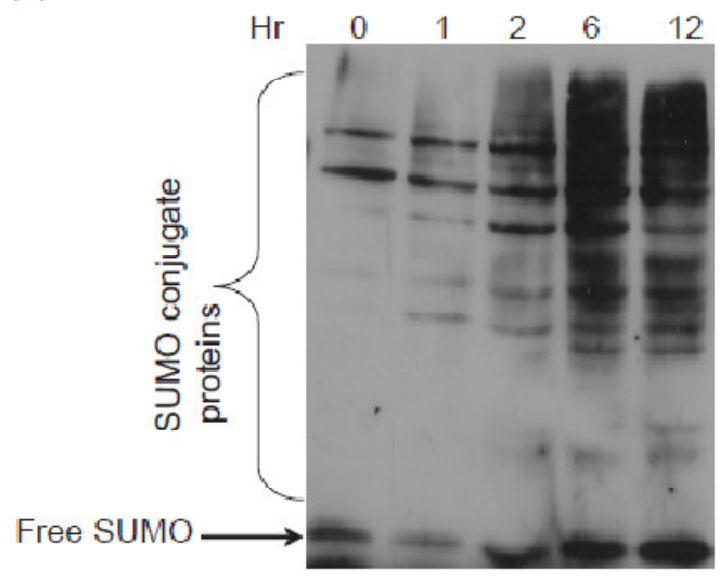

(b)

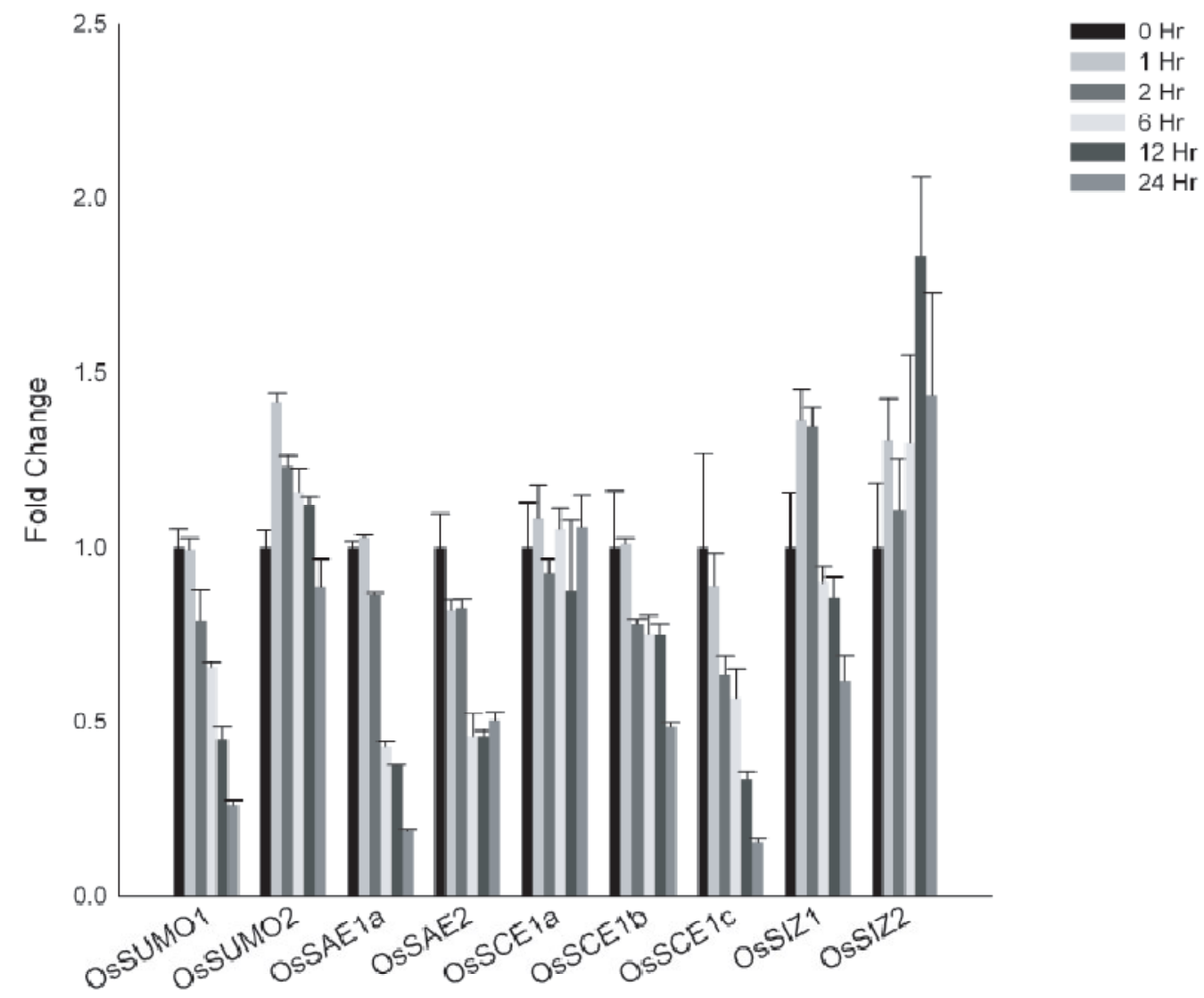

Figure 3-8. Salt stress induces accumulation of SUMO conjugates and effects SUMO component gene expression. (a) Western blot analysis of $15 \mu \mathrm{g}$ of total proteins from 1 week old seedling root tissue grown in tap water $(0 \mathrm{hr})$ and after transferring to $250 \mathrm{mM}$ salt solution. Note the accumulation of high molecular weight SUMO conjugates after transferring to salt solution. (b) Changes in mRNA levels of rice SUMOylation 
components with salt stress as determined by quantitative real-time PCR. Fold change was calculated as described for Fig 2B. Note the down regulation of SUMO and E1 and E2 (except OsSCE1a) encoding genes. Among the E3 genes, OsSIZ1 and OsSIZ2 were up-regulated. 
(a)

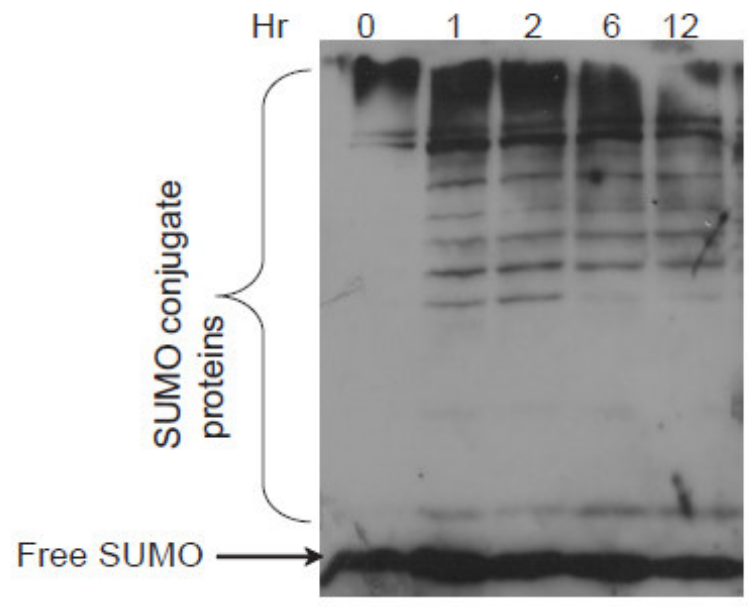

(b)

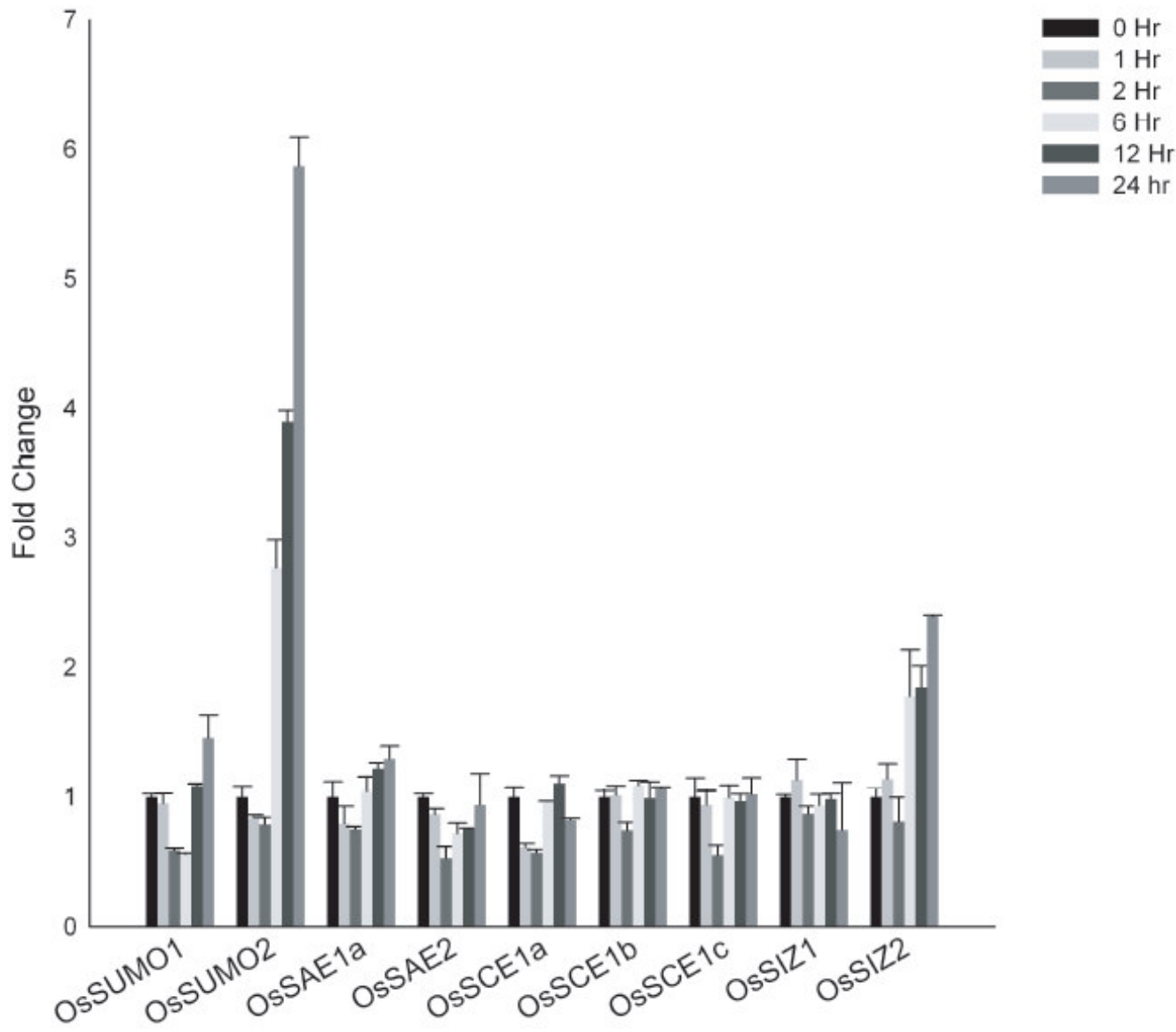

Figure 3-9. ABA induces accumulation of SUMO conjugates and affects SUMO component gene expression. (a) SUMO conjugate levels from 1 week old seedling root tissue grown in tap water $(0 \mathrm{hr})$ and after transferring to $50 \mathrm{mM}$ ABA solution were 
determined by western blotting with $15 \mu \mathrm{g}$ of total protein extracts. Note the accumulation of high molecular weight SUMO conjugates after transferring to ABA solution (b) Changes in mRNA levels of rice SUMOylation components were determined by quantitative real-time PCR analysis. Fold change was calculated as described for Fig 2b. OsSUMO2 and OSSIZ2 exhibited five and two fold up-regulation in response to ABA. 


\section{CONCLUSIONS}

The aim of the first part of the study was to understand the role of rice cold shock domain proteins (OsCSPs) in plant abiotic stress responses and during plant development. The conclusions that can be drawn from this study are:

The rice genome contains two cold shock domain proteins

Rice CSPs like CSPs from other organisms exhibit a ssDNA binding

Similar to the bacterial and winter wheat CSPs, rice CSPs are capable of functioning as RNA chaperones

D Unlike winter wheat and Arabidopsis CSP transcripts, OSCSP transcripts are only transiently and marginally increased in response to cold stress

$>$ OsCSPs do not accumulate in response to cold stress. This is in contrast to the results obtained for CSPs from winter wheat and Arabidopsis

> Comparison of the expression data of CSPs from rice with that from cold acclimation capable plants like Arabidopsis and winter wheat suggests that CSPs might be involved with the ability for cold acclimation in higher plants

D During development, rice CSPs and their transcripts are highly expressed in the reproductive tissues implicating a role in plant reproductive development

$>$ Rice CSPs are also highly expressed in actively growing tissues which commonly exhibit meristematic activity, thus implicating a role for these proteins in plant growth and development

The aim of the second part of this study was to determine if plant CSPs are modified by SUMOylation and to decipher the effects of this modification. Conclusions from this study are:

Both rice CSPs contain SUMOylation motifs and are capable of undergoing SUMOylation

Among the four Arabidopsis CSPs, only AtCSP1(At4g36020) contains consensus SUMOylation motifs

AtCSP1 protein is SUMOylated on Lysine residue 83

Modification by SUMOylation appears to affect the sub-cellular localization of AtCSP1 
In the third part of this study, I aimed to characterize the sequence and transcriptional regulation of individual components of the SUMO conjugation system of rice. I also characterized the SUMO conjugate profiles during plant development and stress conditions. The conclusions from this study are:

Rice contains all the components required for SUMO conjugation: SUMO proteins, E1 activation enzymes, E2 conjugation enzymes, and E3 SUMO ligation enzymes

> Sequence analysis revealed that rice SUMO proteins, E1 and E2 enzymes show similarity to those of Arabidopsis, yeast and human sequences and contain all conserved motifs.

PIAS type E3 enzymes from rice show high similarity to those of Arabidopsis. Rice and Arabidopsis PIAS-type E3 ligases contain extra domains that are not present in yeast and animal PIAS-type E3 ligases

$>$ During development, the highest levels of SUMO conjugates are found in panicle tissues, suggesting an important role in plant reproductive development

All of the SUMO cascade components are ubiquitously expressed indicating the essentiality of the SUMOylation system in all plant tissues

$>$ The majority of the SUMOylation cascade components are preferentially expressed in reproductive tissues like developing seeds and panicles which also implicate important roles for the SUMOylation system during the reproductive development of rice

The SUMOylation system of rice responds to abiotic stress conditions like cold, salt and $\mathrm{ABA}$ treatments by accumulating large amounts of SUMO conjugate proteins. This indicates the importance of SUMO conjugated proteins in mediating stress responses in rice

In response to stress conditions, the majority of the SUMOylation components are transcriptionally down-regulated. However, the SUMO ligase gene (OsSIZ2) is transiently up-regulated during all of these stress treatments. Considering the importance of SUMO ligases in increasing the efficiency and specificity of SUMO conjugation, OsSIZ2 may play an important role in the accumulation of SUMO conjugate proteins during stress conditions. 


\section{LITERATURE CITED}

Anckar, J., Hietakangas, V., Denessiouk, K., Thiele, D.J., Johnson, M.S., and Sistonen, L. (2006). Inhibition of DNA binding by differential sumoylation of heat shock factors. Mol Cell Biol 26, 955-964.

Andrews, E.A., Palecek, J., Sergeant, J., Taylor, E., Lehmann, A.R., and Watts, F.Z. (2005). Nse2, a component of the Smc5-6 complex, is a SUMO ligase required for the response to DNA damage. Mol Cell Biol 25, 185-196.

Aravind, L., and Koonin, E.V. (2000). SAP - a putative DNA-binding motif involved in chromosomal organization. Trends Biochem Sci 25, 112-114.

Baba, D., Maita, N., Jee, J.G., Uchimura, Y., Saitoh, H., Sugasawa, K., Hanaoka, F., Tochio, H., Hiroaki, H., and Shirakawa, M. (2005). Crystal structure of thymine DNA glycosylase conjugated to SUMO-1. Nature 435, 979-982.

Bachant, J., Alcasabas, A., Blat, Y., Kleckner, N., and Elledge, S.J. (2002). The SUMO-1 isopeptidase Smt4 is linked to centromeric cohesion through SUMO-1 modification of DNA topoisomerase II. Mol Cell 9, 1169-1182.

Bader, A.G., and Vogt, P.K. (2005). Inhibition of protein synthesis by Y box-binding protein 1 blocks oncogenic cell transformation. Mol Cell Biol 25, 2095-2106.

Bae, W., Phadtare, S., Severinov, K., and Inouye, M. (1999). Characterization of Escherichia coli cspE, whose product negatively regulates transcription of cspA, the gene for the major cold shock protein. Mol Microbiol 31, 1429-1441.

Bae, W., Xia, B., Inouye, M., and Severinov, K. (2000). Escherichia coli CspA-family RNA chaperones are transcription antiterminators. Proc Natl Acad Sci USA 97, 7784-7789.

Baek, S.H. (2006). A novel link between SUMO modification and cancer metastasis. Cell Cycle 5, 1492-1495.

Bayer, P., Arndt, A., Metzger, S., Mahajan, R., Melchior, F., Jaenicke, R., and Becker, J. (1998). Structure determination of the small ubiquitin-related modifier SUMO-1. J Mol Biol 280, 275-286.

Bernier-Villamor, V., Sampson, D.A., Matunis, M.J., and Lima, C.D. (2002). Structural basis for E2-mediated SUMO conjugation revealed by a complex between ubiquitin-conjugating enzyme Ubc9 and RanGAP1. Cell 108, 345-356.

Best, J.L., Ganiatsas, S., Agarwal, S., Changou, A., Salomoni, P., Shirihai, O., Meluh, P.B., Pandolfi, P.P., and Zon, L.I. (2002). SUMO-1 protease-1 regulates gene transcription through PML. Mol Cell 10, 843-855.

Bienz, M. (2006). The PHD finger, a nuclear protein-interaction domain. Trends Biochem Sci 31, 35-40.

Blobel, G. (1973). A protein of molecular weight 78,000 bound to the polyadenylate region of eukaryotic messenger RNAs. Proc Natl Acad Sci USA 70, 924-928.

Bloch, D.B., Chiche, J.D., Orth, D., de la Monte, S.M., Rosenzweig, A., and Bloch, K.D. (1999). Structural and functional heterogeneity of nuclear bodies. Mol Cell Biol 19, 4423-4430.

Boggio, R., Colombo, R., Hay, R.T., Draetta, G.F., and Chiocca, S. (2004). A mechanism for inhibiting the SUMO pathway. Mol Cell 16, 549-561.

Boggio, R., and Chiocca, S. (2005). Gam1 and the SUMO pathway. Cell Cycle 4, 533535. 
Boggio, R., Passafaro, A., and Chiocca, S. (2007). Targeting SUMO E1 to ubiquitin ligases: a viral strategy to counteract sumoylation. J Biol Chem 282, 1537615382.

Bono, E., Compagno, V., Proia, P., Raimondi, L., Schiera, G., Favaloro, V., Campo, V., Donatelli, M., and Di Liegro, I. (2007). Thyroid hormones induce sumoylation of the cold shock domain-containing protein PIPPin in developing rat brain and in cultured neurons. Endocrinology 148, 252-257.

Bossis, G., Malnou, C.E., Farras, R., Andermarcher, E., Hipskind, R., Rodriguez, M., Schmidt, D., Muller, S., Jariel-Encontre, I., and Piechaczyk, M. (2005). Down-regulation of c-Fos/c-Jun AP-1 dimer activity by sumoylation. Mol Cell Biol 25, 6964-6979.

Bossis, G., and Melchior, F. (2006). SUMO: regulating the regulator. Cell Div 1, 13.

Bossis, G., and Melchior, F. (2006). Regulation of SUMOylation by reversible oxidation of SUMO conjugating enzymes. Mol Cell 21, 349-357.

Bouras, T., Fu, M., Sauve, A.A., Wang, F., Quong, A.A., Perkins, N.D., Hay, R.T., Gu, W., and Pestell, R.G. (2005). SIRT1 deacetylation and repression of p300 involves lysine residues 1020/1024 within the cell cycle regulatory domain 1 . J Biol Chem 280, 10264-10276.

Brandi, A., Pietroni, P., Gualerzi, C.O., and Pon, C.L. (1996). Post-transcriptional regulation of CspA expression in Escherichia coli. Mol Microbiol 19, 231-240.

Budhiraja, R., Hermkes, R., Muller, S., Schmidt, J., Colby, T., Panigrahi, K., Coupland, G., and Bachmair, A. (2009). Substrates Related to Chromatin and to RNA-Dependent Processes Are Modified by Arabidopsis SUMO Isoforms That Differ in a Conserved Residue with Influence on Desumoylation. Plant Physiol 149, 1529-1540.

Carbia-Nagashima, A., and Arzt, E. (2004). Intracellular proteins and mechanisms involved in the control of gp130/JAK/STAT cytokine signaling. IUBMB Life 56, 83-88.

Castillo, A.G., Kong, L.J., Hanley-Bowdoin, L., and Bejarano, E.R. (2004). Interaction between a geminivirus replication protein and the plant sumoylation system. J Virol 78, 2758-2769.

Catala, R., Ouyang, J., Abreu, I.A., Hu, Y., Seo, H., Zhang, X., and Chua, N.H. (2007). The Arabidopsis E3 SUMO ligase SIZ1 regulates plant growth and drought responses. Plant Cell 19, 2952-2966.

Chaikam, V., and Karlson, D. (2008). Functional characterization of two cold shock domain proteins from Oryza sativa. Plant Cell Environ 31, 995-1006.

Chansky, H.A., Hu, M., Hickstein, D.D., and Yang, L. (2001). Oncogenic TLS/ERG and EWS/Fli-1 fusion proteins inhibit RNA splicing mediated by YB-1 protein. Cancer Res 61, 3586-3590.

Chen, C.Y., Gherzi, R., Andersen, J.S., Gaietta, G., Jurchott, K., Royer, H.D., Mann, M., and Karin, M. (2000). Nucleolin and YB-1 are required for JNKmediated interleukin-2 mRNA stabilization during T-cell activation. Genes Dev 14, 1236-1248.

Chen, A., Wang, P.Y., Yang, Y.C., Huang, Y.H., Yeh, J.J., Chou, Y.H., Cheng, J.T., Hong, Y.R., and Li, S.S. (2006). SUMO regulates the cytoplasmonuclear transport of its target protein Daxx. J Cell Biochem 98, 895-911. 
Chinnusamy, V., Zhu, J., and Zhu, J.K. (2006). Gene regulation during cold acclimation in plants. Physiologia Plantarum 126, 52-61.

Chiu, W., Niwa, Y., Zeng, W., Hirano, T., Kobayashi, H., and Sheen, J. (1996). Engineered GFP as a vital reporter in plants. Curr Biol 6, 325-330.

Chosed, R., Mukherjee, S., Lois, L.M., and Orth, K. (2006). Evolution of a signalling system that incorporates both redundancy and diversity: Arabidopsis SUMOylation. Biochem J 398, 521-529.

Chung, T.L., Hsiao, H.H., Yeh, Y.Y., Shia, H.L., Chen, Y.L., Liang, P.H., Wang, A.H., Khoo, K.H., and Shoei-Lung Li, S. (2004). In vitro modification of human centromere protein CENP-C fragments by small ubiquitin-like modifier (SUMO) protein: definitive identification of the modification sites by tandem mass spectrometry analysis of the isopeptides. J Biol Chem 279, 39653-39662.

Colby, T., Matthai, A., Boeckelmann, A., and Stuible, H.P. (2006). SUMOconjugating and SUMO-deconjugating enzymes from Arabidopsis. Plant Physiol 142, 318-332.

Coles, L.S., Lambrusco, L., Burrows, J., Hunter, J., Diamond, P., Bert, A.G., Vadas, M.A., and Goodall, G.J. (2005). Phosphorylation of cold shock domain/Y-box proteins by ERK2 and GSK3beta and repression of the human VEGF promoter. FEBS Lett 579, 5372-5378.

Comerford, K.M., Leonard, M.O., Karhausen, J., Carey, R., Colgan, S.P., and Taylor, C.T. (2003). Small ubiquitin-related modifier-1 modification mediates resolution of CREB-dependent responses to hypoxia. Proc Natl Acad Sci USA 100, 986-991.

Conti, L., Price, G., O'Donnell, E., Schwessinger, B., Dominy, P., and Sadanandom, A. (2008). Small ubiquitin-like modifier proteases OVERLY TOLERANT TO SALT1 and -2 regulate salt stress responses in Arabidopsis. Plant Cell 20, 28942908.

Dannull, J., Surovoy, A., Jung, G., and Moelling, K. (1994). Specific binding of HIV-1 nucleocapsid protein to PSI RNA in vitro requires N-terminal zinc finger and flanking basic amino acid residues. EMBO J 13, 1525-1533.

Davies, H.G., Giorgini, F., Fajardo, M.A., and Braun, R.E. (2000). A sequencespecific RNA binding complex expressed in murine germ cells contains MSY2 and MSY4. Dev Biol 221, 87-100.

Desterro, J.M., Rodriguez, M.S., and Hay, R.T. (1998). SUMO-1 modification of IkappaBalpha inhibits NF-kappaB activation. Mol Cell 2, 233-239.

Desterro, J.M., Rodriguez, M.S., Kemp, G.D., and Hay, R.T. (1999). Identification of the enzyme required for activation of the small ubiquitin-like protein SUMO-1. J Biol Chem 274, 10618-10624.

Deyrieux, A.F., Rosas-Acosta, G., Ozbun, M.A., and Wilson, V.G. (2007). Sumoylation dynamics during keratinocyte differentiation. J Cell Sci 120, 125136.

Didier, D.K., Schiffenbauer, J., Woulfe, S.L., Zacheis, M., and Schwartz, B.D. (1988). Characterization of the cDNA encoding a protein binding to the major histocompatibility complex class II Y box. Proc Natl Acad Sci USA 85, 7322 7326.

Dohmen, R.J., Stappen, R., McGrath, J.P., Forrova, H., Kolarov, J., Goffeau, A., 
and Varshavsky, A. (1995). An essential yeast gene encoding a homolog of ubiquitin-activating enzyme. J Biol Chem 270, 18099-18109.

Dooki, A.D., Mayer-Posner, F.J., Askari, H., Zaiee, A.A., and Salekdeh, G.H. (2006). Proteomic responses of rice young panicles to salinity. Proteomics 6, 6498-6507.

Downes, B., and Vierstra, R.D. (2005). Post-translational regulation in plants employing a diverse set of polypeptide tags. Biochem Soc Trans 33, 393-399.

Dreyfuss, G. (1986). Structure and function of nuclear and cytoplasmic ribonucleoprotein particles. Annu Rev Cell Biol 2, 459-498.

Du, J.X., Bialkowska, A.B., McConnell, B.B., and Yang, V.W. (2008). SUMOylation regulates nuclear localization of Kruppel-like factor 5. J Biol Chem 283, 3199132002.

En-Nia, A., Yilmaz, E., Klinge, U., Lovett, D.H., Stefanidis, I., and Mertens, P.R. (2005). Transcription factor YB-1 mediates DNA polymerase alpha gene expression. J Biol Chem 280, 7702-7711.

Espinosa, J.M., Portal, D., Lobo, G.S., Pereira, C.A., Alonso, G.D., Gomez, E.B., Lan, G.H., Pomar, R.V., Flawia, M.M., and Torres, H.N. (2003). Trypanosoma cruzi poly-zinc finger protein: a novel DNA/RNA-binding CCHCzinc finger protein. Mol Biochem Parasitol 131, 35-44.

Evdokimova, V.M., Wei, C.L., Sitikov, A.S., Simonenko, P.N., Lazarev, O.A., Vasilenko, K.S., Ustinov, V.A., Hershey, J.W., and Ovchinnikov, L.P. (1995). The major protein of messenger ribonucleoprotein particles in somatic cells is a member of the Y-box binding transcription factor family. J Biol Chem 270, 31863192.

Evdokimova, V.M., Kovrigina, E.A., Nashchekin, D.V., Davydova, E.K., Hershey, J.W., and Ovchinnikov, L.P. (1998). The major core protein of messenger ribonucleoprotein particles (p50) promotes initiation of protein biosynthesis in vitro. J Biol Chem 273, 3574-3581.

Evdokimova, V.M., and Ovchinnikov, L.P. (1999). Translational regulation by Y-box transcription factor: involvement of the major mRNA-associated protein, p50. Int J Biochem Cell Biol 31, 139-149.

Evdokimova, V., Ruzanov, P., Imataka, H., Raught, B., Svitkin, Y., Ovchinnikov, L.P., and Sonenberg, N. (2001). The major mRNA-associated protein YB-1 is a potent 5' cap-dependent mRNA stabilizer. EMBO J 20, 5491-5502.

Evdokimova, V., Ruzanov, P., Anglesio, M.S., Sorokin, A.V., Ovchinnikov, L.P., Buckley, J., Triche, T.J., Sonenberg, N., and Sorensen, P.H. (2006). Aktmediated YB-1 phosphorylation activates translation of silent mRNA species. Mol Cell Biol 26, 277-292.

Fang, L., Jiang, W., Bae, W., and Inouye, M. (1997). Promoter-independent coldshock induction of cspA and its derepression at 37 degrees $\mathrm{C}$ by mRNA stabilization. Mol Microbiol 23, 355-364.

Feng, W., Tejero, R., Zimmerman, D.E., Inouye, M., and Montelione, G.T. (1998). Solution NMR structure and backbone dynamics of the major cold-shock protein (CspA) from Escherichia coli: evidence for conformational dynamics in the single-stranded RNA-binding site. Biochemistry 37, 10881-10896.

Feng, Y., Huang, H., Liao, J., and Cohen, S.N. (2001). Escherichia coli poly(A)binding proteins that interact with components of degradosomes or impede RNA 
decay mediated by polynucleotide phosphorylase and RNase E. J Biol Chem 276, 31651-31656.

Finn, R.D., Tate, J., Mistry, J., Coggill, P.C., Sammut, S.J., Hotz, H.R., Ceric, G., Forslund, K., Eddy, S.R., Sonnhammer, E.L., and Bateman, A. (2008). The Pfam protein families database. Nucleic Acids Res 36, D281-288.

Fu, C., Ahmed, K., Ding, H., Ding, X., Lan, J., Yang, Z., Miao, Y., Zhu, Y., Shi, Y., Zhu, J., Huang, H., and Yao, X. (2005). Stabilization of PML nuclear localization by conjugation and oligomerization of SUMO-3. Oncogene 24, 54015413.

Fusaro, A.F., Bocca, S.N., Ramos, R.L., Barroco, R.M., Magioli, C., Jorge, V.C., Coutinho, T.C., Rangel-Lima, C.M., De Rycke, R., Inze, D., Engler, G., and Sachetto-Martins, G. (2007). AtGRP2, a cold-induced nucleo-cytoplasmic RNA-binding protein, has a role in flower and seed development. Planta 225, 1339-1351.

Garcia-Dominguez, M., March-Diaz, R., and Reyes, J.C. (2008). The PHD domain of plant PIAS proteins mediates sumoylation of bromodomain GTE proteins. J Biol Chem 283, 21469-21477.

Gaudreault, I., Guay, D., and Lebel, M. (2004). YB-1 promotes strand separation in vitro of duplex DNA containing either mispaired bases or cisplatin modifications, exhibits endonucleolytic activities and binds several DNA repair proteins. Nucleic Acids Res 32, 316-327.

Geiss-Friedlander, R., and Melchior, F. (2007). Concepts in sumoylation: a decade on. Nat Rev Mol Cell Biol 8, 947-956.

Gill, G. (2003). Post-translational modification by the small ubiquitin-related modifier SUMO has big effects on transcription factor activity. Curr Opin Genet Dev 13, 108-113.

Gill, G. (2004). SUMO and ubiquitin in the nucleus: different functions, similar mechanisms? Genes Dev 18, 2046-2059.

Gill, G. (2005). Something about SUMO inhibits transcription. Curr Opin Genet Dev 15, 536-541.

Girdwood, D., Bumpass, D., Vaughan, O.A., Thain, A., Anderson, L.A., Snowden, A.W., Garcia-Wilson, E., Perkins, N.D., and Hay, R.T. (2003). P300 transcriptional repression is mediated by SUMO modification. Mol Cell 11, 10431054.

Giuliodori, A.M., Gualerzi, C.O., Soto, S., Vila, J., and Tavio, M.M. (2007). Review on bacterial stress topics. Ann N Y Acad Sci 1113, 95-104.

Goldenberg, D., Azar, I., and Oppenheim, A.B. (1996). Differential mRNA stability of the cspA gene in the cold-shock response of Escherichia coli. Mol Microbiol 19, 241-248.

Goldstein, J., Pollitt, N.S., and Inouye, M. (1990). Major cold shock protein of Escherichia coli. Proc Natl Acad Sci USA 87, 283-287.

Gong, L., Li, B., Millas, S., and Yeh, E.T. (1999). Molecular cloning and characterization of human AOS1 and UBA2, components of the sentrin-activating enzyme complex. FEBS Lett 448, 185-189.

Goodson, M.L., Hong, Y., Rogers, R., Matunis, M.J., Park-Sarge, O.K., and Sarge, K.D. (2001). Sumo-1 modification regulates the DNA binding activity of heat 
shock transcription factor 2, a promyelocytic leukemia nuclear body associated transcription factor. J Biol Chem 276, 18513-18518.

Gorelick, R.J., Henderson, L.E., Hanser, J.P., and Rein, A. (1988). Point mutants of Moloney murine leukemia virus that fail to package viral RNA: evidence for specific RNA recognition by a "zinc finger-like" protein sequence. Proc Natl Acad Sci USA 85, 8420-8424.

Graumann, P., and Marahiel, M.A. (1996). A case of convergent evolution of nucleic acid binding modules. Bioessays 18, 309-315.

Graumann, P.L., and Marahiel, M.A. (1998). A superfamily of proteins that contain the cold-shock domain. Trends Biochem Sci 23, 286-290.

Gregoire, S., Tremblay, A.M., Xiao, L., Yang, Q., Ma, K., Nie, J., Mao, Z., Wu, Z., Giguere, V., and Yang, X.J. (2006). Control of MEF2 transcriptional activity by coordinated phosphorylation and sumoylation. J Biol Chem 281, 4423-4433.

Gu, W., Tekur, S., Reinbold, R., Eppig, J.J., Choi, Y.C., Zheng, J.Z., Murray, M.T., and Hecht, N.B. (1998). Mammalian male and female germ cells express a germ cell-specific Y-Box protein, MSY2. Biol Reprod 59, 1266-1274.

Guo, B., Yang, S.H., Witty, J., and Sharrocks, A.D. (2007). Signalling pathways and the regulation of SUMO modification. Biochem Soc Trans 35, 1414-1418.

Hahn, M., and Walbot, V. (1989). Effects of Cold-Treatment on Protein Synthesis and mRNA Levels in Rice Leaves. Plant Physiol 91, 930-938.

Hang, J., and Dasso, M. (2002). Association of the human SUMO-1 protease SENP2 with the nuclear pore. J Biol Chem 277, 19961-19966.

Hanna, M.M., and Liu, K. (1998). Nascent RNA in transcription complexes interacts with CspE, a small protein in E. coli implicated in chromatin condensation. J Mol Biol 282, 227-239.

Hardeland, U., Steinacher, R., Jiricny, J., and Schar, P. (2002). Modification of the human thymine-DNA glycosylase by ubiquitin-like proteins facilitates enzymatic turnover. Embo J 21, 1456-1464.

Harder, Z., Zunino, R., and McBride, H. (2004). Sumo1 conjugates mitochondrial substrates and participates in mitochondrial fission. Curr Biol 14, 340-345.

Hay, R.T. (2005). SUMO: a history of modification. Mol Cell 18, 1-12.

Hecker, C.M., Rabiller, M., Haglund, K., Bayer, P., and Dikic, I. (2006). Specification of SUMO1- and SUMO2-interacting motifs. J Biol Chem 281, $16117-16127$.

Heun, P. (2007). SUMOrganization of the nucleus. Curr Opin Cell Biol 19, 350-355.

Hietakangas, V., Ahlskog, J.K., Jakobsson, A.M., Hellesuo, M., Sahlberg, N.M., Holmberg, C.I., Mikhailov, A., Palvimo, J.J., Pirkkala, L., and Sistonen, L. (2003). Phosphorylation of serine 303 is a prerequisite for the stress-inducible SUMO modification of heat shock factor 1. Mol Cell Biol 23, 2953-2968.

Hietakangas, V., Anckar, J., Blomster, H.A., Fujimoto, M., Palvimo, J.J., Nakai, A., and Sistonen, L. (2006). PDSM, a motif for phosphorylation-dependent SUMO modification. Proc Natl Acad Sci USA 103, 45-50.

Hilgarth, R.S., Murphy, L.A., Skaggs, H.S., Wilkerson, D.C., Xing, H., and Sarge, K.D. (2004). Regulation and function of SUMO modification. J Biol Chem 279, 53899-53902.

Hochstrasser, M. (2001). SP-RING for SUMO: new functions bloom for a ubiquitin-like 
protein. Cell 107, 5-8.

Hoege, C., Pfander, B., Moldovan, G.L., Pyrowolakis, G., and Jentsch, S. (2002). RAD6-dependent DNA repair is linked to modification of PCNA by ubiquitin and SUMO. Nature 419, 135-141.

Horn, G., Hofweber, R., Kremer, W., and Kalbitzer, H.R. (2007). Structure and function of bacterial cold shock proteins. Cell Mol Life Sci 64, 1457-1470.

Hu, K.H., Liu, E., Dean, K., Gingras, M., DeGraff, W., and Trun, N.J. (1996). Overproduction of three genes leads to camphor resistance and chromosome condensation in Escherichia coli. Genetics 143, 1521-1532.

Huang, T.T., Wuerzberger-Davis, S.M., Wu, Z.H., and Miyamoto, S. (2003). Sequential modification of NEMO/IKKgamma by SUMO-1 and ubiquitin mediates NF-kappaB activation by genotoxic stress. Cell 115, 565-576.

Imin, N., Kerim, T., Rolfe, B.G., and Weinman, J.J. (2004). Effect of early cold stress on the maturation of rice anthers. Proteomics 4, 1873-1882.

Iniguez-Lluhi, J.A. (2006). For a healthy histone code, a little SUMO in the tail keeps the acetyl away. ACS Chem Biol 1, 204-206.

Ise, T., Nagatani, G., Imamura, T., Kato, K., Takano, H., Nomoto, M., Izumi, H., Ohmori, H., Okamoto, T., Ohga, T., Uchiumi, T., Kuwano, M., and Kohno, K. (1999). Transcription factor Y-box binding protein 1 binds preferentially to cisplatin-modified DNA and interacts with proliferating cell nuclear antigen. Cancer Res 59, 342-346.

Ishov, A.M., Sotnikov, A.G., Negorev, D., Vladimirova, O.V., Neff, N., Kamitani, T., Yeh, E.T., Strauss, J.F., 3rd, and Maul, G.G. (1999). PML is critical for ND10 formation and recruits the PML-interacting protein daxx to this nuclear structure when modified by SUMO-1. J Cell Biol 147, 221-234.

Ishov, A.M., Vladimirova, O.V., and Maul, G.G. (2002). Daxx-mediated accumulation of human cytomegalovirus tegument protein pp71 at ND10 facilitates initiation of viral infection at these nuclear domains. J Virol 76, 7705-7712.

Jackson, P.K. (2001). A new RING for SUMO: wrestling transcriptional responses into nuclear bodies with PIAS family E3 SUMO ligases. Genes Dev 15, 3053-3058.

Jiang, W., Fang, L., and Inouye, M. (1996). Complete growth inhibition of Escherichia coli by ribosome trapping with truncated cspA mRNA at low temperature. Genes Cells 1, 965-976.

Jiang, W., Fang, L., and Inouye, M. (1996). The role of the 5'-end untranslated region of the mRNA for CspA, the major cold-shock protein of Escherichia coli, in coldshock adaptation. J Bacteriol 178, 4919-4925.

Jiang, W., Hou, Y., and Inouye, M. (1997). CspA, the major cold-shock protein of Escherichia coli, is an RNA chaperone. J Biol Chem 272, 196-202.

Jin, C., Shiyanova, T., Shen, Z., and Liao, X. (2001). Heteronuclear nuclear magnetic resonance assignments, structure and dynamics of SUMO-1, a human ubiquitinlike protein. Int J Biol Macromol 28, 227-234.

Jin, J.B., Jin, Y.H., Lee, J., Miura, K., Yoo, C.Y., Kim, W.Y., Van Oosten, M., Hyun, Y., Somers, D.E., Lee, I., Yun, D.J., Bressan, R.A., and Hasegawa, P.M. (2007). The SUMO E3 ligase, AtSIZ1, regulates flowering by controlling a salicylic acid-mediated floral promotion pathway and through affects on FLC chromatin structure. Plant J. 
Jin, J.B., Jin, Y.H., Lee, J., Miura, K., Yoo, C.Y., Kim, W.Y., Van Oosten, M., Hyun, Y., Somers, D.E., Lee, I., Yun, D.J., Bressan, R.A., and Hasegawa, P.M. (2008). The SUMO E3 ligase, AtSIZ1, regulates flowering by controlling a salicylic acid-mediated floral promotion pathway and through affects on FLC chromatin structure. Plant J 53, 530-540.

Johnson, E.S., and Blobel, G. (1997). Ubc9p is the conjugating enzyme for the ubiquitin-like protein Smt3p. J Biol Chem 272, 26799-26802.

Johnson, E.S., Schwienhorst, I., Dohmen, R.J., and Blobel, G. (1997). The ubiquitinlike protein Smt $3 p$ is activated for conjugation to other proteins by an Aos1p/Uba2p heterodimer. Embo J 16, 5509-5519.

Johnson, E.S., and Gupta, A.A. (2001). An E3-like factor that promotes SUMO conjugation to the yeast septins. Cell 106, 735-744.

Johnson, E.S. (2004). Protein modification by SUMO. Annu Rev Biochem 73, 355-382.

Jones, M.C., Fusi, L., Higham, J.H., Abdel-Hafiz, H., Horwitz, K.B., Lam, E.W., and Brosens, J.J. (2006). Regulation of the SUMO pathway sensitizes differentiating human endometrial stromal cells to progesterone. Proc Natl Acad Sci USA 103, 16272-16277.

Joseph, J., Tan, S.H., Karpova, T.S., McNally, J.G., and Dasso, M. (2002). SUMO-1 targets RanGAP1 to kinetochores and mitotic spindles. J Cell Biol 156, 595-602.

Joseph, J., Liu, S.T., Jablonski, S.A., Yen, T.J., and Dasso, M. (2004). The RanGAP1RanBP2 complex is essential for microtubule-kinetochore interactions in vivo. Curr Biol 14, 611-617.

Jurchott, K., Bergmann, S., Stein, U., Walther, W., Janz, M., Manni, I., Piaggio, G., Fietze, E., Dietel, M., and Royer, H.D. (2003). YB-1 as a cell cycle-regulated transcription factor facilitating cyclin A and cyclin B1 gene expression. J Biol Chem 278, 27988-27996.

Kagey, M.H., Melhuish, T.A., and Wotton, D. (2003). The polycomb protein Pc2 is a SUMO E3. Cell 113, 127-137.

Kahyo, T., Nishida, T., and Yasuda, H. (2001). Involvement of PIAS1 in the sumoylation of tumor suppressor p53. Mol Cell 8, 713-718.

Kang, J., Gocke, C.B., and Yu, H. (2006). Phosphorylation-facilitated sumoylation of MEF2C negatively regulates its transcriptional activity. BMC Biochem 7, 5.

Karlson, D., Nakaminami, K., Toyomasu, T., and Imai, R. (2002). A cold-regulated nucleic acid-binding protein of winter wheat shares a domain with bacterial cold shock proteins. J Biol Chem 277, 35248-35256.

Karlson, D., and Imai, R. (2003). Conservation of the cold shock domain protein family in plants. Plant Physiol 131, 12-15.

Karlson, D.T., Zeng, Y., Stirm, V.E., Joly, R.J., and Ashworth, E.N. (2003). Photoperiodic regulation of a $24-\mathrm{kD}$ dehydrin-like protein in red-osier dogwood (Cornus sericea L.) in relation to freeze-tolerance. Plant Cell Physiol 44, 25-34.

Kazemitabar, S.K., Tomsett, A.B., Collin, H.A., Wilkinson, M.C., and Jones, M.G. (2003). Effect of short term cold stress on rice seedlings. Euphytica 129, 193-200.

Kim, J.S., Park, S.J., Kwak, K.J., Kim, Y.O., Kim, J.Y., Song, J., Jang, B., Jung, C.H., and Kang, H. (2007). Cold shock domain proteins and glycine-rich RNAbinding proteins from Arabidopsis thaliana can promote the cold adaptation process in Escherichia coli. Nucleic Acids Res 35, 506-516. 
Kingsley, P.D., and Palis, J. (1994). GRP2 proteins contain both CCHC zinc fingers and a cold shock domain. Plant Cell 6, 1522-1523.

Kloks, C.P., Spronk, C.A., Lasonder, E., Hoffmann, A., Vuister, G.W., Grzesiek, S., and Hilbers, C.W. (2002). The solution structure and DNA-binding properties of the cold-shock domain of the human Y-box protein YB-1. J Mol Biol 316, 317326.

Kohno, K., Izumi, H., Uchiumi, T., Ashizuka, M., and Kuwano, M. (2003). The pleiotropic functions of the Y-box-binding protein, YB-1. Bioessays 25, 691-698.

Koike, K., Uchiumi, T., Ohga, T., Toh, S., Wada, M., Kohno, K., and Kuwano, M. (1997). Nuclear translocation of the Y-box binding protein by ultraviolet irradiation. FEBS Lett 417, 390-394.

Kurepa, J., Walker, J.M., Smalle, J., Gosink, M.M., Davis, S.J., Durham, T.L., Sung, D.Y., and Vierstra, R.D. (2003). The small ubiquitin-like modifier (SUMO) protein modification system in Arabidopsis. Accumulation of SUMO1 and -2 conjugates is increased by stress. J Biol Chem 278, 6862-6872.

Landsman, D. (1992). RNP-1, an RNA-binding motif is conserved in the DNA-binding cold shock domain. Nucleic Acids Res 20, 2861-2864.

Larkin, M.A., Blackshields, G., Brown, N.P., Chenna, R., McGettigan, P.A., McWilliam, H., Valentin, F., Wallace, I.M., Wilm, A., Lopez, R., Thompson, J.D., Gibson, T.J., and Higgins, D.G. (2007). Clustal W and Clustal X version 2.0. Bioinformatics 23, 2947-2948.

Lee, S.J., Xie, A., Jiang, W., Etchegaray, J.P., Jones, P.G., and Inouye, M. (1994). Family of the major cold-shock protein, CspA (CS7.4), of Escherichia coli, whose members show a high sequence similarity with the eukaryotic Y-box binding proteins. Mol Microbiol 11, 833-839.

Lee, J., Nam, J., Park, H.C., Na, G., Miura, K., Jin, J.B., Yoo, C.Y., Baek, D., Kim, D.H., Jeong, J.C., Kim, D., Lee, S.Y., Salt, D.E., Mengiste, T., Gong, Q., Ma, S., Bohnert, H.J., Kwak, S.S., Bressan, R.A., Hasegawa, P.M., and Yun, D.J. (2007). Salicylic acid-mediated innate immunity in Arabidopsis is regulated by SIZ1 SUMO E3 ligase. Plant J 49, 79-90.

Levenson, V.V., Davidovich, I.A., and Roninson, I.B. (2000). Pleiotropic resistance to DNA-interactive drugs is associated with increased expression of genes involved in DNA replication, repair, and stress response. Cancer Res 60, 5027-5030.

Li, S.J., and Hochstrasser, M. (1999). A new protease required for cell-cycle progression in yeast. Nature 398, 246-251.

Lin, D., Tatham, M.H., Yu, B., Kim, S., Hay, R.T., and Chen, Y. (2002). Identification of a substrate recognition site on Ubc9. J Biol Chem 277, 2174021748.

Lin, X., Liang, M., Liang, Y.Y., Brunicardi, F.C., and Feng, X.H. (2003). SUMO$1 /$ Ubc9 promotes nuclear accumulation and metabolic stability of tumor suppressor Smad4. J Biol Chem 278, 31043-31048.

Lin, J.Y., Ohshima, T., and Shimotohno, K. (2004). Association of Ubc9, an E2 ligase for SUMO conjugation, with p53 is regulated by phosphorylation of p53. FEBS Lett 573, 15-18.

Lin, D.Y., Huang, Y.S., Jeng, J.C., Kuo, H.Y., Chang, C.C., Chao, T.T., Ho, C.C., Chen, Y.C., Lin, T.P., Fang, H.I., Hung, C.C., Suen, C.S., Hwang, M.J., 
Chang, K.S., Maul, G.G., and Shih, H.M. (2006). Role of SUMO-interacting motif in Daxx SUMO modification, subnuclear localization, and repression of sumoylated transcription factors. Mol Cell 24, 341-354.

Liu, B., and Shuai, K. (2008). Regulation of the sumoylation system in gene expression. Curr Opin Cell Biol 20, 288-293.

Lois, L.M., Lima, C.D., and Chua, N.H. (2003). Small ubiquitin-like modifier modulates abscisic acid signaling in Arabidopsis. Plant Cell 15, 1347-1359.

Lu, Z.H., Books, J.T., and Ley, T.J. (2005). YB-1 is important for late-stage embryonic development, optimal cellular stress responses, and the prevention of premature senescence. Mol Cell Biol 25, 4625-4637.

Lu, Z.H., Books, J.T., and Ley, T.J. (2006). Cold shock domain family members YB-1 and MSY4 share essential functions during murine embryogenesis. Mol Cell Biol 26, 8410-8417.

Lyst, M.J., and Stancheva, I. (2007). A role for SUMO modification in transcriptional repression and activation. Biochem Soc Trans 35, 1389-1392.

Mabb, A.M., Wuerzberger-Davis, S.M., and Miyamoto, S. (2006). PIASy mediates NEMO sumoylation and NF-kappaB activation in response to genotoxic stress. Nat Cell Biol 8, 986-993.

Mahajan, R., Delphin, C., Guan, T., Gerace, L., and Melchior, F. (1997). A small ubiquitin-related polypeptide involved in targeting RanGAP1 to nuclear pore complex protein RanBP2. Cell 88, 97-107.

Mahajan, S., and Tuteja, N. (2005). Cold, salinity and drought stresses: an overview. Arch Biochem Biophys 444, 139-158.

Manival, X., Ghisolfi-Nieto, L., Joseph, G., Bouvet, P., and Erard, M. (2001). RNAbinding strategies common to cold-shock domain- and RNA recognition motifcontaining proteins. Nucleic Acids Res 29, 2223-2233.

Matsumoto, K., and Wolffe, A.P. (1998). Gene regulation by Y-box proteins: coupling control of transcription and translation. Trends Cell Biol 8, 318-323.

Matsumoto, K., Tanaka, K.J., and Tsujimoto, M. (2005). An acidic protein, YBAP1, mediates the release of YB-1 from mRNA and relieves the translational repression activity of YB-1. Mol Cell Biol 25, 1779-1792.

Matunis, M.J., Coutavas, E., and Blobel, G. (1996). A novel ubiquitin-like modification modulates the partitioning of the Ran-GTPase-activating protein RanGAP1 between the cytosol and the nuclear pore complex. J Cell Biol 135, 1457-1470.

Matunis, M.J., Wu, J., and Blobel, G. (1998). SUMO-1 modification and its role in targeting the Ran GTPase-activating protein, RanGAP1, to the nuclear pore complex. J Cell Biol 140, 499-509.

Mayer, R.J., Landon, M., and Layfield, R. (1998). Ubiquitin superfolds: intrinsic and attachable regulators of cellular activities? Fold Des 3, R97-99.

Melchior, F. (2000). SUMO--nonclassical ubiquitin. Annu Rev Cell Dev Biol 16, 591626.

Melchior, F., Schergaut, M., and Pichler, A. (2003). SUMO: ligases, isopeptidases and nuclear pores. Trends Biochem Sci 28, 612-618.

Meluh, P.B., and Koshland, D. (1995). Evidence that the MIF2 gene of Saccharomyces cerevisiae encodes a centromere protein with homology to the mammalian 
centromere protein CENP-C. Mol Biol Cell 6, 793-807.

Mertens, P.R., Harendza, S., Pollock, A.S., and Lovett, D.H. (1997). Glomerular mesangial cell-specific transactivation of matrix metalloproteinase 2 transcription is mediated by YB-1. J Biol Chem 272, 22905-22912.

Minich, W.B., and Ovchinnikov, L.P. (1992). Role of cytoplasmic mRNP proteins in translation. Biochimie 74, 477-483.

Minich, W.B., Maidebura, I.P., and Ovchinnikov, L.P. (1993). Purification and characterization of the major $50-\mathrm{kDa}$ repressor protein from cytoplasmic mRNP of rabbit reticulocytes. Eur J Biochem 212, 633-638.

Minty, A., Dumont, X., Kaghad, M., and Caput, D. (2000). Covalent modification of p73alpha by SUMO-1. Two-hybrid screening with p73 identifies novel SUMO-1interacting proteins and a SUMO-1 interaction motif. J Biol Chem 275, 3631636323.

Miura, K., Rus, A., Sharkhuu, A., Yokoi, S., Karthikeyan, A.S., Raghothama, K.G., Baek, D., Koo, Y.D., Jin, J.B., Bressan, R.A., Yun, D.J., and Hasegawa, P.M. (2005). The Arabidopsis SUMO E3 ligase SIZ1 controls phosphate deficiency responses. Proc Natl Acad Sci USA 102, 7760-7765.

Miura, K., Jin, J.B., and Hasegawa, P.M. (2007). Sumoylation, a post-translational regulatory process in plants. Curr Opin Plant Biol 10, 495-502.

Miura, K., Jin, J.B., Lee, J., Yoo, C.Y., Stirm, V., Miura, T., Ashworth, E.N., Bressan, R.A., Yun, D.J., and Hasegawa, P.M. (2007). SIZ1-mediated sumoylation of ICE1 controls CBF3/DREB1A expression and freezing tolerance in Arabidopsis. Plant Cell 19, 1403-1414.

Miura, K., Lee, J., Jin, J.B., Yoo, C.Y., Miura, T., and Hasegawa, P.M. (2009). Sumoylation of ABI5 by the Arabidopsis SUMO E3 ligase SIZ1 negatively regulates abscisic acid signaling. Proc Natl Acad Sci USA.

Moss, E.G., Lee, R.C., and Ambros, V. (1997). The cold shock domain protein LIN-28 controls developmental timing in $\mathrm{C}$. elegans and is regulated by the lin-4 RNA. Cell 88, 637-646.

Mossessova, E., and Lima, C.D. (2000). Ulp1-SUMO crystal structure and genetic analysis reveal conserved interactions and a regulatory element essential for cell growth in yeast. Mol Cell 5, 865-876.

Muller, S., Berger, M., Lehembre, F., Seeler, J.S., Haupt, Y., and Dejean, A. (2000). c-Jun and p53 activity is modulated by SUMO-1 modification. J Biol Chem 275, 13321-13329.

Muller, S., Hoege, C., Pyrowolakis, G., and Jentsch, S. (2001). SUMO, ubiquitin's mysterious cousin. Nat Rev Mol Cell Biol 2, 202-210.

Muller, S., Ledl, A., and Schmidt, D. (2004). SUMO: a regulator of gene expression and genome integrity. Oncogene 23, 1998-2008.

Murray, M.T., Krohne, G., and Franke, W.W. (1991). Different forms of soluble cytoplasmic mRNA binding proteins and particles in Xenopus laevis oocytes and embryos. J Cell Biol 112, 1-11.

Murtas, G., Reeves, P.H., Fu, Y.F., Bancroft, I., Dean, C., and Coupland, G. (2003). A nuclear protease required for flowering-time regulation in Arabidopsis reduces the abundance of SMALL UBIQUITIN-RELATED MODIFIER conjugates. Plant Cell 15, 2308-2319. 
Mussgnug, J.H., Wobbe, L., Elles, I., Claus, C., Hamilton, M., Fink, A., Kahmann, U., Kapazoglou, A., Mullineaux, C.W., Hippler, M., Nickelsen, J., Nixon, P.J., and Kruse, O. (2005). NAB1 is an RNA binding protein involved in the lightregulated differential expression of the light-harvesting antenna of Chlamydomonas reinhardtii. Plant Cell 17, 3409-3421.

Nakaminami, K., Karlson, D.T., and Imai, R. (2006). Functional conservation of cold shock domains in bacteria and higher plants. Proc Natl Acad Sci USA 103, 10122-10127.

Nakaminami, K., Hill, K., Perry, S.E., Sentoku, N., Long, J.A., and Karlson, D.T. (2009). Arabidopsis cold shock domain proteins: relationships to floral and silique development. J Exp Bot 60, 1047-1062.

Nakashima, K., Kanamaru, K., Mizuno, T., and Horikoshi, K. (1996). A novel member of the cspA family of genes that is induced by cold shock in Escherichia coli. J Bacteriol 178, 2994-2997.

Nathan, D., Sterner, D.E., and Berger, S.L. (2003). Histone modifications: Now summoning sumoylation. Proc Natl Acad Sci USA 100, 13118-13120.

Nathan, D., Ingvarsdottir, K., Sterner, D.E., Bylebyl, G.R., Dokmanovic, M., Dorsey, J.A., Whelan, K.A., Krsmanovic, M., Lane, W.S., Meluh, P.B., Johnson, E.S., and Berger, S.L. (2006). Histone sumoylation is a negative regulator in Saccharomyces cerevisiae and shows dynamic interplay with positive-acting histone modifications. Genes Dev 20, 966-976.

Nekrasov, M.P., Ivshina, M.P., Chernov, K.G., Kovrigina, E.A., Evdokimova, V.M., Thomas, A.A., Hershey, J.W., and Ovchinnikov, L.P. (2003). The mRNAbinding protein YB-1 (p50) prevents association of the eukaryotic initiation factor eIF4G with mRNA and inhibits protein synthesis at the initiation stage. $J$ Biol Chem 278, 13936-13943.

Newkirk, K., Feng, W., Jiang, W., Tejero, R., Emerson, S.D., Inouye, M., and Montelione, G.T. (1994). Solution NMR structure of the major cold shock protein (CspA) from Escherichia coli: identification of a binding epitope for DNA. Proc Natl Acad Sci USA 91, 5114-5118.

Niwa, Y., Hirano, T., Yoshimoto, K., Shimizu, M., and Kobayashi, H. (1999). Noninvasive quantitative detection and applications of non-toxic, S65T-type green fluorescent protein in living plants. Plant J 18, 455-463.

Novatchkova, M., Budhiraja, R., Coupland, G., Eisenhaber, F., and Bachmair, A. (2004). SUMO conjugation in plants. Planta 220, 1-8.

Okubo, S., Hara, F., Tsuchida, Y., Shimotakahara, S., Suzuki, S., Hatanaka, H., Yokoyama, S., Tanaka, H., Yasuda, H., and Shindo, H. (2004). NMR structure of the N-terminal domain of SUMO ligase PIAS1 and its interaction with tumor suppressor p53 and A/T-rich DNA oligomers. J Biol Chem 279, 31455-31461.

Okuma, T., Honda, R., Ichikawa, G., Tsumagari, N., and Yasuda, H. (1999). In vitro SUMO-1 modification requires two enzymatic steps, E1 and E2. Biochem Biophys Res Commun 254, 693-698.

Palvimo, J.J. (2007). PIAS proteins as regulators of small ubiquitin-related modifier (SUMO) modifications and transcription. Biochem Soc Trans 35, 1405-1408.

Papouli, E., Chen, S., Davies, A.A., Huttner, D., Krejci, L., Sung, P., and Ulrich, H.D. (2005). Crosstalk between SUMO and ubiquitin on PCNA is mediated by 
recruitment of the helicase Srs2p. Mol Cell 19, 123-133.

Pastushok, L., and Xiao, W. (2004). DNA postreplication repair modulated by ubiquitination and sumoylation. Adv Protein Chem 69, 279-306.

Phadtare, S., and Inouye, M. (1999). Sequence-selective interactions with RNA by CspB, CspC and CspE, members of the CspA family of Escherichia coli. Mol Microbiol 33, 1004-1014.

Phadtare, S., and Inouye, M. (2001). Role of $\mathrm{CspC}$ and $\mathrm{CspE}$ in regulation of expression of RpoS and UspA, the stress response proteins in Escherichia coli. J Bacteriol 183, 1205-1214.

Phadtare, S., Inouye, M., and Severinov, K. (2002). The nucleic acid melting activity of Escherichia coli $\mathrm{CspE}$ is critical for transcription antitermination and cold acclimation of cells. J Biol Chem 277, 7239-7245.

Phadtare, S., and Severinov, K. (2005). Nucleic acid melting by Escherichia coli CspE. Nucleic Acids Res 33, 5583-5590.

Pichler, A., Gast, A., Seeler, J.S., Dejean, A., and Melchior, F. (2002). The nucleoporin RanBP2 has SUMO1 E3 ligase activity. Cell 108, 109-120.

Pichler, A., and Melchior, F. (2002). Ubiquitin-related modifier SUMO1 and nucleocytoplasmic transport. Traffic 3, 381-387.

Pichler, A., Knipscheer, P., Oberhofer, E., van Dijk, W.J., Korner, R., Olsen, J.V., Jentsch, S., Melchior, F., and Sixma, T.K. (2005). SUMO modification of the ubiquitin-conjugating enzyme E2-25K. Nat Struct Mol Biol 12, 264-269.

Preobrazhensky, A.A., and Spirin, A.S. (1978). Informosomes and their protein components: the present state of knowledge. Prog Nucleic Acid Res Mol Biol 21, $1-38$.

Prudden, J., Pebernard, S., Raffa, G., Slavin, D.A., Perry, J.J., Tainer, J.A., McGowan, C.H., and Boddy, M.N. (2007). SUMO-targeted ubiquitin ligases in genome stability. Embo J 26, 4089-4101.

Rabbani, M.A., Maruyama, K., Abe, H., Khan, M.A., Katsura, K., Ito, Y., Yoshiwara, K., Seki, M., Shinozaki, K., and Yamaguchi-Shinozaki, K. (2003). Monitoring expression profiles of rice genes under cold, drought, and highsalinity stresses and abscisic acid application using cDNA microarray and RNA gel-blot analyses. Plant Physiol 133, 1755-1767.

Rajavashisth, T.B., Taylor, A.K., Andalibi, A., Svenson, K.L., and Lusis, A.J. (1989). Identification of a zinc finger protein that binds to the sterol regulatory element. Science 245, 640-643.

Reverter, D., and Lima, C.D. (2005). Insights into E3 ligase activity revealed by a SUMO-RanGAP1-Ubc9-Nup358 complex. Nature 435, 687-692.

Roden, J., Eardley, L., Hotson, A., Cao, Y., and Mudgett, M.B. (2004). Characterization of the Xanthomonas AvrXv4 effector, a SUMO protease translocated into plant cells. Mol Plant Microbe Interact 17, 633-643.

Rodriguez, M.S., Dargemont, C., and Hay, R.T. (2001). SUMO-1 conjugation in vivo requires both a consensus modification motif and nuclear targeting. J Biol Chem 276, 12654-12659.

Roscic, A., Moller, A., Calzado, M.A., Renner, F., Wimmer, V.C., Gresko, E., Ludi, K.S., and Schmitz, M.L. (2006). Phosphorylation-dependent control of Pc2 SUMO E3 ligase activity by its substrate protein HIPK2. Mol Cell 24, 77-89. 
Rose, A., and Meier, I. (2001). A domain unique to plant RanGAP is responsible for its targeting to the plant nuclear rim. Proc Natl Acad Sci USA 98, 15377-15382.

Roussell, D.L., and Bennett, K.L. (1993). glh-1, a germ-line putative RNA helicase from Caenorhabditis, has four zinc fingers. Proc Natl Acad Sci USA 90, 93009304.

Sachdev, S., Bruhn, L., Sieber, H., Pichler, A., Melchior, F., and Grosschedl, R. (2001). PIASy, a nuclear matrix-associated SUMO E3 ligase, represses LEF1 activity by sequestration into nuclear bodies. Genes Dev 15, 3088-3103.

Sacher, M., Pfander, B., Hoege, C., and Jentsch, S. (2006). Control of Rad52 recombination activity by double-strand break-induced SUMO modification. Nat Cell Biol 8, 1284-1290.

Saitoh, H., and Hinchey, J. (2000). Functional heterogeneity of small ubiquitin-related protein modifiers SUMO-1 versus SUMO-2/3. J Biol Chem 275, 6252-6258.

Sampson, D.A., Wang, M., and Matunis, M.J. (2001). The small ubiquitin-like modifier-1 (SUMO-1) consensus sequence mediates Ubc9 binding and is essential for SUMO-1 modification. J Biol Chem 276, 21664-21669.

Sapetschnig, A., Rischitor, G., Braun, H., Doll, A., Schergaut, M., Melchior, F., and Suske, G. (2002). Transcription factor Sp3 is silenced through SUMO modification by PIAS1. Embo J 21, 5206-5215.

Saracco, S.A., Miller, M.J., Kurepa, J., and Vierstra, R.D. (2007). Genetic analysis of SUMOylation in Arabidopsis: conjugation of SUMO1 and SUMO2 to nuclear proteins is essential. Plant Physiol 145, 119-134.

Sasaki, K., Kim, M.H., and Imai, R. (2007). Arabidopsis COLD SHOCK DOMAIN PROTEIN2 is a RNA chaperone that is regulated by cold and developmental signals. Biochem Biophys Res Commun 364, 633-638.

Schindelin, H., Marahiel, M.A., and Heinemann, U. (1993). Universal nucleic acidbinding domain revealed by crystal structure of the B. subtilis major cold-shock protein. Nature 364, 164-168.

Schindelin, H., Jiang, W., Inouye, M., and Heinemann, U. (1994). Crystal structure of CspA, the major cold shock protein of Escherichia coli. Proc Natl Acad Sci USA 91, 5119-5123.

Schmidt, D., and Muller, S. (2002). Members of the PIAS family act as SUMO ligases for c-Jun and p53 and repress p53 activity. Proc Natl Acad Sci USA 99, 28722877.

Schnuchel, A., Wiltscheck, R., Czisch, M., Herrler, M., Willimsky, G., Graumann, P., Marahiel, M.A., and Holak, T.A. (1993). Structure in solution of the major cold-shock protein from Bacillus subtilis. Nature 364, 169-171.

Schroder, K., Graumann, P., Schnuchel, A., Holak, T.A., and Marahiel, M.A. (1995). Mutational analysis of the putative nucleic acid-binding surface of the cold-shock domain, $\mathrm{CspB}$, revealed an essential role of aromatic and basic residues in binding of single-stranded DNA containing the Y-box motif. Mol Microbiol 16, 699-708.

Seeler, J.S., and Dejean, A. (2003). Nuclear and unclear functions of SUMO. Nat Rev Mol Cell Biol 4, 690-699.

Sharrocks, A.D. (2006). PIAS proteins and transcriptional regulation--more than just SUMO E3 ligases? Genes Dev 20, 754-758. 
Shen, T.H., Lin, H.K., Scaglioni, P.P., Yung, T.M., and Pandolfi, P.P. (2006). The mechanisms of PML-nuclear body formation. Mol Cell 24, 331-339.

Shiio, Y., and Eisenman, R.N. (2003). Histone sumoylation is associated with transcriptional repression. Proc Natl Acad Sci USA 100, 13225-13230.

Skabkin, M.A., Evdokimova, V., Thomas, A.A., and Ovchinnikov, L.P. (2001). The major messenger ribonucleoprotein particle protein p50 (YB-1) promotes nucleic acid strand annealing. J Biol Chem 276, 44841-44847.

Skabkin, M.A., Liabin, D.N., and Ovchinnikov, L.P. (2006). [Nonspecific and specific interaction of Y-box binding protein 1 (YB-1) with mRNA and posttranscriptional regulation of protein synthesis in animal cells]. Mol Biol (Mosk) 40, 620-633.

Skabkina, O.V., Skabkin, M.A., Popova, N.V., Lyabin, D.N., Penalva, L.O., and Ovchinnikov, L.P. (2003). Poly(A)-binding protein positively affects YB-1 mRNA translation through specific interaction with YB-1 mRNA. J Biol Chem 278, 18191-18198.

Skabkina, O.V., Lyabin, D.N., Skabkin, M.A., and Ovchinnikov, L.P. (2005). YB-1 autoregulates translation of its own mRNA at or prior to the step of $40 \mathrm{~S}$ ribosomal subunit joining. Mol Cell Biol 25, 3317-3323.

Sobko, A., Ma, H., and Firtel, R.A. (2002). Regulated SUMOylation and ubiquitination of DdMEK1 is required for proper chemotaxis. Dev Cell 2, 745-756.

Sommerville, J., and Ladomery, M. (1996). Masking of mRNA by Y-box proteins. FASEB J 10, 435-443.

Sommerville, J. (1999). Activities of cold-shock domain proteins in translation control. Bioessays 21, 319-325.

Song, J., Durrin, L.K., Wilkinson, T.A., Krontiris, T.G., and Chen, Y. (2004). Identification of a SUMO-binding motif that recognizes SUMO-modified proteins. Proc Natl Acad Sci USA 101, 14373-14378.

Soop, T., Nashchekin, D., Zhao, J., Sun, X., Alzhanova-Ericsson, A.T., Bjorkroth, B., Ovchinnikov, L., and Daneholt, B. (2003). A p50-like Y-box protein with a putative translational role becomes associated with pre-mRNA concomitant with transcription. J Cell Sci 116, 1493-1503.

Stead, K., Aguilar, C., Hartman, T., Drexel, M., Meluh, P., and Guacci, V. (2003). Pds5p regulates the maintenance of sister chromatid cohesion and is sumoylated to promote the dissolution of cohesion. J Cell Biol 163, 729-741.

Steffan, J.S., Agrawal, N., Pallos, J., Rockabrand, E., Trotman, L.C., Slepko, N., Illes, K., Lukacsovich, T., Zhu, Y.Z., Cattaneo, E., Pandolfi, P.P., Thompson, L.M., and Marsh, J.L. (2004). SUMO modification of Huntingtin and Huntington's disease pathology. Science 304, 100-104.

Stenina, O.I., Shaneyfelt, K.M., and DiCorleto, P.E. (2001). Thrombin induces the release of the Y-box protein dbpB from mRNA: a mechanism of transcriptional activation. Proc Natl Acad Sci USA 98, 7277-7282.

Sternsdorf, T., Jensen, K., and Will, H. (1997). Evidence for covalent modification of the nuclear dot-associated proteins PML and Sp100 by PIC1/SUMO-1. J Cell Biol 139, 1621-1634.

Sternsdorf, T., Jensen, K., Reich, B., and Will, H. (1999). The nuclear dot protein sp100, characterization of domains necessary for dimerization, subcellular localization, and modification by small ubiquitin-like modifiers. J Biol Chem 274, 
12555-12566.

Stickeler, E., Fraser, S.D., Honig, A., Chen, A.L., Berget, S.M., and Cooper, T.A. (2001). The RNA binding protein YB-1 binds A/C-rich exon enhancers and stimulates splicing of the CD44 alternative exon v4. EMBO J 20, 3821-3830.

Sun, H., Leverson, J.D., and Hunter, T. (2007). Conserved function of RNF4 family proteins in eukaryotes: targeting a ubiquitin ligase to SUMOylated proteins. Embo J 26, 4102-4112.

Sutherland, B.W., Kucab, J., Wu, J., Lee, C., Cheang, M.C., Yorida, E., Turbin, D., Dedhar, S., Nelson, C., Pollak, M., Leighton Grimes, H., Miller, K., Badve, S., Huntsman, D., Blake-Gilks, C., Chen, M., Pallen, C.J., and Dunn, S.E. (2005). Akt phosphorylates the Y-box binding protein 1 at Ser102 located in the cold shock domain and affects the anchorage-independent growth of breast cancer cells. Oncogene 24, 4281-4292.

Swamynathan, S.K., Nambiar, A., and Guntaka, R.V. (1998). Role of single-stranded DNA regions and Y-box proteins in transcriptional regulation of viral and cellular genes. FASEB J 12, 515-522.

Tafuri, S.R., and Wolffe, A.P. (1993). Selective recruitment of masked maternal mRNA from messenger ribonucleoprotein particles containing FRGY2 (mRNP4). J Biol Chem 268, 24255-24261.

Takahashi, Y., Toh-e, A., and Kikuchi, Y. (2001). A novel factor required for the SUMO1/Smt3 conjugation of yeast septins. Gene 275, 223-231.

Tanabe, H., Goldstein, J., Yang, M., and Inouye, M. (1992). Identification of the promoter region of the Escherichia coli major cold shock gene, cspA. J Bacteriol 174, 3867-3873.

Tanaka, K., Nishide, J., Okazaki, K., Kato, H., Niwa, O., Nakagawa, T., Matsuda, H., Kawamukai, M., and Murakami, Y. (1999). Characterization of a fission yeast SUMO-1 homologue, pmt3p, required for multiple nuclear events, including the control of telomere length and chromosome segregation. Mol Cell Biol 19, 8660-8672.

Tang, Z., Hecker, C.M., Scheschonka, A., and Betz, H. (2008). Protein interactions in the sumoylation cascade: lessons from X-ray structures. Febs J 275, 3003-3015.

Tatham, M.H., Jaffray, E., Vaughan, O.A., Desterro, J.M., Botting, C.H., Naismith, J.H., and Hay, R.T. (2001). Polymeric chains of SUMO-2 and SUMO-3 are conjugated to protein substrates by SAE1/SAE2 and Ubc9. J Biol Chem 276, 35368-35374.

Tatham, M.H., Chen, Y., and Hay, R.T. (2003). Role of two residues proximal to the active site of Ubc9 in substrate recognition by the Ubc9.SUMO-1 thiolester complex. Biochemistry 42, 3168-3179.

Tempe, D., Piechaczyk, M., and Bossis, G. (2008). SUMO under stress. Biochem Soc Trans 36, 874-878.

Terui, Y., Saad, N., Jia, S., McKeon, F., and Yuan, J. (2004). Dual role of sumoylation in the nuclear localization and transcriptional activation of NFAT1. J Biol Chem 279, 28257-28265.

Thomashow, M.F. (1999). PLANT COLD ACCLIMATION: Freezing Tolerance Genes and Regulatory Mechanisms. Annu Rev Plant Physiol Plant Mol Biol 50, 571599. 
Uchiumi, T., Fotovati, A., Sasaguri, T., Shibahara, K., Shimada, T., Fukuda, T., Nakamura, T., Izumi, H., Tsuzuki, T., Kuwano, M., and Kohno, K. (2006). YB-1 is important for an early stage embryonic development: neural tube formation and cell proliferation. J Biol Chem 281, 40440-40449.

Uzunova, K., Gottsche, K., Miteva, M., Weisshaar, S.R., Glanemann, C., Schnellhardt, M., Niessen, M., Scheel, H., Hofmann, K., Johnson, E.S., Praefcke, G.J., and Dohmen, R.J. (2007). Ubiquitin-dependent proteolytic control of SUMO conjugates. J Biol Chem 282, 34167-34175.

van Venrooij, W.J., van Eekelen, C.A., Jansen, R.T., and Princen, J.M. (1977). Specific poly-A-binding protein of 76,000 molecular weight in polyribosomes is not present on poly A of free cytoplasmic mRNP. Nature 270, 189-191.

Verger, A., Perdomo, J., and Crossley, M. (2003). Modification with SUMO. A role in transcriptional regulation. EMBO Rep 4, 137-142.

Viswanathan, C., and Zhu, J.K. (2002). Molecular genetic analysis of cold-regulated gene transcription. Philos Trans R Soc Lond B Biol Sci 357, 877-886.

Wang, N., Yamanaka, K., and Inouye, M. (1999). CspI, the ninth member of the CspA family of Escherichia coli, is induced upon cold shock. J Bacteriol 181, 16031609.

Watts, F.Z. (2006). Sumoylation of PCNA: Wrestling with recombination at stalled replication forks. DNA Repair (Amst) 5, 399-403.

Weissman, A.M. (2001). Themes and variations on ubiquitylation. Nat Rev Mol Cell Biol 2, 169-178.

Wen, J.Q., Oono, K., and Imai, R. (2002). Two novel mitogen-activated protein signaling components, OsMEK1 and OsMAP1, are involved in a moderate lowtemperature signaling pathway in rice. Plant Physiol 129, 1880-1891.

Wolffe, A.P., Tafuri, S., Ranjan, M., and Familari, M. (1992). The Y-box factors: a family of nucleic acid binding proteins conserved from Escherichia coli to man. New Biol 4, 290-298.

Wolffe, A.P. (1994). Structural and functional properties of the evolutionarily ancient Ybox family of nucleic acid binding proteins. Bioessays 16, 245-251.

Wood, L.D., Irvin, B.J., Nucifora, G., Luce, K.S., and Hiebert, S.W. (2003). Small ubiquitin-like modifier conjugation regulates nuclear export of TEL, a putative tumor suppressor. Proc Natl Acad Sci USA 100, 3257-3262.

Wotton, D., and Merrill, J.C. (2007). Pc2 and SUMOylation. Biochem Soc Trans 35, 1401-1404.

Wu, H., Sun, L., Zhang, Y., Chen, Y., Shi, B., Li, R., Wang, Y., Liang, J., Fan, D., Wu, G., Wang, D., Li, S., and Shang, Y. (2006). Coordinated regulation of AIB1 transcriptional activity by sumoylation and phosphorylation. J Biol Chem 281, 21848-21856.

Xia, B., Ke, H., and Inouye, M. (2001). Acquirement of cold sensitivity by quadruple deletion of the cspA family and its suppression by PNPase S1 domain in Escherichia coli. Mol Microbiol 40, 179-188.

Xiong, L., Schumaker, K.S., and Zhu, J.K. (2002). Cell signaling during cold, drought, and salt stress. Plant Cell 14 Suppl, S165-183.

Yamanaka, K., Fang, L., and Inouye, M. (1998). The CspA family in Escherichia coli: multiple gene duplication for stress adaptation. Mol Microbiol 27, 247-255. 
Yamanaka, K., and Inouye, M. (2001). Induction of CspA, an E. coli major cold-shock protein, upon nutritional upshift at 37 degrees C. Genes Cells 6, 279-290.

Yamanaka, K., and Inouye, M. (2001). Selective mRNA degradation by polynucleotide phosphorylase in cold shock adaptation in Escherichia coli. J Bacteriol 183, 28082816.

Yang, S.H., Jaffray, E., Hay, R.T., and Sharrocks, A.D. (2003). Dynamic interplay of the SUMO and ERK pathways in regulating Elk-1 transcriptional activity. Mol Cell 12, 63-74.

Yang, S.H., Galanis, A., Witty, J., and Sharrocks, A.D. (2006). An extended consensus motif enhances the specificity of substrate modification by SUMO. Embo J 25, 5083-5093.

Yang, J., Morales, C.R., Medvedev, S., Schultz, R.M., and Hecht, N.B. (2007). In the absence of the mouse DNA/RNA-binding protein MSY2, messenger RNA instability leads to spermatogenic arrest. Biol Reprod 76, 48-54.

Yoo, C.Y., Miura, K., Jin, J.B., Lee, J., Park, H.C., Salt, D.E., Yun, D.J., Bressan, R.A., and Hasegawa, P.M. (2006). SIZ1 small ubiquitin-like modifier E3 ligase facilitates basal thermotolerance in Arabidopsis independent of salicylic acid. Plant Physiol 142, 1548-1558.

Yu, J., Hecht, N.B., and Schultz, R.M. (2001). Expression of MSY2 in mouse oocytes and preimplantation embryos. Biol Reprod 65, 1260-1270.

Yu, J., Deng, M., Medvedev, S., Yang, J., Hecht, N.B., and Schultz, R.M. (2004). Transgenic RNAi-mediated reduction of MSY2 in mouse oocytes results in reduced fertility. Dev Biol 268, 195-206.

Zhang, H., Saitoh, H., and Matunis, M.J. (2002). Enzymes of the SUMO modification pathway localize to filaments of the nuclear pore complex. Mol Cell Biol 22, 6498-6508.

Zhao, J. (2007). Sumoylation regulates diverse biological processes. Cell Mol Life Sci.

Zheng, G., and Yang, Y.C. (2004). ZNF76, a novel transcriptional repressor targeting TATA-binding protein, is modulated by sumoylation. J Biol Chem 279, 4241042421.

Zhong, S., Muller, S., Ronchetti, S., Freemont, P.S., Dejean, A., and Pandolfi, P.P. (2000). Role of SUMO-1-modified PML in nuclear body formation. Blood 95, 2748-2752.

Zhu, J., Dong, C.H., and Zhu, J.K. (2007). Interplay between cold-responsive gene regulation, metabolism and RNA processing during plant cold acclimation. Curr Opin Plant Biol 10, 290-295. 